Simulação numérica do modelo integral KBKZ-PSM com superfície livre

\author{
Juliana Bertoco
}



Data de Depósito:

Assinatura:

\author{
Juliana Bertoco
}

\title{
Solução numérica do modelo constitutivo KBKZ-PSM para escoamentos com superfícies livres
}

Tese apresentada ao Instituto de Ciências Matemáticas e de Computação - ICMC-USP, como parte dos requisitos para obtenção do título de Doutora em Ciências - Ciências de Computação e Matemática Computacional. VERSÃO REVISADA

Área de Concentração: Ciências de Computação e Matemática Computacional

Orientador: Prof. Dr. Murilo Francisco Tomé 
Ficha catalográfica elaborada pela Biblioteca Prof. Achille Bassi e Seção Técnica de Informática, ICMC/USP, com os dados fornecidos pelo(a) autor(a)

Bertoco, Juliana
Solução numérica do modelo constitutivo KBKZ- PSM
para escoamentos com superficies livres / Juliana
Bertoco; orientador Murilo Francisco Tomé. -- São
Carlos, 2016.
$71 \mathrm{p}$.
Tese (Doutorado - Programa de Pós-Graduação em
Ciências de Computação e Matemática Computacional) --
Instituto de Ciências Matemáticas e de Computação,
Universidade de São Paulo, 2016.
1. KBKz- PSM. 2. Diferenças finitas. 3.
Superfícies livres. I. Tomé, Murilo Francisco,
orient. II. Título.




\section{Juliana Bertoco}

\section{Numerical solution of the KBKZ-PSM constitutive model for flows with free surfaces}

Doctoral dissertation submitted to the Instituto de Ciências Matemáticas e de Computação - ICMCUSP, in partial fulfillment of the requirements for the degree of the Doctorate Program in Computer Science and Computational Mathematics. FINAL VERSION

Concentration Area: Computer Science and Computational Mathematics

Advisor: Prof. Dr. Murilo Francisco Tomé 



\section{Agradecimentos}

Primeiramente, agradeço a Deus, meu criador e amigo, pelo dom de minha vida e por Sua presença a iluminar meu caminho ao longo deste trabalho. Aos meus queridos pais, Oscar e Maria Marta, pelas orações, paciência, compreensão e pelo grande apoio ao longo de meus estudos. A minha irmã Joice, ao meu irmão Danilo e aos meus cunhados Mauro e Edivânia por todo carinho, apoio, torcida. E ainda, de maneira especial, aos meus sobrinhos Lara, Heitor e Eloá por todos os momentos de risos e sapequices que me transbordam de alegria.

Ao meu orientador Prof. Dr. Murilo Francisco Tomé por sua disponibilidade, apoio e ensinamentos.

À todos os professores do ICMC-USP, em especial ao professor Dr. Valdemir Garcia Ferreira (in memoriam) por sua orientação durante o meu mestrado.

Ao Prof. Dr. Gilmar Mompean e ao LML-(Laboratoire de Mécanique de Lille) pela oportunidade de pesquisa na Université Lille 1.

Aos amigos do LMACC-USP e a todos os funcionários do ICMC-USP.

À CAPES pelo suporte financeiro ao desenvolvimento deste projeto e ao suporte ao estágio no exterior, processos DS-5633919/D e PDSE-99999.003603/2014-08.

Enfim, a todos que direta ou indiretamente contribuíram para a realização deste trabalho. 


\section{Resumo}

Escoamentos viscoelásticos não estacionários com superfícies livres são comuns em muitos processos industriais e diversas técnicas numéricas têm sido empregadas para reproduzir computacionalmente estes processos. A maioria dos modelos empregados utiliza equações diferenciais na definição do tensor de tensões. Porém, para alguns grupos de fluidos complexos, por exemplo, fluidos de Boger, os modelos integrais mostram-se mais capacitados em fornecer uma boa aproximação para os comportamentos não lineares desses fluidos.

Este trabalho trata da solução numérica do modelo constitutivo integral KBKZ-PSM para escoamentos transientes bidimensionais com superfícies livres. O método numérico proposto é uma técnica numérica que utiliza diferenças finitas para simular escoamentos com superfícies livres na presença de paredes sólidas. As principais características do método numérico proposto são: solução das equações de conservação de quantidade de movimento e massa utilizando um método semi-implícito; a condição de contorno na superfície livre é acoplada à equação de Poisson, o que garante conservação de massa; a discretização do tempo $t^{\prime}$ é realizada por uma nova técnica numérica; o tensor de Finger é calculado pelo método dos campos de deformação e avançado no tempo pelo método de Euler modificado. Essa nova técnica é verificada em escoamentos cisalhantes e elongacionais. Adicionalmente, uma solução analítica desenvolvida para escoamentos em canais bidimensionais é empregada para verificar e analisar a convergência do método proposto. Com relação a escoamentos com superfícies livres, a convergência é verificada por meio de refinamento de malha nas simulações de um jato incidindo sobre placa rígida e no problema do inchamento do extrudado. Finalmente, o método é aplicado para investigar os problemas 'jet buckling' e inchamento do extrudado de fluidos KBKZ-PSM. 
iv

Palavras-chave: Equação constitutiva integral, KBKZ-PSM, Campos de deformação, Diferenças finitas, Superfície livre. 


\section{Abstract}

Unsteady viscoelastic free surface flows are common in many industrial processes and a variety of numerical techniques have been employed to simulate these flows. The majority of constitutive models employed are based on differential equations to define the extra stress tensor. However, for some complex fluids, for instance, Boger fluids, integral models are more adequate to approximate the nonlinear behaviour of these fluids. This work deals with the numerical solution of the integral constitutive model KBKZ-PSM for two-dimensional unsteady free surface flows. The proposed numerical method is a numerical technique that employs finite differences to simulate moving free surface flows that interact with solid walls. The main features of the method are: numerical solution of the momentum and mass equations by an implicit method; the pressure condition on the free surface is implicitly coupled with the Poisson equation for obtaining the pressure field from mass conservation; a novel scheme for defining the past times $t^{\prime}$ is employed; the Finger tensor is calculated by the deformation fields method and is advanced in time by the modified Euler method. This new technique is verified by solving shear and uniaxial elongational flows. Moreover, an analytic solution for channel flows is obtained that is used in the verification and convergence analysis of the proposed methodology. For free surface flows, the assessment of convergence lies on the mesh refinement on the simulation of a jet impinging on a flat surface and the extrudade swell problem. Finally, the new method is applied to investigate the jet buckling phenomenon and extrudate swell of KBKZ-PSM fluids.

Keywords: Integral constitutive equation, KBKZ-PSM, Deformation fields, Finite difference, Free surface 


\section{Lista de Figuras}

3.1 (a) Célula bidimensional. (b) Ilustração da classificação das células. . . . . . . 10

3.2 Representação do fluido. . . . . . . . . . . . . . . . . . . . . 10

3.3 Aproximações para a superfície livre. . . . . . . . . . . . . . . . . . 14

3.4 Discretização do intervalo [0, 20] em 50 e 100 pontos usando a progressão geométrica e a imagem inversa da função memória. . . . . . . . . . . . . . 17

3.5 Interpolação do tensor de Finger $\mathbf{B}_{t^{\prime}\left(t_{n}\right)}\left(\mathbf{x}, t_{n+1}\right)$ em $t^{\prime}\left(t_{n+1}\right) \ldots \ldots \ldots$

3.6 Ilustração das velocidades envolvidas no cálculo da velocidade da partícula $\mathbf{v}\left(\mathbf{x}_{P}\left(t_{n}\right), t_{n+1}\right)=\left(u\left(\mathbf{x}_{P}\left(t_{n}\right), t_{n+1}\right), v\left(\mathbf{x}_{P}\left(t_{n}\right), t_{n+1}\right)\right)$ usando interpolação bilinear. A velocidade $u\left(\mathbf{x}_{P}\left(t_{n}\right), t_{n+1}\right)$ é obtida usando as velocidades representadas pelos símbolos $\boldsymbol{\Delta}$ enquanto $v\left(\mathbf{x}_{P}\left(t_{n}\right), t_{n+1}\right)$ envolve as velocidades exemplificadas em $\boldsymbol{\square} .20$

4.1 Erros relativos no cálculo de $\tau^{x x}$ em $t=10 \lambda$ como função de $N$ usando $\dot{\gamma}=1 \mathrm{~s}^{-1}$ e $\dot{\varepsilon}=0.3 \mathrm{~s}^{-1}$ para escoamentos cisalhantes (a) e elongacionais (b). . . . . . . 25

4.2 Erros no cálculo de $\tau^{x x}$ para $0.1 \leq \dot{\varepsilon} \leq 0.49, \lambda=1 \mathrm{~s}, a=100 \mathrm{~Pa}$ usando a imagem inversa da função memória (círculos) e a progressão geométrica (triângulos) para $N=30,50$.

4.3 Solução numérica em um canal usando fluido KBKZ com 1 módulo de relaxamento. Comparação de $\partial u / \partial y /(L / U)(\mathrm{a}), \tau^{x x}(y) /\left(\rho_{0} U^{2}\right)(\mathrm{b})$ e $\tau^{y y}(y) /\left(\rho_{0} U^{2}\right)$ (c) obtidas nas malhas $I, I I, I I I, I V$ em comparação com a respectiva solução analítica 
4.4 Solução numérica para o Fluido $S 1$ usando as malhas I, II e III. (a) $u(y) / U$; (b) $\tau^{x x}(y) /\left(\rho_{0} U^{2}\right) ;\left(\right.$ c) $\tau^{x y}(y) /\left(\rho_{0} U^{2}\right) ;(\mathrm{d}) \tau^{y y}(y) /\left(\rho_{0} U^{2}\right)$. A curva de cor laranja em (a) representa o perfil de velocidades Newtoniano.

4.5 Perfil da velocidade $u$ em seções transversais do canal no tempo $t=100 \mathrm{~s}$. Os resultados são apresentados para algumas posições de $x / L$.

4.6 (a) Exemplo do problema Jet-Buckling para um fluido Newtoniano [61]; (b) Descrição do domínio usado na simulação do jato incidente sobre uma parede rígida. 34

4.7 Simulação do jato incidindo sobre uma superfície rígida. Visualização do fluido nas malhas M1, M2, M3, M4, M5 e M6.

4.8 Simulação numérica do jato incidente sobre placa rígida utilizando fluido Newtoniano e fluido $B$ com $U=0.2 \mathrm{~ms}^{-1}$. Visualização do escoamento em alguns tempos.

4.9 Simulação numérica do jato incidente sobre uma placa rígida utilizando fluido Newtoniano e fluido $B \operatorname{com} U=0.4 \mathrm{~ms}^{-1}$. Visualização do escoamento em alguns tempos.

4.10 Simulação numérica do jato incidente sobre uma placa rígida utilizando fluido Newtoniano e fluido $B \operatorname{com} U=0.6 \mathrm{~ms}^{-1}$. Visualização do escoamento em alguns tempos.

4.11 Simulação numérica do jato incidente sobre uma placa rígida utilizando fluido Newtoniano e fluido $S 1$ com $U=0.28 \mathrm{~ms}^{-1}$. Visualização do escoamento em alguns tempos.

4.12 Resultado experimental (a) e ilustração do domínio utilizado no problema do inchamento do extrudado $(\mathrm{b}) . \ldots \ldots \ldots \ldots$

4.13 Solução numérica no tempo $t=15 \mathrm{~s}$. Superfície do fluido nas malhas $M_{I}, M_{I I}$, $M_{I I I}, M_{I V}$ e $M_{V}$.

4.14 Perfil da solução das velocidades nas direções $x, y$ e da pressão para $\dot{\gamma}_{a}=16$ no tempo $t=15 \mathrm{~s}$.

4.15 Perfil da solução para as componentes do tensor $\tau$ com $\dot{\gamma}_{a}=16$ no tempo $t=15 \mathrm{~s} . \quad 46$

4.16 Visualização da solução numérica nos tempos $t=1.5 \mathrm{~s}$ e $t=2.5 \mathrm{~s}$ considerando

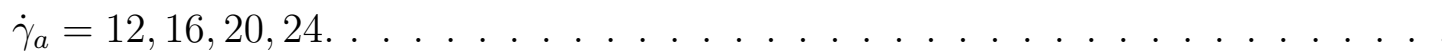


4.17 Visualização da solução numérica nos tempos $t=3.5 \mathrm{~s}$ e $t=4.5 \mathrm{~s}$ considerando $\dot{\gamma}_{a}=12,16,20,24 \ldots \ldots \ldots \ldots \ldots \ldots \ldots$

4.18 Visualização da solução numérica nos tempos $t=6.0 \mathrm{~s}$ e $t=15.0$ s considerando

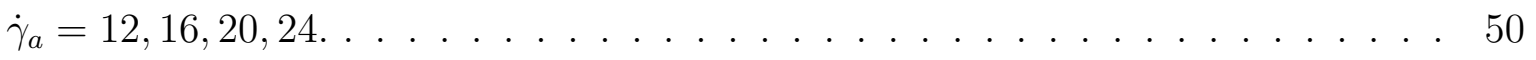

4.19 Perfil da superfície livre para alguns valores de $\dot{\gamma}_{a} \ldots \ldots \ldots$. . . . . . . . . 51

4.20 Valores de de $\chi$ (a) e $S_{r}$ (b) $\operatorname{com} \theta=0$ em comparação como os resultados obtidos por Mitsoulis [17] para $\theta=-0.15 \ldots \ldots \ldots \ldots$. . . . . . . 53

4.21 (a)Solução numérica para $\dot{\gamma}_{a}=21$ utilizando $R e^{*}$; (b) inchamento $\chi$ utilizando $R e$ e $R e^{*}$ em comparação com a solução de Mitsoulis [17] . . . . . . . . . . . . 53

A.1 Definição do canal utilizado no cálculo da solução analítica. . . . . . . . . . . . 67 


\section{Lista de Tabelas}

4.1 Erros relativos para o cálculo de $\tau^{x x}$ considerando escoamentos elongacionais e cisalhantes em função de $N$.

4.2 Erros relativos de $\frac{\partial u(y)}{\partial y}, \tau^{x x}, \tau^{y y}$, obtidos nas malhas $I, I I, I I I, I V$. A última coluna mostra o valor médio para $p_{x} \approx\left(\sum_{j=1}^{J_{l}} \frac{p_{i_{\text {out }}, j}-p_{i_{\text {out }-1, j}}}{\delta x}\right) / J_{l}$, onde $i_{\text {out }}$ denota o índice da célula mais próxima à saída do canal.

4.3 Ordens de convergência obtidas nas malhas $I, I I, I I I, I V$ usando os dados apresentados na Tabela $4.2 \ldots \ldots \ldots$. . . . . . . . . . . . . . . . . . . 29

4.4 Definição do fluido empregado na simulação do escoamento em um canal (obtidos de Quinzani [54]) . . . . . . . . . . . . . . . . . . . . . 31

4.5 Parâmetros do Fluido $B$ usados no problema do jato incidente sobre placa paralela. (ver Mitsoulis $[17]) \ldots \ldots \ldots \ldots$

4.6 Dados usados no problema do jato incidente sobre uma placa paralela. . . . . . 37

4.7 Parâmetros do Fluido M1 utilizado no problema do inchamento do extrudado. (ver Mitsoulis [17]) . . . . . . . . . . . . . . . . . . 43

4.8 Dados utilizados na simulação do inchamento do extrudado . . . . . . . . . . . 47

4.9 Valores de $S_{r}$ e $\chi$ em comparação com a solução de Mitsoulis [17] . . . . . . . . 52

4.10 Valores de $S_{r}$ e $\chi$ considerando $R e$ e $R e^{*}$ em comparação com a solução de

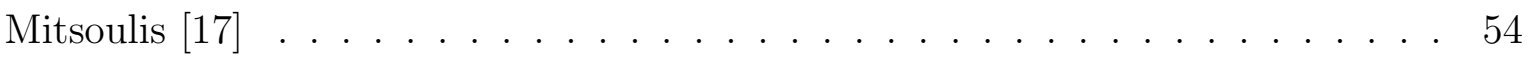




\section{Sumário}

1 Introdução $\quad 1$

2 Formulação Matemática $\quad 5$

2.1 Equações Governantes . . . . . . . . . . . . . . . . . 5

3 Método Numérico $\quad 9$

3.1 Cálculo de $\mathbf{v}\left(\mathbf{x}, t_{n+1}\right)$ e $p\left(\mathbf{x}, t_{n+1}\right) \ldots \ldots \ldots \ldots \ldots \ldots$

3.2 Cálculo do tensor de tensões extra $\boldsymbol{\tau}\left(\mathbf{x}, t_{n+1}\right) \ldots \ldots \ldots$

4 Resultados numéricos $\quad 23$

4.1 Verificação da nova distribuição dos pontos $t_{j}^{\prime} \ldots \ldots$. . . . . . . . . 23

4.2 Escoamentos confinados . . . . . . . . . . . . . . . . . . . . 28

4.2.1 Verificação em um canal para fluido KBKZ-PSM com 1 módulo de relaxamento . . . . . . . . . . . . . . . . . . . 28

4.2.2 Verificação em um canal para fluido KBKZ-PSM multi-módulos ..... 31

4.3 Escoamentos com superfícies livres . . . . . . . . . . . . . . . 33

4.3.1 Simulação do Jet-Buckling . . . . . . . . . . . . . . . . . 33

4.3.2 Simulação do Extrudate Swell . . . . . . . . . . . . . . . . . . 42

5 Conclusões $\quad 55$

A Solução analítica do escoamento em canal para um fluido KBKZ com 1-módulo 
de relaxamento 


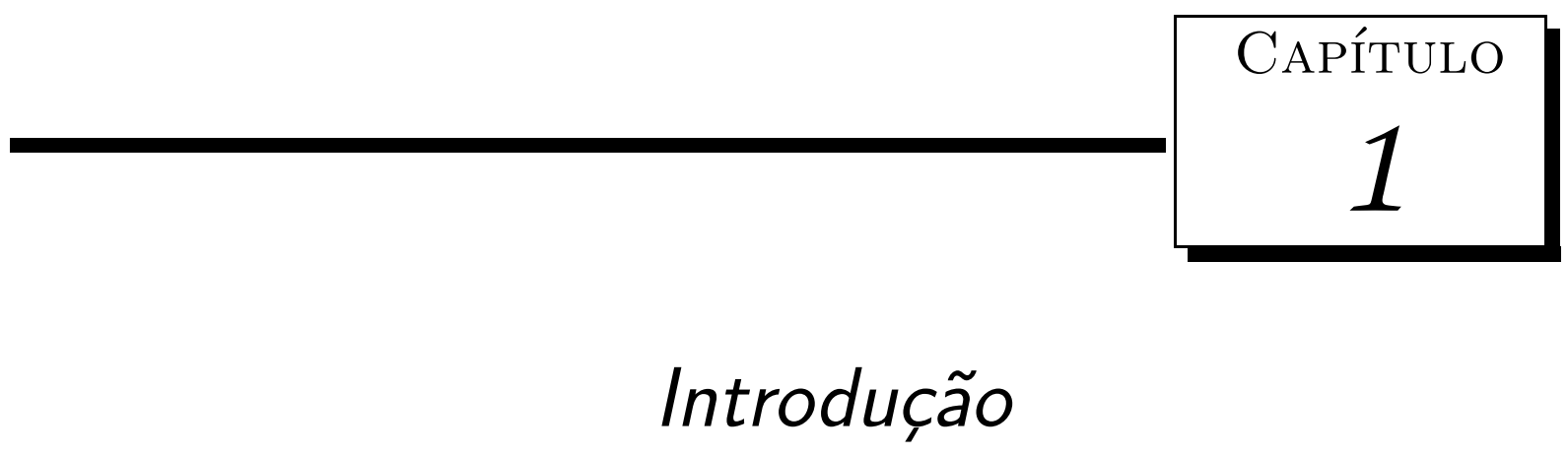

Escoamentos viscoelásticos com superfície livre são importantes em muitos processos industriais, por exemplo, em moldagem por injeção e extrusão de perfis. Nessas aplicações, o escoamento pode possuir várias superfícies livres móveis e envolver fluidos não Newtonianos o que torna esses problemas altamente complexos. Muitos pesquisadores têm trabalhado no desenvolvimento de métodos numéricos para simulação de escoamentos viscoelásticos com superfície livre porém a aplicação rigorosa das condições de tensão na superfície livre é um problema que ainda não foi totalmente resolvido. Na maioria dos trabalhos com superfície livre, as equações constitutivas são aproximadas por equações diferenciais, como por exemplo, os modelos UCM [1-3], Oldroyd-B [4-7], Phan-Thien-Tanner [8-10], FENE-CR (FENE-P) [11], eXtended Pom-Pom [12,13], entre outros. No entanto, os avanços em recursos computacionais têm motivado pesquisadores a considerar modelos reológicos mais sofisticados que empregam equações integrais ao invés de equações diferenciais parciais. Uma das razões é que modelos constitutivos integrais são reconhecidos por aproximar de maneira correta a reologia de polímeros, como por exemplo HDPE [14,15], LDPE [16] e fluidos do tipo Boger [17, 18].

Entre os modelos integrais investigados, a equação constitutiva KBKZ-PSM [19, 20] têm sido considerada por muitos pesquisadores que estudam métodos numéricos para esta classe de fluidos. O modelo KBKZ foi originalmente desenvolvido usando as ideias da teoria da 
elasticidade de Rubber (ver [20]). Em sua forma original, este modelo consiste em uma classe de equações constitutivas porque admite uma grande variedade de funções para a energia livre. Nesse modelo, o líquido viscoelástico é considerado como uma borracha relaxante na qual tanto a energia elástica quanto a tensão podem relaxar. Um dos métodos para se chegar a um modelo específico KBKZ consiste em ajustar os dados da tensão. A resposta do modelo é muitas vezes deficiente, se duas tensões súbitas são de sinais opostos [21], mas esta é uma deformação severa e é pouco provável que o fluido sofra este tipo de deformação. Na ausência de tais deformações, o desempenho do modelo é significativamente bom e o modelo pode ser caracterizado por meio de dados à partir de medições da viscosidade e da primeira diferença de tensões normais do fluxo em cisalhamento constante. Se mais de um módulo são necessários para ajustar o espectro de relaxação, os dados de pequenas oscilações da tensão fornecem os coeficientes da função memória. Os escoamento testados neste trabalho não apresentam deformações severas na tensão de cisalhamento. Para alguns polímeros, como polietilenos de baixa densidade, a dependência da tensão nas equações KBKZ é dividida em dependência temporal e de deformação e o comportamento não linear pode ser capturado por uma função de decaimento, para a qual muitas formas foram propostas (ver [22]), incluindo a função PSM (Papanastasiou-Scriven-Macosko [23]) que será usada neste trabalho. Aproximações do modelo KBKZ-PSM com dados experimentais em fluxos elongacionais e cisalhantes para polietilenos de baixa e alta densidade estacionários e transientes são apresentados por Mitsoulis [24].

Uma detalhada discussão sobre a importância do modelo constitutivo integral KBKZ e o desenvolvimento das técnicas numéricas para aproximar modelos integrais pode ser encontrada nos trabalhos de Tanner [25] e Mitsoulis [24].

A grande maioria dos problemas investigados utilizando o modelo integral KBKZ-PSM envolvem escoamentos confinados, como por exemplo fluxos em canais [26,27] e fluxos em contrações abruptas [16,28-30]. Escoamentos com superfície livre também foram investigados. O problema clássico que envolve superfície livre, conhecido como inchamento do extrudado, foi apresentado em $[14,17,18,31-34]$. Entretanto, muitas dessas simulações empregam a discretização em elementos finitos em estado estacionário (ver [35-38]). Um trabalho envolvendo fluxos com superfície livre transiente em modelos integrais foi apresentado por McKinley e Sridhar [39] e também outros escoamentos foram apresentados em [40-42].

Embora o modelo KBKZ tenha sido aplicado para resolver uma grande variedade de escoa- 
mentos viscoelásticos, para problemas nos quais a superfície livre interage com paredes sólidas, tão comuns em aplicações industriais, como por exemplo, enchimento de recipientes, a metodologia ainda não havia sido aplicada. Neste trabalho, apresenta-se um método numérico bidimensional para simular escoamentos viscoelásticos utilizando o modelo integral KBKZ-PSM [23] capaz de simular escoamentos com superfícies livres móveis que interagem com paredes rígidas. A metodologia apresentada é capaz de simular o efeito de dobras (Buckling) que pode ocorrer quando um jato interage com uma parede sólida. A técnica apresentada aqui também será utilizada para escoamentos com fluidos complexos conhecidos como fluidos de Boger. Esse tipo de fluido foi inicialmente descrito com o modelo constitutivo diferencial Oldroyd-B (ver [17]); e os resultados foram bons apenas em regiões de baixa tensão de cisalhamento. Em regiões de alta tensão de cisalhamento, estes fluidos tendem a assumir um comportamento não linear, e modelos integrais mostraram-se capazes de aproximar bem os resultados experimentais (ver $[17,18,43])$. Nas simulações apresentadas neste trabalho para o problema do inchamento do extrudado, o fluido do tipo Boger denominado Fluido $M 1$ foi utilizado e simulações em altas taxas de cisalhamento com elevados valores de inchamento do extrudado foram obtidos, demostrando que a metodologia apresentada aqui é capazes de simular esta classe de fluidos complexos. 
CAPÍTULO

\section{Formulação Matemática}

Neste capítulo apresentam-se as equações que governam escoamentos viscoelásticos incompressíveis e isotérmicos governados pelo modelo integral KBKZ.

\subsection{Equações Governantes}

Na forma adimensional, as equações de conservação de massa e de quantidade de movimento, são respectivamente, escritas (ver [30]) como :

$$
\begin{aligned}
\nabla \cdot \mathbf{v} & =0 \\
\frac{\partial \mathbf{v}}{\partial t}+\nabla \cdot(\mathbf{v v}) & =-\nabla p+\frac{1}{R e} \nabla^{2} \mathbf{v}+\nabla \cdot \mathbf{\Phi}+\frac{1}{F r^{2}} \mathbf{g}
\end{aligned}
$$

Na equação (2.2), $\boldsymbol{\Phi}$ é um tensor não Newtoniano que é derivado do tensor de tensões extra $\tau$ pela seguinte equação:

$$
\boldsymbol{\Phi}=\boldsymbol{\tau}-\frac{1}{R e} \dot{\gamma}, \text { onde } \dot{\gamma}=\nabla \mathbf{v}+(\nabla \mathbf{v})^{\mathrm{T}}
$$

Para completar este sistema de equações, precisa-se considerar um modelo reológico que estabeleça o comportamento do escoamento. Neste trabalho, considera-se o modelo constitutivo 
integral KBKZ-PSM [23]. Neste modelo, o tensor de tensões extra é definido por

$$
\boldsymbol{\tau}(\mathbf{x}, t)=\int_{-\infty}^{t} M\left(t-t^{\prime}\right) H\left(I_{1}, I_{2}\right) \mathbf{B}_{t^{\prime}}(\mathbf{x}, t) d t^{\prime}
$$

onde

$$
M\left(t-t^{\prime}\right)=\sum_{k=1}^{m_{1}} \frac{a_{k}}{\lambda_{k} W i} e^{-\frac{t-t^{\prime}}{\lambda_{k} W i}}
$$

é a função memória, $\lambda_{k}, a_{k}, m_{1}$ são os tempos de relaxamento, os módulos destes relaxamentos e a quantidade destes módulos, respectivamente. A equação

$$
H\left(I_{1}, I_{2}\right)=\frac{\alpha}{\alpha-3+\beta I_{1}+(1-\beta) I_{2}}
$$

é a função de decaimento de Papanastasiou-Scriven-Macosko [23] e $\mathbf{B}_{t^{\prime}}(\mathbf{x}, t)$ é o tensor de Finger. Nessa função, $I_{1}=\operatorname{tr}\left[\mathbf{B}_{t^{\prime}}(\mathbf{x}, t)\right]$ e $I_{2}=\frac{1}{2}\left(\left(I_{1}\right)^{2}-\operatorname{tr}\left[\mathbf{B}_{t^{\prime}}^{2}(\mathbf{x}, t)\right]\right)$, são o primeiro e o segundo invariantes de $\mathbf{B}_{t^{\prime}}(\mathbf{x}, t)$, respectivamente. Os parâmetros $a_{k}, \lambda_{k}, \alpha, \beta$ são definidos ajustando as propriedades reológicas específicas de cada fluido.

Nesse trabalho, adota-se o método de campos de deformação [45] para o tratamento do tensor de Finger. Nessa metodologia, definem-se $N+1$ tempos $t_{j}^{\prime}$, discretizados no intervalo $[0, t]$, nos quais são armazenados o histórico de deformação. Esse histórico é calculado através da seguinte equação de convecção:

$$
\frac{\partial}{\partial t} \mathbf{B}_{t_{j}^{\prime}}(\mathbf{x}, t)+\mathbf{v}(\mathbf{x}, t) \cdot \nabla \mathbf{B}_{t_{j}^{\prime}}(\mathbf{x}, t)=[\nabla \mathbf{v}(\mathbf{x}, t)]^{T} \cdot \mathbf{B}_{t_{j}^{\prime}}(\mathbf{x}, t)+\mathbf{B}_{t_{j}^{\prime}}(\mathbf{x}, t) \cdot \nabla \mathbf{v}(\mathbf{x}, t)
$$

Nessas equações $R e=\frac{\rho_{0} U L}{\eta_{0}}$ é o numero de Reynolds, $F r=\frac{U}{\sqrt{L g}}$ é o numero de Froude e $W i=\lambda_{r e f} \frac{U}{L}$ é o número de Weissenberg; $U$ é a escala de velocidade e $L$ é o tamanho característicos, $g$ e $\rho_{0}$ são a aceleração da gravidade e a densidade do fluido respectivamente. A viscosidade é definida como $\eta_{0}=\sum_{k=1}^{m_{1}} a_{k} \lambda_{k}$ e $\lambda_{r e f}=\sum_{k=1}^{m_{1}} \frac{a_{k} \lambda_{k}^{2}}{a_{k} \lambda_{k}}$ é o tempo médio de relaxamento [17].

A adimensionalização das equações foi feita como segue, onde as variáveis dimensionais são sobrescritas por uma barra. 


$$
\overline{\mathbf{x}}=L \mathbf{x}, \bar{t}=\frac{L}{U} t, \overline{\mathbf{v}}=U \mathbf{v}, \quad \bar{p}=\rho_{0} U^{2} p, \quad \overline{\boldsymbol{\tau}}=\rho_{0} U^{2} \boldsymbol{\tau}, \quad \bar{\lambda}_{k}=\lambda_{r e f} \lambda_{k}, \quad \bar{a}_{k}=\rho_{0} U^{2} a_{k}
$$

As equações (2.1)-(2.7) são resolvidas em um sistema cartesiano bidimensional onde $p=$ $p(\mathbf{x}, t), \mathbf{v}=(u(\mathbf{x}, t), v(\mathbf{x}, t))^{T} \mathrm{e}$

$$
\begin{gathered}
\boldsymbol{\tau}(\mathbf{x}, t)=\left[\begin{array}{cc}
\tau^{x x} & \tau^{x y} \\
\tau^{x y} & \tau^{y y}
\end{array}\right], \quad \boldsymbol{\Phi}(\mathbf{x}, t)=\left[\begin{array}{cc}
\Phi^{x x} & \Phi^{x y} \\
\Phi^{x y} & \Phi^{y y}
\end{array}\right], \\
\nabla \mathbf{v}(\mathbf{x}, t)=\left[\begin{array}{cc}
\frac{\partial u}{\partial x} & \frac{\partial v}{\partial x} \\
\frac{\partial u}{\partial y} & \frac{\partial v}{\partial y}
\end{array}\right], \quad \mathbf{B}_{t^{\prime}}(\mathbf{x}, t)=\left[\begin{array}{ccc}
B_{t^{\prime}}^{x x} & B_{t^{\prime}}^{x y} & 0 \\
B_{t^{\prime}}^{x y} & B_{t^{\prime}}^{y y} & 0 \\
0 & 0 & 1
\end{array}\right] .
\end{gathered}
$$

Note que, para calcular o tensor total em 2D, consideramos o tensor de Finger tridimensional. Esta definição leva em consideração a ausência de deformação na terceira coordenada $\left(B_{t^{\prime}}^{z z}\right.$ em escoamentos tridimensionais), além de garantir a propriedade de determinante diferente de zero para o tensor de Finger.

Considerando escoamentos cartesianos bidimensionais as equações governantes (2.1)-(2.3) são discretizadas como (ver [30])

$$
\begin{aligned}
\frac{\partial u}{\partial x}+\frac{\partial v}{\partial y} & =0 \\
\frac{\partial u}{\partial t}+\frac{\partial\left(u^{2}\right)}{\partial x}+\frac{\partial(u v)}{\partial y} & =-\frac{\partial p}{\partial x}+\frac{1}{R e}\left[\frac{\partial^{2} u}{\partial x^{2}}+\frac{\partial^{2} u}{\partial y^{2}}\right]+\frac{\partial \Phi^{x x}}{\partial x}+\frac{\partial \Phi^{x y}}{\partial y}+\frac{1}{F r^{2}} g_{x} \\
\frac{\partial v}{\partial t}+\frac{\partial(u v)}{\partial x}+\frac{\partial\left(v^{2}\right)}{\partial y} & =-\frac{\partial p}{\partial y}+\frac{1}{R e}\left[\frac{\partial^{2} v}{\partial x^{2}}+\frac{\partial^{2} v}{\partial y^{2}}\right]+\frac{\partial \Phi^{x y}}{\partial x}+\frac{\partial \Phi^{y y}}{\partial y}+\frac{1}{F r^{2}} g_{y}
\end{aligned}
$$

nas quais (ver equação (2.3))

$$
\begin{aligned}
\Phi^{x x} & =\tau^{x x}-\frac{2}{R e}\left(\frac{\partial u}{\partial x}\right) \\
\Phi^{x y} & =\tau^{x y}-\frac{1}{R e}\left(\frac{\partial u}{\partial y}+\frac{\partial v}{\partial x}\right) \\
\Phi^{y y} & =\tau^{y y}-\frac{2}{R e}\left(\frac{\partial v}{\partial y}\right)
\end{aligned}
$$


O tensor $\boldsymbol{\tau}$ (ver (2.4)) é escrito como

$$
\begin{aligned}
\tau^{x x} & =\int_{-\infty}^{t} \sum_{k=1}^{m_{1}} \frac{a_{k}}{W i \lambda_{k}} e^{-\frac{t-t^{\prime}}{W i \lambda_{k}}} \frac{\alpha}{\alpha-3+\beta I_{1}+(1-\beta) I_{2}} B_{t^{\prime}}^{x x} d t^{\prime}, \\
\tau^{x y} & =\int_{-\infty}^{t} \sum_{k=1}^{m_{1}} \frac{a_{k}}{W i \lambda_{k}} e^{-\frac{t-t^{\prime}}{W i \lambda_{k}}} \frac{\alpha}{\alpha-3+\beta I_{1}+(1-\beta) I_{2}} B_{t^{\prime}}^{x y} d t^{\prime}, \\
\tau^{y y} & =\int_{-\infty}^{t} \sum_{k=1}^{m_{1}} \frac{a_{k}}{W i \lambda_{k}} e^{-\frac{t-t^{\prime}}{W i \lambda_{k}}} \frac{\alpha}{\alpha-3+\beta I_{1}+(1-\beta) I_{2}} B_{t^{\prime}}^{y y} d t^{\prime} .
\end{aligned}
$$

As componentes do tensor de Finger são calculadas pelas equações (ver (2.7))

$$
\begin{aligned}
\frac{\partial}{\partial t} B_{t^{\prime}}^{x x} & =-\frac{\partial}{\partial x}\left(u B_{t^{\prime}}^{x x}\right)-\frac{\partial}{\partial y}\left(v B_{t^{\prime}}^{x x}\right)+2\left[\frac{\partial u}{\partial x} B_{t^{\prime}}^{x x}+\frac{\partial u}{\partial y} B_{t^{\prime}}^{x y}\right] \\
\frac{\partial}{\partial t} B_{t^{\prime}}^{x y} & =-\frac{\partial}{\partial x}\left(u B_{t^{\prime}}^{x y}\right)-\frac{\partial}{\partial y}\left(v B_{t^{\prime}}^{x y}\right)+\frac{\partial u}{\partial y} B_{t^{\prime}}^{y y}+\frac{\partial v}{\partial x} B_{t^{\prime}}^{x x} \\
\frac{\partial}{\partial t} B_{t^{\prime}}^{y y} & =-\frac{\partial}{\partial x}\left(u B_{t^{\prime}}^{y y}\right)-\frac{\partial}{\partial y}\left(v B_{t^{\prime}}^{y y}\right)+2\left[\frac{\partial v}{\partial x} B_{t^{\prime}}^{x y}+\frac{\partial v}{\partial y} B_{t^{\prime}}^{y y}\right] .
\end{aligned}
$$




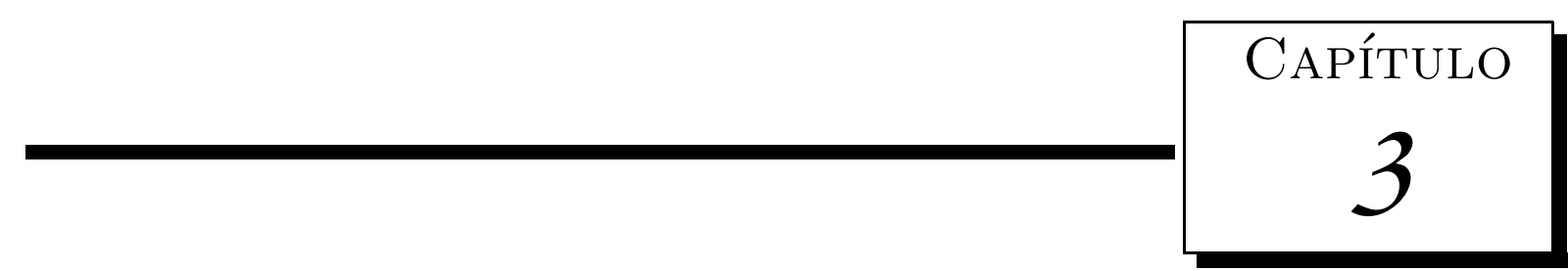

\section{Método Numérico}

As equações governantes são resolvidas por uma variante do método das partículas marcadoras $[46,47]$ que emprega o método de diferenças finitas em uma malha deslocada. As componentes da velocidade são definidas no meio das faces das células, enquanto as outras variáveis, denotas por $\boldsymbol{\Psi}$, são definidas no centro das células computacionais (veja Fig. 3.1a). $\mathrm{Na}$ técnica adotada neste trabalho, consideram-se escoamentos que possuem superfície livre; a qual é definida por partículas marcadoras que se movimentam com a velocidade local do fluido (ver Tomé e colaboradores [48,49]). A visualização da superfície livre é feita unindo-se as partículas marcadoras por retas (ver Fig. 3.2a). O corpo do fluido é representado pela região contida pela curva fechada definida pela união das partículas (ver Fig. 3.2b). Nessa estratégia, as células computacionais da malha são agrupadas em tipos de células, definidas (ver [49], Fig. 3.1b) como segue:

- Entrada de fluidos (Inflow - (I)): Células que simulam entrada de fluido.

- Saída de fluidos (Outflow - (O)): Células que simulam regiões de saída de fluido.

- Contornos (Boundary - (B)): Células que definem contornos rígidos.

- Células vazias (Empty - (E)): Células que não contém fluido ( ver células brancas na Fig. $3.1 \mathrm{~b})$; 
- Superfície livre (Surface - $(\mathbf{S})$ ): Células que contém fluido e tem uma ou mais faces em contato com células vazias.

- Células cheias (Full - $(\mathbf{F})$ ): Células que definem a região de fluido. Estas células não possuem nenhuma de suas faces em contato com células vazias.

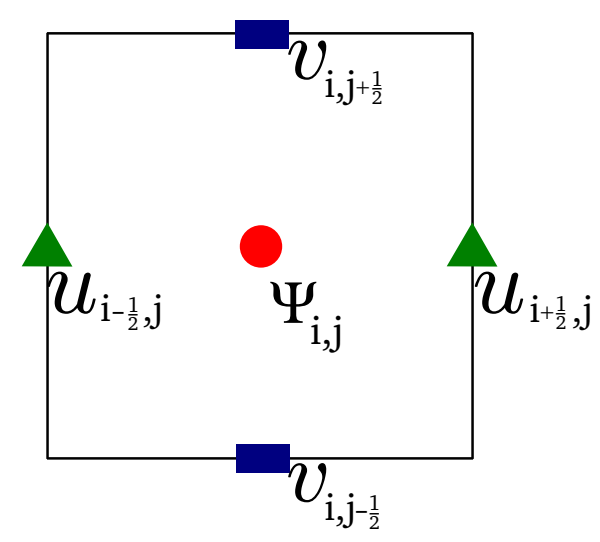

(a)

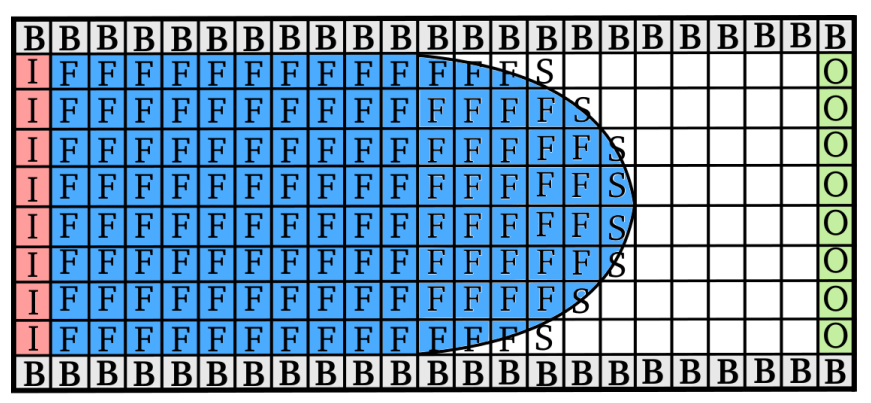

(b)

Figura 3.1: (a) Célula bidimensional. (b) Ilustração da classificação das células.

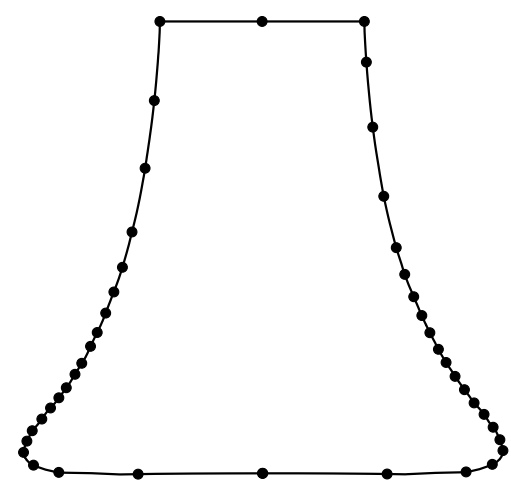

(a) Definição da superfície do fluido.

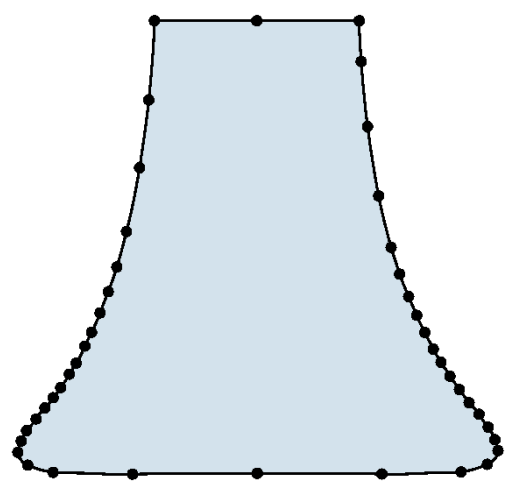

(b) Visualização do volume do fluido.

Figura 3.2: Representação do fluido.

Para resolver as equações (2.1)-(2.3), deve-se especificar condições de contorno para o campo de velocidades. Em células de entrada de fluido (inflows) um campo de velocidades $\left(\mathbf{V}_{\mathbf{i n f}}\right)$ é 
prescrito e em saídas de fluidos (outflows) assume-se um escoamento completamente desenvolvido e as condições homogêneas de Neumann $\frac{\partial \mathbf{v}}{\partial n}=\mathbf{0}$ são adotadas, onde $n$ é a direção normal em relação ao contorno. Na superfície livre é necessário aplicar condições de contorno especiais para os campos de velocidades e de pressão. Neste trabalho, considera-se que a superfície livre se move em uma atmosfera passiva. Na ausência de forças de tensão superficial, as componentes normal e tangencial da tensão devem ser contínuas, de modo que em superfícies livres devem ser satisfeitas as condições (ver [50]):

$$
\mathbf{n} \cdot \boldsymbol{\sigma} \cdot \mathbf{n}^{\mathrm{T}}=0 \quad \text { e } \quad \mathbf{m} \cdot \boldsymbol{\sigma} \cdot \mathbf{n}^{\mathrm{T}}=0
$$

onde $\mathbf{n}$ é o vetor normal à superfície livre e $\mathbf{m}$ é o vetor tangencial.

O tensor de tensões total , $\boldsymbol{\sigma}$ é dado por

$$
\boldsymbol{\sigma}=-p \mathbf{I}+\frac{1}{R e} \dot{\gamma}+\boldsymbol{\Phi}
$$

onde $\boldsymbol{\Phi}$ e $\dot{\gamma}$ são os tensores obtidos da transformação EVSS (2.3). Considerando escoamentos bidimensionais, os vetores unitários $\mathbf{n}$ e $\mathbf{m}$ na equação $(3.1)$ podem ser escrito como $\mathbf{n}=\left(n_{x}, n_{y}\right)$ e $\mathbf{m}=\left(n_{y},-n_{x}\right)$, e estas condições podem ser escritas como

$$
p=\frac{2}{R e}\left[\frac{\partial u}{\partial x} n_{x}^{2}+\left(\frac{\partial u}{\partial y}+\frac{\partial v}{\partial x}\right) n_{x} n_{y}+\frac{\partial v}{\partial y} n_{y}^{2}\right]+\Phi^{x x} n_{x}^{2}+2 \Phi^{x y} n_{x} n_{y}+\Phi^{y y} n_{y}^{2}
$$

e

$$
\frac{1}{R e}\left[2\left(\frac{\partial u}{\partial x}-\frac{\partial v}{\partial y}\right) n_{x} n_{y}+\left(\frac{\partial u}{\partial y}+\frac{\partial v}{\partial x}\right)\left(n_{y}^{2}-n_{x}^{2}\right)\right]+\left(\Phi^{x x}-\Phi^{y y}\right) n_{x} n_{y}+\Phi^{x y}\left(n_{y}^{2}-n_{x}^{2}\right)=0
$$

\section{Algoritmo computacional}

As soluções $\mathbf{v}\left(\mathbf{x}, t_{n+1}\right), p\left(\mathbf{x}, t_{n+1}\right)$ e $\boldsymbol{\tau}\left(\mathbf{x}, t_{n+1}\right)$ no tempo $t_{n+1}=t_{n}+\delta t$ são obtidas em duas partes: primeiramente, usando $\boldsymbol{\tau}\left(\mathbf{x}, t_{n}\right)$, os campos de velocidade e pressão são calculados no tempo $t_{n+1}$. No segundo passo, $\mathbf{v}\left(\mathbf{x}, t_{n+1}\right)$ é usado para calcular o tensor $\boldsymbol{\tau}\left(\mathbf{x}, t_{n+1}\right)$ pelo método dos campos de deformação ( ver [44] e [45]), como segue. 


\subsection{Cálculo de $\mathbf{v}\left(\mathrm{x}, t_{n+1}\right)$ e $p\left(\mathrm{x}, t_{n+1}\right)$}

O método numérico utilizado para resolver o sistema de equações (2.1)-(2.2) é baseado no algoritmo implícito proposto por Oishi e colaboradores [13] para resolver escoamentos viscoelásticos com superfície livre, usando o modelo XPP (eXtended Pom-Pom). Assume-se que no tempo $t_{n+1}$ as variáveis $\mathbf{v}\left(\mathbf{x}, t_{n}\right)=\mathbf{v}^{(n)}, p\left(\mathbf{x}, t_{n}\right)=p^{(n)}, \boldsymbol{\tau}\left(\mathbf{x}, t_{n}\right)=\boldsymbol{\tau}^{(n)}$ e as posições das partículas marcadoras $\mathbf{x}\left(t_{n}\right)=\mathbf{x}^{(n)}$, são conhecidas. As soluções em $t_{n+1}$ para $\mathbf{v}\left(\mathbf{x}, t_{n+1}\right)$, $p\left(\mathbf{x}, t_{n+1}\right)$ e $\mathbf{x}\left(t_{n+1}\right)$ são obtidas como segue:

P1. Calcula-se $\dot{\gamma}^{(n)}=\left[\nabla \mathbf{v}^{(n)}+\left(\nabla \mathbf{v}^{(n)}\right)^{\mathrm{T}}\right]$ e usando (2.3) obtém-se $\boldsymbol{\Phi}^{(n)}=\boldsymbol{\tau}^{(n)}-\frac{1}{R e} \dot{\gamma}^{(n)}$.

P2. Calcula-se o campo de velocidades:

A equação (2.2) é discretizada pelo método de Euler semi-implícito, assim

$$
\frac{\mathbf{v}^{(n+1)}-\mathbf{v}^{(n)}}{\delta t}+\operatorname{conv}(\mathbf{v v})^{(n)}=\frac{1}{R e} \nabla^{2} \mathbf{v}^{(n+1)}-\nabla p^{(n+1)}+\nabla \cdot \boldsymbol{\Phi}^{(n)}+\frac{1}{F r^{2}} \mathbf{g}^{(n)}
$$

onde $\operatorname{conv}(\mathbf{v v})^{(n)}$ é uma aproximação explícita para $\nabla \cdot(\mathbf{v v})$ em $t_{n}$ que é calculada pelo método de alta ordem CUBISTA [51]. Um campo de velocidades intermediário $\widetilde{\mathbf{v}}^{(n+1)}$ é utilizado para desacoplar as equações de conservação de massa e de movimento, seguindo as ideias do método de projeção (ver [13]). Assim, a equação (3.5) é reescrita como

$$
\frac{\widetilde{\mathbf{v}}^{(n+1)}}{\delta t}-\frac{1}{R e} \nabla^{2} \widetilde{\mathbf{v}}^{(n+1)}=\frac{\mathbf{v}^{(n)}}{\delta t}-\operatorname{conv}(\mathbf{v v})^{(n)}-\nabla p^{(n)}+\nabla \cdot \boldsymbol{\Phi}^{(n)}+\frac{1}{F r^{2}} \mathbf{g}^{(n)},
$$

na qual $p^{(n)}$ é uma aproximação para $\widetilde{p}^{(n+1)}$ e $\mathbf{v}^{(n)}$ é uma aproximação para $\widetilde{\mathbf{v}}^{(n)}$. Sabe-se que $\widetilde{\mathbf{v}}^{(n+1)}$ pode não satisfazer a conservação de massa [52]. Entretanto, existe uma função potencial $\psi^{(n+1)}$ tal que

$$
\mathbf{v}^{(n+1)}=\widetilde{\mathbf{v}}^{(n+1)}-\nabla \psi^{(n+1)}
$$

onde, por imposição da conservação de massa para $\mathbf{v}^{(n+1)}$, a função $\psi^{(n+1)}$ deve satisfazer a equação de Poisson (3.8). Esse campo de velocidades intermediário é calculado em todas as células $\mathbf{F}$ e $\mathbf{S}$ do domínio. A obtenção de $\widetilde{\mathbf{v}}$ por meio da equação (3.6) envolve a solução de dois sistemas lineares que são resolvidos pelo método dos gradientes conjugados. 
P3. Calcula-se a equação de Poisson para determinar o potencial $\psi$;

$$
\nabla^{2} \psi^{(n+1)}=\nabla \cdot \widetilde{\mathbf{v}}^{(n+1)}
$$

As condições de contorno utilizadas para resolver essa equação em contornos rígidos (B) e entradas de fluidos (I) são as condições homogêneas de Neumann enquanto as condições homogêneas de Dirichlet são aplicadas em saídas de fluidos $(\mathbf{O})$. Essas equações são resolvidas para todas as células cheias $(\mathbf{F})$ do domínio. Entretanto, para manter a estabilidade do esquema numérico quando utiliza-se escoamentos com superfície livre para baixos valores do número adimensional Reynolds, Oishi e colaboradores [13] propuseram uma formulação implícita, utilizando a condição de pressão em superfícies livres. Introduzindo as equações (3.7) e (3.13) em (3.3), obtém-se

$$
\begin{aligned}
p^{(n)}+\frac{\psi^{(n+1)}}{\delta t}-\frac{1}{R e} & \nabla^{2} \psi^{(n+1)}=\frac{2}{R e}\left\{\frac{\partial}{\partial x}\left(\widetilde{u}^{(n+1)}-\frac{\partial \psi^{n+1}}{\partial x}\right) n_{x}^{2}+\right. \\
& {\left[\frac{\partial}{\partial y}\left(\widetilde{u}^{(n+1)}-\frac{\partial \psi^{n+1}}{\partial x}\right)+\frac{\partial}{\partial x}\left(\widetilde{v}^{(n+1)}-\frac{\partial \psi^{n+1}}{\partial y}\right)\right] n_{x} n_{y}+} \\
& \left.\frac{\partial}{\partial y}\left(\widetilde{v}^{(n+1)}-\frac{\partial \psi^{n+1}}{\partial y}\right) n_{y}^{2}\right\}+\Phi^{x x} n_{x}^{2}+2 \Phi^{x y} n_{x} n_{y}+\Phi^{y y} n_{y}^{2}
\end{aligned}
$$

Essa equação é aplicada em todas as células $(\mathbf{S})$ de acordo com a orientação local da superfície livre, que pode ser obtida analisando-se as células vazias (E) vizinhas. Duas aproximações são consideradas:

i) Superfície Planar: Se uma célula $\mathbf{S}$ tem somente uma face em contato com uma célula vazia $\mathbf{E}$ então assume-se que a superfície livre é aproximada por uma reta vertical ou horizontal e neste caso, o vetor normal $\mathbf{n}$ é definido como $(0, \pm 1)^{T}$ ou $( \pm 1,0)^{T}$. Por exemplo, se uma célula $\mathbf{S}$ tem somente a face $\left(i+\frac{1}{2}\right)$ em contato com uma face de uma célula $\mathbf{E}$ (ver Fig. $3.3-(\mathrm{a})$ ), então toma-se $\mathbf{n}=(1,0)^{T}$ e a equação (3.9) se reduz à

$$
\frac{\psi^{(n+1)}}{\delta t}-\frac{1}{R e} \nabla^{2} \psi^{(n+1)}=\frac{2}{R e} \frac{\partial \widetilde{u}^{(n+1)}}{\partial x}-\frac{2}{R e} \frac{\partial^{2} \psi^{n+1}}{\partial x^{2}}+\Phi^{x x}-p^{(n)} .
$$


ii) Superfície inclinada em $45^{\circ}$ : Se uma célula $\mathrm{S}$ tem somente duas faces adjacentes em contato com células vazias $(\mathbf{E})$ então a superfície livre é aproximada por uma reta com inclinação de $45^{\circ}$. Neste caso, o vetor normal é descrito por $\mathbf{n}=\left( \pm \frac{\sqrt{2}}{2}, \pm \frac{\sqrt{2}}{2}\right)$. Por exemplo, considerando a célula $\mathbf{S}$ conforme mostrado na Fig. 3.3 -(b), adota-se $\mathbf{n}=\left(\frac{\sqrt{2}}{2}, \frac{\sqrt{2}}{2}\right)$ e utilizando a equação de conservação de massa, a equação (3.9) se reduz à

$$
\begin{aligned}
\frac{\psi^{(n+1)}}{\delta t} & -\frac{1}{R e} \nabla^{2} \psi^{(n+1)}=\frac{1}{R e}\left\{\frac{\partial \widetilde{u}^{(n+1)}}{\partial y}+\frac{\partial \widetilde{v}^{(n+1)}}{\partial x}+\frac{\partial^{2} \psi^{n+1}}{\partial x^{2}}\right. \\
& \left.+\frac{\partial^{2} \psi^{n+1}}{\partial y^{2}}+2 \frac{\partial^{2} \psi^{n+1}}{\partial x \partial y}\right\}+\frac{1}{2}\left[\Phi^{x x}+2 \Phi^{x y}+\Phi^{y y}\right]-p^{(n)} .
\end{aligned}
$$

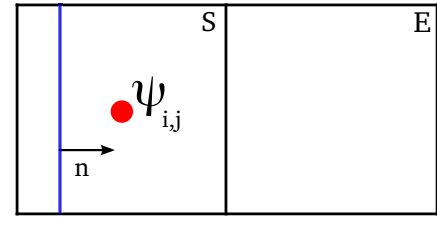

(a) Superfície Planar.

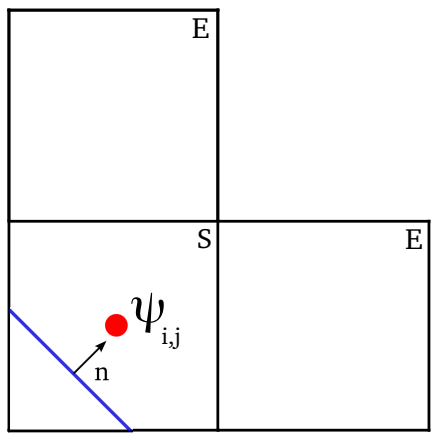

(b) Superfície inclinada de $45^{\circ}$.

Figura 3.3: Aproximações para a superfície livre.

Se uma célula $\mathbf{S}$ apresentar duas faces opostas em contato com células vazias (E) então uma aproximação apropriada para o vetor normal não pode ser atribuída. Para essas células, a função potencial $\psi^{(n+1)}$ é definida como nula. A combinação das equações (3.8) em células cheias $(\mathbf{F})$ e (3.9) em células de superfície livre (S) resulta em um sistema linear não simétrico que é resolvido pelo método dos gradientes bi-conjugados (ver [13]). As condições impostas pela equação (3.4) são as mesmas condições impostas quando utiliza-se, por exemplo, o modelo PTT (Phan-Thien-Tanner - ver [9] ). A aproximação para estas equações pode ser encontrada em detalhes em Paulo e colaboradores [9] e, por este motivo, não está descrita aqui. 
P4. Calcula-se o campo de velocidades final;

Tendo obtido $\psi\left(\mathbf{x}, t_{n+1}\right)$, as velocidades $u\left(\mathbf{x}, t_{n+1}\right)$ e $v\left(\mathbf{x}, t_{n+1}\right)$ em células cheias $(\mathbf{F})$ e em células de superfície livre (S) são calculadas por

$$
\mathbf{v}^{(n+1)}=\widetilde{\mathbf{v}}^{(n+1)}-\nabla \psi^{(n+1)}
$$

P5. Calcula-se o campo de pressão final (ver [13]) substituindo a equação (3.7) em (3.6) e comparando com a discretização da equação de quantidade de movimento por Euler Implícito (3.5), que resulta em

$$
p^{(n+1)}=p^{(n)}+\frac{\psi^{(n+1)}}{\delta t}-\frac{1}{R e} \nabla^{2} \psi^{(n+1)}
$$

Os passos P1.-P5. são resolvidos por aproximações de diferenças finitas de segunda ordem. As discretizações em diferenças finitas para estas equações estão descritas em detalhes em Oishi e colaboradores $[12,13]$ e, por esta razão, não serão apresentadas nesse trabalho.

\subsection{Cálculo do tensor de tensões extra $\boldsymbol{\tau}\left(\mathrm{x}, t_{n+1}\right)$}

Para calcular o tensor de tensões extra $\boldsymbol{\tau}\left(\mathbf{x}, t_{n+1}\right)$ segue-se a metodologia apresentada por Tomé e colaboradores [30]. Nesta metodologia, discretizam-se $(N+1)$ pontos $t_{j}^{\prime}, j=0,1, \cdots, N$, no intervalo $\left[0, t_{n+1}\right]$ e resolve-se a equação constitutiva (2.4) em 3 passos, P6, P7 e P8, como segue:

\section{P6. Discretização do intervalo de tempo $\left[0, t_{n+1}\right]$}

Um das questões mais complexas ao se utilizar o método dos campos de deformação é a distribuição dos nós de integração $0 \leq t_{0}^{\prime}<t_{1}^{\prime}<\cdots<t_{N}^{\prime}=t_{n+1}$ no intervalo [0, $t_{n+1}$ ], uma vez que esta distribuição afeta a precisão dos resultados, principalmente quando se resolvem escoamentos mais complexos.

Hulsen e colaboradores $[44,45]$ usando o conceito de tempo passado $\mathcal{S}=t-t^{\prime}$, definem um ponto de corte nesse passado, $\mathcal{S}_{c}$, e discretizam o intervalo $\left[\mathcal{S}_{c}, t_{n+1}\right]$ para calcular $\boldsymbol{\tau}\left(\mathbf{x}, t_{n+1}\right)$. Esse ponto $\mathcal{S}_{c}$ é definido para que os nós de integração fiquem acumulados perto do tempo atual de simulação; uma vez que a função a ser integrada contém uma função exponencial. Fo- 
ram apresentados resultados de simulações em estado estacionário de um escoamento confinado usando os modelos UCM e KBKZ-PSM. Entretanto, quando se consideram escoamentos em regime transiente com superfícies livres, a aplicação dessa metodologia torna-se mais complicada.

No trabalho de Tomé e colaboradores [30], os nós no intervalo $\left[0, t_{n+1}\right]$ são discretizados usando-se a imagem inversa da função memória em $N$ subintervalos $\left[t_{j-1}^{\prime}, t_{j}^{\prime}\right], j=1,2, \cdots, N$, $\operatorname{com} t_{0}^{\prime}=0, t_{N}^{\prime}=t_{n+1}$ e $t_{j}^{\prime}$ dados por

$$
t_{j}^{\prime}=\frac{\sum_{k=1}^{m_{1}} t_{j_{k}}^{\prime}}{m_{1}}, j=1,2, \cdots, N-1
$$

onde $t_{j_{k}}^{\prime}$ são calculados como

$$
t_{j_{k}}^{\prime}\left(t_{n+1}\right)=\lambda_{k} W i \ln \left(\frac{\lambda_{k} W i}{a_{k}} M_{j_{k}}\left(t_{n+1}\right)\right)+t_{n+1}, \quad k=1,2, \cdots, m_{1}
$$

onde $M_{j_{k}}\left(t_{n+1}\right)=M_{k}\left(t_{n+1}\right)+j \cdot d M_{k}, j=1,2, \cdots, N-1$ e $d M_{k}=\frac{M_{k}(0)-M_{k}\left(t_{n+1}\right)}{N}$ para todo $k$.

Essa metodologia discretiza os nós bem próximos ao tempo presente $t_{n+1}$, e se relaciona com o tempo de resposta do fluido. Essa discretização mostrou-se muito boa para escoamentos confinados de fluidos UCM e KBKZ-PSM, principalmente em escoamentos cisalhantes. Porém, em problemas elongacionais, a imagem inversa da função memória não apresentou bons resultados.

Neste trabalho, propõe-se uma nova metodologia para a discretização dos nós de integração $t_{j}^{\prime}, j=0, \ldots, N$. Essa metodologia usa uma progressão geométrica para distribuir os nós de integração no intervalo $\left[0, t_{n+1}\right]$ para escoamentos dependentes do tempo, da seguinte maneira:

1. Define-se $t_{0}^{\prime}=0$ e $t_{N}^{\prime}=t_{n+1}$;

2. Calcula-se $t_{N-j}^{\prime}=t_{N}^{\prime}-d t q^{j}, j=1,2, \cdots, N-1$, onde $q=\left(\frac{t_{n+1}}{d t}\right)^{1 / N}$.

O passo de tempo dt é o mesmo utilizado para calcular os campos de velocidade e pressão.

Para ilustrar a aplicação desse novo esquema proposto, a Fig. 3.4 apresenta uma distribuição de nós $t_{j}^{\prime}\left(t_{n+1}\right)$ utilizando a progressão geométrica no intervalo $[0,20]$ tomando $N=50,100$ e faz-se a comparação com a imagem inversa da função memória, assumindo-se apenas um modulo de relaxamento, com $M\left(t_{n+1}-t^{\prime}\left(t_{n+1}\right)\right)=100 e^{-\left(t_{n+1}-t^{\prime}\left(t_{n+1}\right)\right)}$. Pode-se verificar que nesse intervalo de tempo $[0,20]$, os pontos $t_{j}^{\prime}\left(t_{n+1}\right)$ discretizados pela imagem inversa da função memória estão restritos ao intervalo [15, 20], mesmo quando se dobram a quantidade de pontos 
na discretização. A progressão geométrica também acumula pontos próximo ao tempo atual de simulação $\left(t_{n+1}=20 \mathrm{~s}\right)$ porém a distribuição é mais suave. Os efeitos desta discretização e sua influência nos resultados serão apresentados na seção de verificação dos resultados.

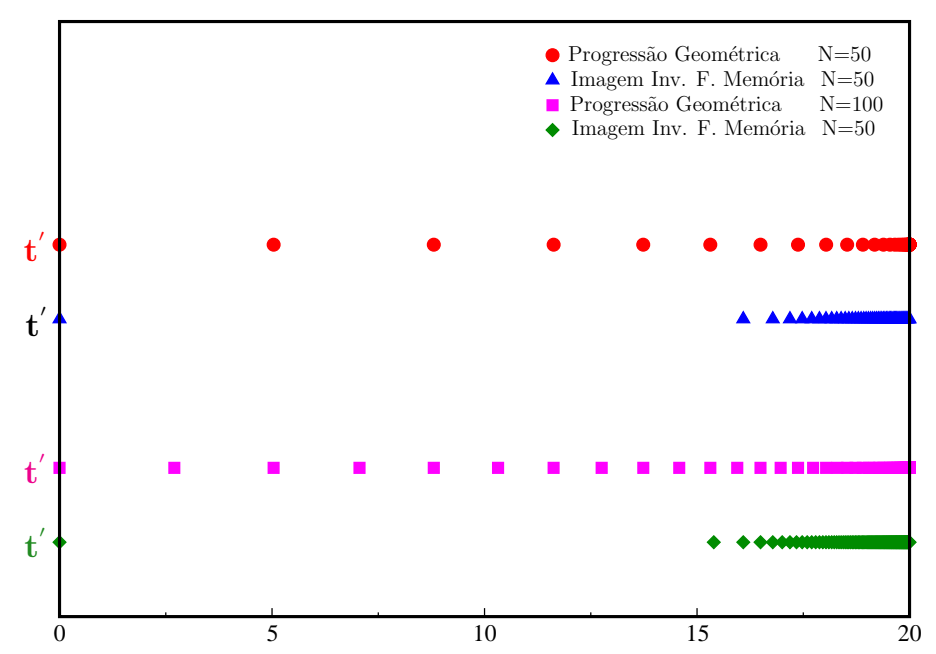

Figura 3.4: Discretização do intervalo [0, 20] em 50 e 100 pontos usando a progressão geométrica e a imagem inversa da função memória.

\section{P7. Cálculo do tensor de Finger $\mathbf{B}_{t_{j}^{\prime}\left(t_{n+1}\right)}\left(\mathbf{x}, t_{n+1}\right)$}

Uma das dificuldades nas simulações numéricas de escoamentos viscoelásticos utilizando equações constitutivas integrais é como calcular o histórico das deformações de maneira precisa. Em elementos finitos, isto pode ser feito utilizando uma formulação baseada no movimento Lagrangiano da partícula (ver [26]). Porém, nesse trabalho, optou-se por uma abordagem diferente, na qual segue-se as ideias do método dos campos de deformação (ver [44,45]) em que o tensor de Finger $\mathbf{B}_{t_{j}^{\prime}\left(t_{n}\right)}\left(\mathbf{x}, t_{n+1}\right)$ é calculado através da seguinte equação de convecção:

$$
\begin{aligned}
\frac{\partial}{\partial t} \mathbf{B}_{t_{j}^{\prime}\left(t_{n}\right)}\left(\mathbf{x}, t_{n+1}\right)+\left(\mathbf{v}\left(\mathbf{x}, t_{n+1}\right) \cdot \nabla\right) \mathbf{B}_{t_{j}^{\prime}\left(t_{n}\right)}\left(\mathbf{x}, t_{n}\right)= & {\left[\nabla \mathbf{v}\left(\mathbf{x}, t_{n+1}\right)\right]^{T} \cdot \mathbf{B}_{t_{j}^{\prime}\left(t_{n}\right)}\left(\mathbf{x}, t_{n}\right) } \\
& +\mathbf{B}_{t_{j}^{\prime}\left(t_{n}\right)}\left(\mathbf{x}, t_{n}\right) \cdot \nabla \mathbf{v}\left(\mathbf{x}, t_{n+1}\right)
\end{aligned}
$$

com a condição $\mathbf{B}_{t_{t_{n+1}}^{\prime}\left(t_{n}\right)}\left(\mathbf{x}, t_{n+1}\right)=\mathbf{I}$.

Para resolver (3.16), escreve-se

$$
\frac{\partial}{\partial t} \mathbf{B}_{t_{j}^{\prime}\left(t_{n}\right)}\left(\mathbf{x}, t_{n+1}\right)=\mathcal{G}[\mathbf{v}, \mathbf{B}], \quad \text { onde }
$$




$$
\begin{aligned}
\mathcal{G}[\mathbf{v}, \mathbf{B}]= & -\left(\mathbf{v}\left(\mathbf{x}, t_{n+1}\right) \cdot \nabla\right) \mathbf{B}_{t_{j}^{\prime}\left(t_{n}\right)}\left(\mathbf{x}, t_{n}\right)+\left[\left(\nabla \mathbf{v}\left(\mathbf{x}, t_{n+1}\right)\right]\right)^{T} \cdot \mathbf{B}_{t_{j}^{\prime}\left(t_{n}\right)}\left(\mathbf{x}, t_{n}\right) \\
& \left.+\mathbf{B}_{t_{j}^{\prime}\left(t_{n}\right)}\left(\mathbf{x}, t_{n}\right) \cdot \nabla \mathbf{v}\left(\mathbf{x}, t_{n+1}\right)\right] .
\end{aligned}
$$

O tensor de Finger $\mathbf{B}_{t_{j}^{\prime}\left(t_{n}\right)}\left(\mathbf{x}, t_{n+1}\right)$ é calculado pelo método de Euler Modificado

$$
\begin{aligned}
\mathbf{B}_{t_{j}^{\prime}\left(t_{n}\right)}\left(\mathbf{x}, t_{n+1}\right)= & \mathbf{B}_{t_{j}^{\prime}\left(t_{n}\right)}\left(\mathbf{x}, t_{n}\right) \\
& +\frac{\delta t}{2}\left\{\mathcal{G}\left[\mathbf{v}^{(n+1)}, \mathbf{B}_{t_{j}^{\prime}\left(t_{n}\right)}\left(\mathbf{x}, t_{n}\right)\right]+\mathcal{G}\left[\mathbf{v}^{(n+1)}, \overline{\mathbf{B}}_{t_{j}^{\prime}\left(t_{n}\right)}\left(\mathbf{x}, t_{n+1}\right)\right]\right\}
\end{aligned}
$$

onde $\overline{\mathbf{B}}_{t_{j}^{\prime}\left(t_{n}\right)}\left(\mathbf{x}, t_{n+1}\right)=\mathbf{B}_{t_{j}^{\prime}\left(t_{n}\right)}\left(\mathbf{x}, t_{n}\right)+\delta t \mathcal{G}\left[\mathbf{v}^{(n+1)}, \mathbf{B}_{t_{j}^{\prime}\left(t_{n}\right)}\left(\mathbf{x}, t_{n}\right)\right]$. O tensor de Finger é calculado para toda célula de superfície livre $(\mathbf{S})$ e célula cheia $(\mathbf{F})$, neste segundo caso, o esquema de alta ordem CUBISTA [51] é aplicado para aproximar os termos convectivos. A aproximação em diferenças finitas para o tensor de Finger e as respectivas condições de contorno estão apresentadas em detalhes em Tomé e colaboradores [30] e não será repetido aqui. Ressalta-se aqui que o tensor de Finger $\mathbf{B}_{t_{j}^{\prime}\left(t_{n}\right)}\left(\mathbf{x}, t_{n+1}\right)$ é convectado no tempo pela equação (3.16) e fornece os valores em $t_{n}$ para cada ponto $t_{j}^{\prime}\left(t_{n}\right)$. Para calcular o valor do tensor de Finger nos novos $t_{j}^{\prime}\left(t_{n+1}\right)$, ou seja, encontrar $\mathbf{B}_{t_{j}^{\prime}\left(t_{n+1}\right)}\left(\mathbf{x}, t_{n+1}\right)$ aplica-se uma interpolação de segunda ordem. A Fig. 3.5 ilustra a ideia aplicada para interpolar o tensor de Finger.

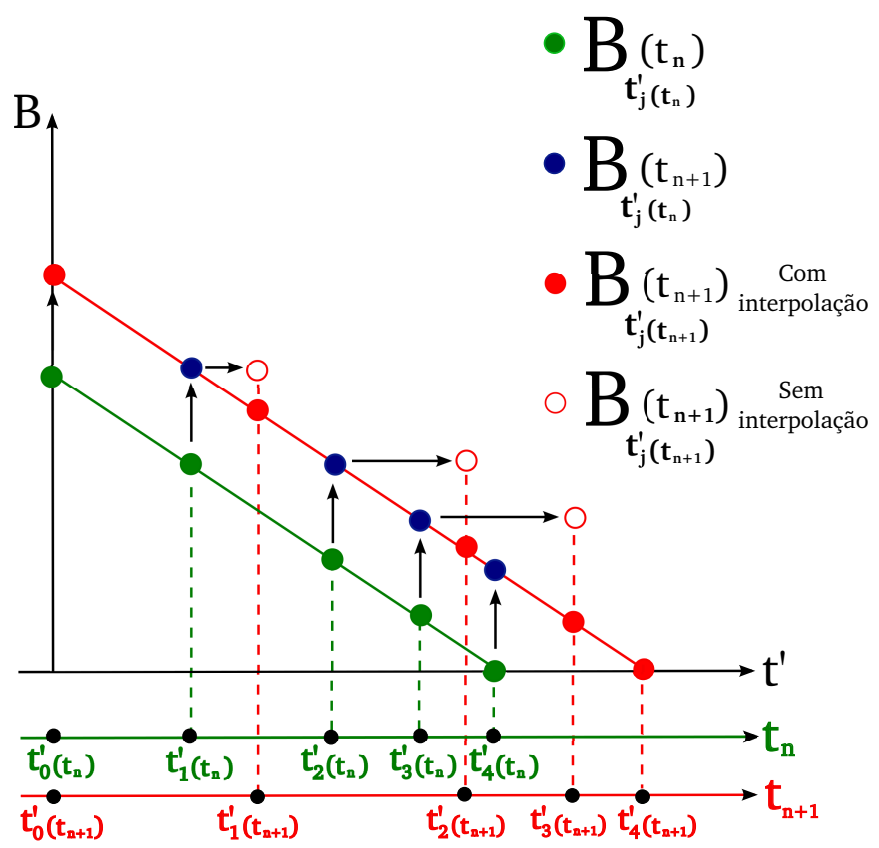

Figura 3.5: Interpolação do tensor de Finger $\mathbf{B}_{t^{\prime}\left(t_{n}\right)}\left(\mathbf{x}, t_{n+1}\right)$ em $t^{\prime}\left(t_{n+1}\right)$. 


\section{P8. Cálculo do tensor $\boldsymbol{\tau}\left(\mathrm{x}, t_{n+1}\right)$}

O intervalo de integração $(-\infty, t]$ é dividido em 2 subintervalos $(-\infty, 0)$ e $[0, t]$. Assim, a equação constitutiva (2.4) é reescrita na forma

$$
\begin{aligned}
\boldsymbol{\tau}\left(\mathbf{x}, \mathbf{t}_{\mathbf{n}+\mathbf{1}}\right)= & \int_{-\infty}^{0} M\left(t_{n+1}-t^{\prime}\right) H\left(I_{1}, I_{2}\right) \mathbf{B}_{t^{\prime}}\left(t_{n+1}\right) d t^{\prime} \\
& +\sum_{j=0}^{\frac{N-2}{2}} \int_{t_{2 j}^{\prime}}^{t_{2 j+2}^{\prime}} M\left(t_{n+1}-t^{\prime}\right) H\left(I_{1}, I_{2}\right) \mathbf{B}_{t^{\prime}}\left(t_{n+1}\right) d t^{\prime}
\end{aligned}
$$

Para $t^{\prime}<0$ assume-se que $\mathbf{B}_{t^{\prime}}\left(t_{n+1}\right)=\mathbf{B}_{0}\left(t_{n+1}\right)$ e a integral simplifica-se como

$$
\int_{-\infty}^{0} M\left(t_{n+1}\right) H\left(I_{1}\left(\mathbf{B}_{0}\left(t_{n+1}\right)\right), I_{2}\left(\mathbf{B}_{0}\left(t_{n+1}\right)\right)\right) \mathbf{B}_{0}\left(t_{n+1}\right) d t^{\prime}
$$

a qual pode ser resolvida exatamente.

Para resolver a soma de integrais na segunda parte da equação (3.20) utiliza-se o método dos coeficientes indeterminados. Assim cada parcela do somatório em (3.20) é aproximada por uma fórmula de quadratura de 3 pontos, como segue:

$$
\begin{aligned}
I_{3}= & A_{0} * H\left(I_{1}\left(\mathbf{B}_{t_{2 j}^{\prime}}\left(t_{n+1}\right)\right), I_{2}\left(\mathbf{B}_{t_{2 j}^{\prime}}\left(t_{n+1}\right)\right)\right) * \mathbf{B}_{t_{2 j}^{\prime}}\left(t_{n+1}\right) * M\left(t_{n+1}-t_{2 j}^{\prime}\right) \\
& +A_{1} * H\left(I_{1}\left(\mathbf{B}_{t_{2 j+1}^{\prime}}\left(t_{n+1}\right)\right), I_{2}\left(\mathbf{B}_{t_{2 j+1}^{\prime}}\left(t_{n+1}\right)\right)\right) * \mathbf{B}_{t_{2 j+1}^{\prime}}\left(t_{n+1}\right) * M\left(t_{n+1}-t_{2 j+1}^{\prime}\right) \\
& +A_{2} * H\left(I_{1}\left(\mathbf{B}_{t_{2 j+2}^{\prime}}\left(t_{n+1}\right)\right), I_{2}\left(\mathbf{B}_{t_{2 j+2}^{\prime}}\left(t_{n+1}\right)\right)\right) * \mathbf{B}_{t_{2 j+2}^{\prime}}\left(t_{n+1}\right) * M\left(t_{n+1}-t_{2 j+2}^{\prime}\right) .
\end{aligned}
$$

Os coeficientes $A_{0}, A_{1}, A_{2}$ são obtidos resolvendo-se o sistema linear $(3 \times 3)$

$$
\begin{aligned}
A_{0}+A_{1}+A_{2} & =b_{0}=\int_{t_{2 j}^{\prime}}^{t_{2 j+2}^{\prime}} d t^{\prime}, \\
A_{0} * t_{2 j}^{\prime}+A_{1} * t_{2 j+1}^{\prime}+A_{2} * t_{2 j+2}^{\prime} & =b_{1}=\int_{t_{2 j}^{\prime}}^{t_{2 j+2}^{\prime}} t^{\prime} d t^{\prime}, \\
A_{0} *\left(t_{2 j}^{\prime}\right)^{2}+A_{1} *\left(t_{2 j+1}^{\prime}\right)^{2}+A_{2} *\left(t_{2 j+2}^{\prime}\right)^{2} & =b_{2}=\int_{t_{2 j}^{\prime}}^{t_{2 j+2}^{\prime}} t^{\prime 2} d t^{\prime},
\end{aligned}
$$


e assim,

$$
\begin{aligned}
& A_{2}=\frac{t_{2 j+1}^{\prime} t_{2 j}^{\prime} b_{0}+b_{2}-t_{2 j}^{\prime} b_{1}-t_{2 j+1}^{\prime} b_{1}}{\left(t_{2 j+2}^{\prime}\right)^{2}-t_{2 j+1}^{\prime} t_{2 j+2}^{\prime}+t_{2 j+1}^{\prime} t_{2 j}^{\prime}-t_{2 j}^{\prime} t_{2 j+2}^{\prime}}, \\
& A_{1}=\frac{-t_{2 j}^{\prime} b_{1}+t_{2 j}^{\prime} b_{0} t_{2 j+2}^{\prime}+b_{2}-t_{2 j+2}^{\prime} b_{1}}{\left(t_{2 j+1}^{\prime}-t_{2 j}^{\prime}\right)\left(t_{2 j+1}^{\prime}-t_{2 j+2}^{\prime}\right)}, \\
& A_{0}=-\frac{-t_{2 j+1}^{\prime} b_{1}+t_{2 j+1}^{\prime} b_{0} t_{2 j+2}^{\prime}+b_{2}-t_{2 j+2}^{\prime} b_{1}}{\left(-t_{2 j+2}^{\prime}+t_{2 j}^{\prime}\right)\left(t_{2 j+1}^{\prime}-t_{2 j}^{\prime}\right)} .
\end{aligned}
$$

\section{P9. Movimentação das partículas marcadoras}

O ultimo passo do algoritmo é a atualização da posição da superfície livre. A superfície livre é descrita por um conjunto de partículas marcadoras e visualizada pela união destes pontos (ver Fig. 3.2). Essas partículas marcadoras se movem no tempo resolvendo-se a equação

$$
\frac{d \mathbf{x}_{P}}{d t}=\mathbf{v}^{(n+1)}
$$

para toda partícula $\mathbf{x}_{P}$ usando o método de Euler Modificado,

$$
\mathbf{x}_{P}\left(t_{n+1}\right)=\mathbf{x}_{P}\left(t_{n}\right)+\frac{\delta t}{2}\left[\mathbf{v}\left(\mathbf{x}_{P}\left(t_{n}\right), t_{n+1}\right)+\overline{\mathbf{v}}\left(\overline{\mathbf{x}}_{P}\left(t_{n+1}\right), t_{n+1}\right)\right]
$$

onde $\overline{\mathbf{x}}_{P}\left(t_{n+1}\right)=\mathbf{x}_{P}\left(t_{n}\right)+\delta t \mathbf{v}\left(\mathbf{x}_{P}\left(t_{n}\right), t_{n+1}\right)$.

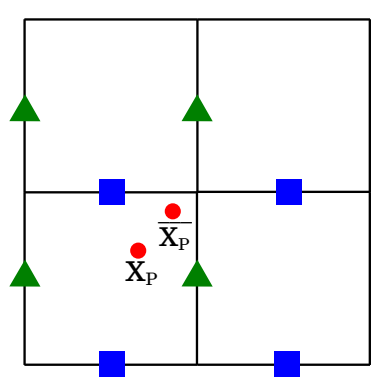

Figura 3.6: Ilustração das velocidades envolvidas no cálculo da velocidade da partícula $\mathbf{v}\left(\mathbf{x}_{P}\left(t_{n}\right), t_{n+1}\right)=\left(u\left(\mathbf{x}_{P}\left(t_{n}\right), t_{n+1}\right), v\left(\mathbf{x}_{P}\left(t_{n}\right), t_{n+1}\right)\right)$ usando interpolação bilinear. A velocidade $u\left(\mathbf{x}_{P}\left(t_{n}\right), t_{n+1}\right)$ é obtida usando as velocidades representadas pelos símbolos $\boldsymbol{\Delta}$ enquanto $v\left(\mathbf{x}_{P}\left(t_{n}\right), t_{n+1}\right)$ envolve as velocidades exemplificadas em 
As velocidades $\mathbf{v}\left(\mathbf{x}_{P}\left(t_{n}\right), t_{n+1}\right)$ e $\overline{\mathbf{v}}\left(\overline{\mathbf{x}}_{P}\left(t_{n+1}\right), t_{n+1}\right)$ são calculadas usando o campo de velocidades atualizado, $\mathbf{v}^{(n+1)}$, e são obtidas por uma interpolação bilinear envolvendo as quatro células vizinhas mais próximas e suas correspondentes distâncias. Podem acontecer situações em que duas partículas contíguas fiquem muito distantes ou excessivamente juntas. Nestes casos, um algoritmo para inserir ou deletar partículas é aplicado ( para detalhes, ver [48]). A Fig. 3.6 ilustra as velocidades envolvidas no cálculo da velocidade da partícula $\mathbf{v}\left(\mathbf{x}_{P}\left(t_{n}\right), t_{n+1}\right)$.

As equações descritas nas seções anteriores foram discretizadas pelo método de diferenças finitas de segunda ordem no espaço e no tempo. A discretização das equações em diferenças finitas é similar às apresentadas em detalhes em $[12,13,30]$ e não serão reapresentadas aqui. 


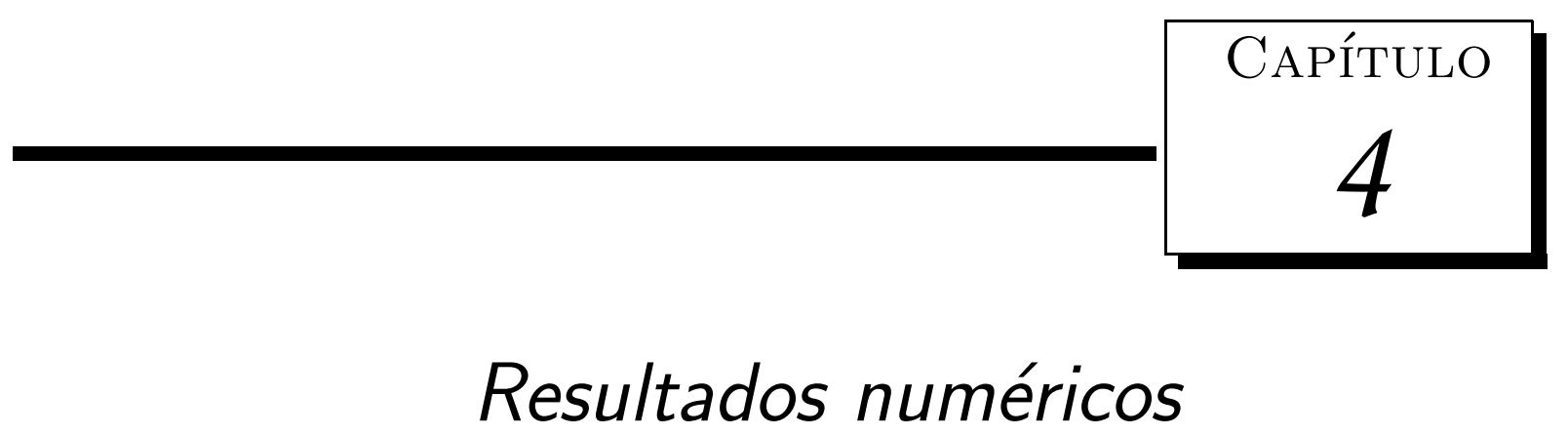

Neste capítulo, apresentam-se resultados numéricos provenientes da aplicação da metodologia descrita nos capítulos anteriores na solução de escoamentos incompressíveis. Inicialmente, considera-se escoamentos de fluidos UCM e apresentam-se resultados de verificação da nova discretização dos pontos $t_{j}^{\prime}$ utilizando a progressão geométrica, e efetua-se uma comparação com as metodologias propostas por Tomé e colaboradores [30] e Hulsen e colaboradores [45]. Em seguida, utilizando a distribuição via progressão geométrica, resultados para validação em escoamentos confinados e com superfícies livres de fluidos KBKZ são apresentados. Finalmente, resultados numéricos para os problemas conhecidos como Jet Buckling e Extrudate-Swell são apresentados.

\subsection{Verificação da nova distribuição dos pontos $t_{j}^{\prime}$}

Para verificar a eficiência da metodologia desenvolvida para a discretização dos nós de integração $t_{j}^{\prime}$ descrita na seção 3.2, utiliza-se a simulação de escoamentos cisalhantes e elongacionais de um fluido UCM. O modelo KBKZ-PSM se reduz ao modelo UCM, tomando $m_{1}=1 \mathrm{e}$ $H\left(I_{1}, I_{2}\right)=1$ na equação (2.4). Neste caso, conhece-se a solução analítica, que é descrita como segue. 
Solução exata para o fluido UCM em escoamento simples de cisalhamento: Neste caso, o campo de velocidades e o tensor de Finger são escritos exatamente como :

$$
\left\{\begin{array}{l}
u=\dot{\gamma} y, \\
v=0,
\end{array} \quad \text { e } \quad \mathbf{B}_{t^{\prime}}(\mathbf{x}, t)=\left(\begin{array}{ccc}
1+\dot{\gamma}^{2}\left(t-t^{\prime}\right)^{2} & \dot{\gamma}\left(t-t^{\prime}\right) & 0 \\
\dot{\gamma}\left(t-t^{\prime}\right) & 1 & 0 \\
0 & 0 & 1
\end{array}\right)\right.
$$

e assim os tensores de tensão extra são dados por

$$
\begin{aligned}
\tau^{x x} & =a e^{\frac{-t}{\lambda}}\left(1+\dot{\gamma}^{2} t^{2}\right)+a\left(1-e^{\frac{-t}{\lambda}}\right)+a \dot{\gamma}^{2}\left[2 \lambda^{2}-e^{\frac{-t}{\lambda}}\left(t^{2}+2 \lambda t+2 \lambda^{2}\right)\right], \\
\tau^{x y} & =a \dot{\gamma} \lambda\left(1-e^{\frac{-t}{\lambda}}\right), \\
\tau^{y y} & =a .
\end{aligned}
$$

Solução exata para o fluido UCM em escoamento extensional uniaxial: Nesses escoamentos, o campo de velocidades e o tensor de Finger são dados como :

$$
\left\{\begin{array}{l}
u=\dot{\varepsilon} x, \\
v=-\frac{1}{2} \dot{\varepsilon} y,
\end{array} \quad \text { e } \quad \mathbf{B}_{t^{\prime}}(\mathbf{x}, t)=\left(\begin{array}{ccc}
e^{2 \dot{\varepsilon}\left(t-t^{\prime}\right)} & 0 & 0 \\
0 & e^{-\dot{\varepsilon}\left(t-t^{\prime}\right)} & 0 \\
0 & 0 & e^{-\dot{\varepsilon}\left(t-t^{\prime}\right)}
\end{array}\right)\right.
$$

Pode-se mostrar que os tensores de tensões extra $\tau^{x x}$ e $\tau^{y y}$ são obtidos pelas equações

$$
\begin{aligned}
\tau^{x x} & =a e^{\left(\frac{-t(1-2 \lambda \dot{\varepsilon})}{\lambda}\right)}\left[1-\frac{1}{1-2 \lambda \dot{\varepsilon}}\right]+\frac{a}{1-2 \lambda \dot{\varepsilon}}, \\
\tau^{y y} & =a e^{\left(-\frac{t(1+\lambda \dot{\varepsilon})}{\lambda}\right)}\left[1-\frac{1}{1+\lambda \dot{\varepsilon}}\right]+\frac{a}{1+\lambda \dot{\varepsilon}} .
\end{aligned}
$$

Nas equações (4.1)-(4.7), $\dot{\gamma}$ e $\dot{\varepsilon}$ denotam a razão de cisalhamento e elongacional enquanto $\lambda$ e a são o tempo de relaxamento do fluido e o modulo de relaxamento, respectivamente. Para valores negativos de $t^{\prime}$ em $(-\infty, 0]$ o tensor de Finger assume o valor do tensor em $t_{0}^{\prime}=0$.

Para comparar a discretização dos $t_{j}^{\prime}$, o tensor de Finger é calculado pelas as equações (2.18)-(2.20), simplificando-as para os casos de escoamentos puramente elongacionais ou cisalhantes. Assim, as equações para calcular as componentes do tensor de Finger são escritas simplificadamente como 
ESCOAMENTOS CISALHANTES

$$
\left\{\begin{array}{l}
\frac{\partial}{\partial t} B_{t^{\prime}}^{y y}=0, \\
\frac{\partial}{\partial t} B_{t^{\prime}}^{x y}=\dot{\gamma} B_{t^{\prime}}^{y y}, \\
\frac{\partial}{\partial t} B_{t^{\prime}}^{x x}=2 \dot{\gamma} B_{t^{\prime}}^{x y} .
\end{array}\right.
$$

ESCOAMENTOS ELONGACIONAIS

$$
\left\{\begin{array}{l}
\frac{\partial}{\partial t} B_{t^{\prime}}^{x x}=2 \dot{\varepsilon} B_{t^{\prime}}^{x x}, \\
\frac{\partial}{\partial t} B_{t^{\prime}}^{y y}=-\dot{\varepsilon} B_{t^{\prime}}^{y y} .
\end{array}\right.
$$

Em escoamentos elongacionais, o tensor $\tau^{x x}$ exibe uma singularidade em $\lambda \dot{\varepsilon}=\frac{1}{2}$, assim, os valores atribuídos para $\lambda \dot{\varepsilon}$ são tais que $\lambda \dot{\varepsilon}<\frac{1}{2}$. Para mostrar o efeito da discretização do intervalo $\left[0, t_{n+1}\right]$ usando a progressão geométrica, resolveu-se numericamente as integrais da equação (2.4) pelo método dos coeficientes indeterminados e calculou-se o tensor de tensões extra, para escoamentos elongacionais e cisalhantes. Os dados utilizados nesta simulação foram $a=100 \mathrm{~Pa}, \lambda=1 \mathrm{~s}, t_{n+1}=10 \lambda, \dot{\gamma}=1 \mathrm{~s}^{-1}, \dot{\varepsilon}=0.3 \mathrm{~s}^{-1}, \delta t=10^{-6} \mathrm{~s}$ (usado para calcular o tensor de Finger pela equação (4.8)). As componentes da tensão foram calculadas numericamente usando a distribuição dos $t_{j}^{\prime}$ obtida pela imagem inversa da função memória e a metodologia que emprega uma progressão geométrica. Os valores analíticos das componentes do tensor foram utilizados para calcular os erros relativos $\left(\left|\tau_{\text {exact }}^{x x}-\tau_{\text {num }}^{x x}\right| /\left|\tau_{\text {exact }}^{x x}\right|\right)$. A Fig. 4.1 exibe os erros relativos para escoamentos cisalhantes e elongacionais para alguns valores de $N$. A solução apresentada por Hulsen e colaboradores [45] para $N=20,40,80$ é também exibida. Para melhor análise dos dados, apresenta-se ainda os resultados desses erros na tabela 4.1.

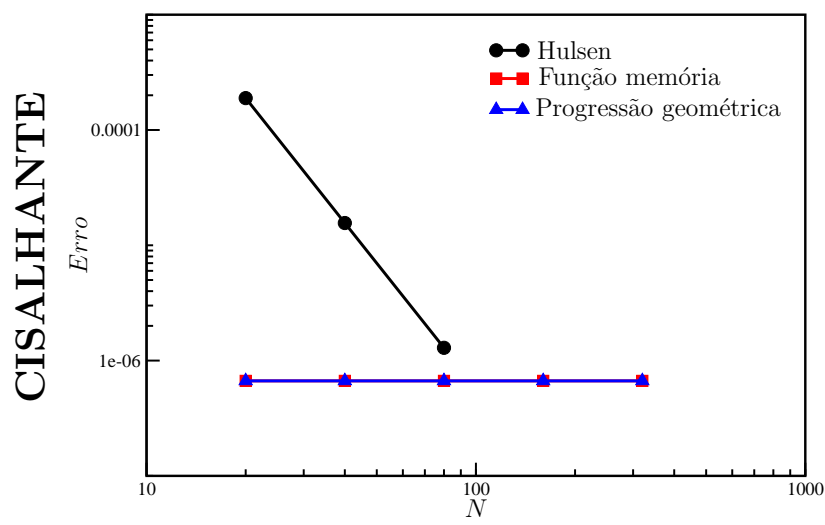

(a)

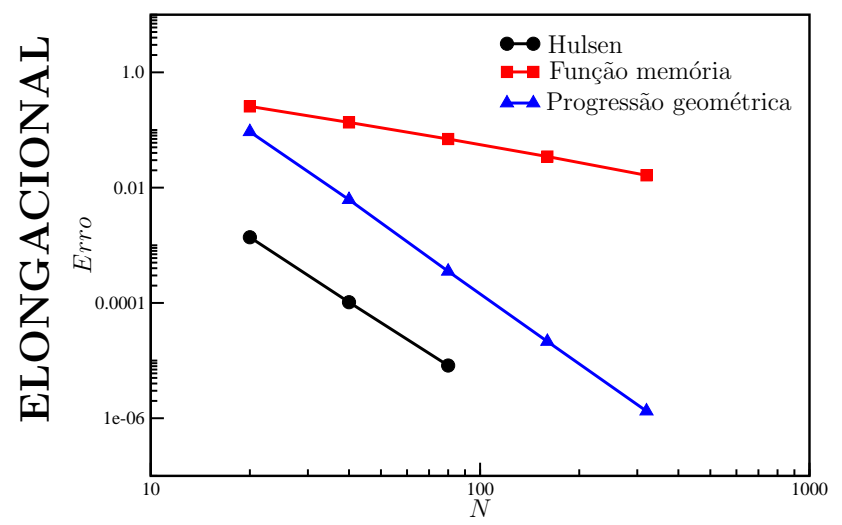

(b)

Figura 4.1: Erros relativos no cálculo de $\tau^{x x}$ em $t=10 \lambda$ como função de $N$ usando $\dot{\gamma}=1 \mathrm{~s}^{-1}$ e $\dot{\varepsilon}=0.3 \mathrm{~s}^{-1}$ para escoamentos cisalhantes (a) e elongacionais (b). 
Tabela 4.1: Erros relativos para o cálculo de $\tau^{x x}$ considerando escoamentos elongacionais e cisalhantes em função de $N$.

\begin{tabular}{|c|c|c|c|c|}
\hline $\mathbf{N}$ & Cisalhante & & Elongacional & \\
\hline \multirow[t]{3}{*}{20} & Hulsen e colaboradores & $1.8900 \times 10^{-4}$ & Hulsen e colaboradores & $1.3800 \times 10^{-4}$ \\
\hline & Função Memória & $6.6685 \times 10^{-7}$ & Função Memória & $2.5664 \times 10^{-1}$ \\
\hline & Progressão geométrica & $6.6685 \times 10^{-7}$ & Progressão geométrica & $9.3170 \times 10^{-2}$ \\
\hline \multirow[t]{3}{*}{40} & Hulsen e colaboradores & $1.5600 \times 10^{-5}$ & Hulsen e colaboradores & $1.0000 \times 10^{-4}$ \\
\hline & Função Memória & $6.6685 \times 10^{-7}$ & Função Memória & $1.3545 \times 10^{-1}$ \\
\hline & Progressão geométrica & $6.6685 \times 10^{-7}$ & Progressão geométrica & $6.1800 \times 10^{-3}$ \\
\hline \multirow[t]{3}{*}{80} & Hulsen e colaboradores & $1.2900 \times 10^{-6}$ & Hulsen e colaboradores & $8.2000 \times 10^{-6}$ \\
\hline & Função Memória & $6.6685 \times 10^{-7}$ & Função Memória & $6.97 \times 10 \times 10^{-2}$ \\
\hline & Progressão geométrica & $6.6685 \times 10^{-7}$ & Progressão geométrica & $3.5000 \times 10^{-4}$ \\
\hline \multirow[t]{3}{*}{160} & Hulsen e colaboradores & ----- & Hulsen e colaboradores & ----- \\
\hline & Função Memória & $6.6685 \times 10^{-7}$ & Função Memória & $3.4620 \times 10^{-2}$ \\
\hline & Progressão geométrica & $6.6685 \times 10^{-7}$ & Progressão geométrica & $2.1438 \times 10^{-5}$ \\
\hline \multirow[t]{3}{*}{320} & Hulsen e colaboradores & ----- & Hulsen e colaboradores & ---- \\
\hline & Função Memória & $6.6685 \times 10^{-7}$ & Função Memória & $1.6340 \times 10^{-2}$ \\
\hline & Progressão geométrica & $6.6685 \times 10^{-7}$ & Progressão geométrica & $1.3290 \times 10^{-6}$ \\
\hline
\end{tabular}

Pode-se ver na Fig. 4.1-(a) que o erro relativo para escoamentos cisalhantes é o mesmo utilizando a imagem inversa da função memória e a progressão geométrica. Isso justifica-se pois a fórmula de quadratura aplicada para resolver a integral tem segunda ordem de precisão e assim, para este tipo de escoamento simples, resulta em um valor exato. O erro obtido de $6.6685 \times 10^{-7}$ (ver tabela 4.1) advém do emprego do método de Euler explícito, que é de primeira ordem, utilizado no cálculo do tensor de Finger. Com relação à escoamentos elongacionais, a 
Fig. 4.1-(b) mostra que os erros obtidos com a progressão geométrica são menores que os obtidos com a imagem inversa da função memória, e apresenta resultados similares aos apresentados por Hulsen e colaboradores [45].

Para melhor ilustrar os efeitos da distribuição dos nós de integração $t_{j}^{\prime}$ no cálculo de tensor de tensões extra, a componente $\tau^{x x}$ foi calculada por ambas as técnicas, para a discretização do intervalo $[0,10]$. A simulação foi realizada até $t_{n+1}=10$ s usando $N=30,50$ para $\dot{\varepsilon}$ no intervalo $[0.1,0.49]$. O erro relativo é plotado na Fig. 4.2 , onde pode-se verificar que para valores pequenos de $\dot{\varepsilon}$ as duas técnicas apresentam erros similares. Entretanto, conforme $\dot{\varepsilon}$ cresce e se aproxima do valor de singularidade $\dot{\varepsilon}=0.5$ os erros cometidos pela progressão geométrica são muito menores que os erros cometidos ao se utilizar a imagem inversa da função memória. Esses resultados sugerem que para problemas mais complexos, a distribuição de pontos utilizando a progressão geométrica fornece melhores resultados que a distribuição de pontos obtida com a imagem inversa da função memória.

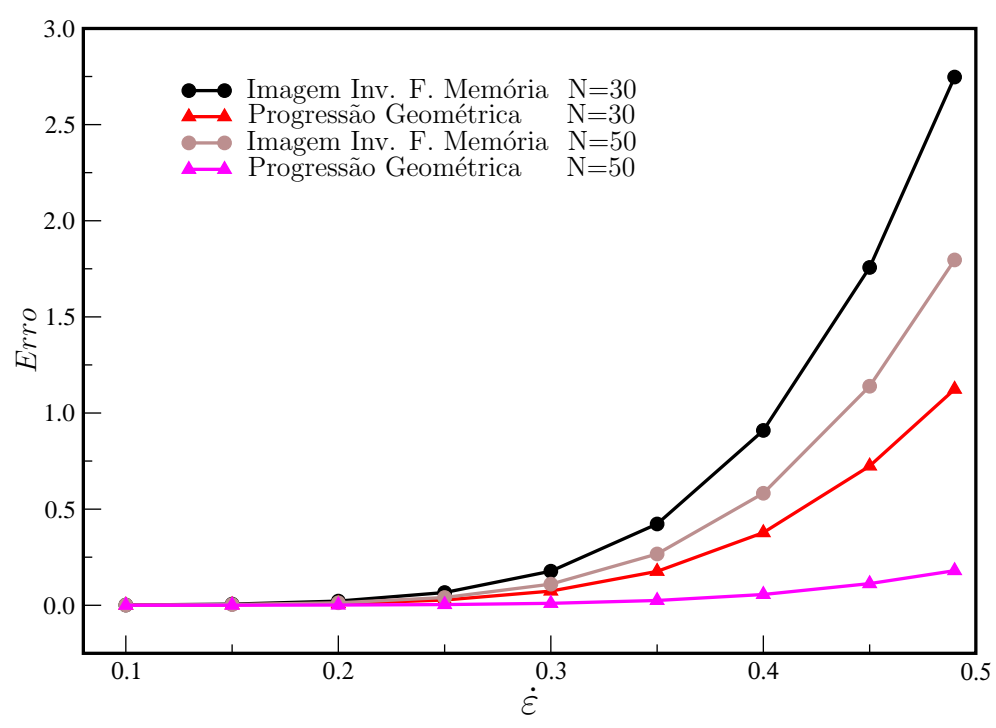

Figura 4.2: Erros no cálculo de $\tau^{x x}$ para $0.1 \leq \dot{\varepsilon} \leq 0.49, \lambda=1 \mathrm{~s}, a=100 \mathrm{~Pa}$ usando a imagem inversa da função memória (círculos) e a progressão geométrica (triângulos) para $N=30,50$.

Nos resultados numéricos apresentados nas próximas seções, a discretização dos pontos $t_{j}^{\prime}$, nos quais armazena-se o histórico de deformação, foi realizada utilizando-se a progressão geométrica, para escoamentos confinados e com superfícies livres em movimento. 


\subsection{Escoamentos confinados}

Nessa seção, a técnica numérica descrita nas seções anteriores é aplicada em dois problemas de escoamentos confinados entre placas paralelas. Um escoamento de fluido KBKZ com um único módulo de relaxamento é simulado e a solução obtida em várias malhas é comparada com a solução analítica que é descrita em detalhes em Tomé e colaboradores (ver apêndice A). Um segundo escoamento entre placas paralelas é simulado, desta vez utilizando-se o fluido multi-módulo denominado Fluido S1 (ver [54]) em várias malhas.

\subsubsection{Verificação em um canal para fluido KBKZ-PSM com 1 módulo de relaxamento}

O escoamento entre placas paralelas foi simulado utilizando um fluido KBKZ com 1 módulo de relaxamento. Para esse fluido, utilizou-se $\lambda_{1}=\lambda_{\text {ref }}=0.1396 \mathrm{~s}, a_{1}=1.6648 \mathrm{~Pa}, \rho_{0}=$ $801.5 \mathrm{~kg} \mathrm{~m}^{-3}$ e $\eta_{0}=\lambda_{1} * a_{1}=0.2324$ Pa.s, $U=0.025 \mathrm{~ms}^{-1}$ e $L=1 \mathrm{~cm}$, o que fornece $R e=$ $\frac{\rho_{0} U L}{\eta_{0}}=0.8621$ e $W i=\lambda_{\text {ref }} \frac{U}{L}=0.3490$. Esse escoamento foi simulado nas malhas $I(100 \times 10)$, $I I(200 \times 20), I I I(300 \times 30)$ e $I V(400 \times 40)$ até o tempo final $t=100$ s. Os resultados obtidos e as respectivas soluções analíticas para $\partial u(y) / \partial y, \tau^{x x}$ e $\tau^{y y}$ são apresentados na Fig. 4.3. Pode-se observar que existe boa concordância entre os resultados numéricos e as soluções analíticas que são apresentadas em detalhes no apêndice A. Observa-se também que a solução numérica converge para a solução analítica quando a malha é refinada. Para quantificar esta convergência, os erros relativos obtidos nas malhas $M_{l}$ (onde $l$ denota $I, I I, I I I$ ou $I V$ ) foram calculados pela fórmula (ver [55])

$$
E\left(M_{l}\right)=\sqrt{H_{l} \sum_{j=1}^{J_{l}}\left[\operatorname{Sol}_{a n}\left(M_{l}\right)_{j}-\operatorname{Sol}_{n u m}\left(M_{l}\right)_{j}\right]^{2}},
$$

onde $J_{l}$ denota o valor $j_{\max }$ na malha $M_{l}$ e $H_{l}$ é o espaçamento de malha $d y$.

Os erros foram calculados usando os valores em uma seção transversal do canal antes da região de saída de fluido. Esses erros são apresentados na tabela 4.2 onde pode-se ver que os erros relativos diminuem quando a malha é refinada, decaindo para menos de $1 \%$ na malha mais fina. Adicionalmente, o gradiente de pressão foi calculado numericamente (ver última coluna da tabela 4.2) e os resultados mostram convergência para o valor analítico. 
A ordem de convergência entre as malhas, foi calulada pela equação (ver [55])

$$
O\left(M_{l}, M_{l+1}\right)=\frac{\log \left(E\left(M_{l}\right) / E\left(M_{l+1}\right)\right)}{\log \left(H_{l} / H_{l+1}\right)}
$$

e está indicada na tabela 4.3, que mostra valores menores que o valor teórico 2, desde que foram utilizadas aproximações de 2a. ordem nas equações de diferenças finitas. Esta diferença pode ser justificada pelo fato de que os cálculos dos erros envolvem pontos próximos ao contorno do domínio, onde emprega-se aproximações de primeira ordem. Ainda, para calcular a solução analítica nos pontos de malha, utilizou-se a fórmula de integração 1/3 de Simpson e interpolação linear. Estas aproximações podem ter afetado a precisão dos resultados numéricos produzindo valor abaixo de 2 .

Tabela 4.2: Erros relativos de $\frac{\partial u(y)}{\partial y}, \tau^{x x}, \tau^{y y}$, obtidos nas malhas $I, I I, I I I, I V$. A última coluna mostra o valor médio para $p_{x} \approx\left(\sum_{j=1}^{J_{l}} \frac{p_{i_{\text {out }}, j}-p_{i_{\text {out }-1, j}}}{\delta x}\right) / J_{l}$, onde $i_{\text {out }}$ denota o índice da célula mais próxima à saída do canal.

\begin{tabular}{ccccc}
\hline \hline Malha $M_{l}$ & $\frac{\partial u(y)}{\partial y}$ & $\tau^{x x}(y)$ & $\tau^{y y}(y)$ & $\begin{array}{c}\text { Valor exato } \\
p_{x}=-6.42226\end{array}$ \\
\hline \hline$I$ & $0.26944 \mathrm{E}-01$ & $0.41842 \mathrm{E}-01$ & $0.48030 \mathrm{E}-02$ & -6.32040 \\
\hline$I I$ & $0.87159 \mathrm{E}-02$ & $0.13173 \mathrm{E}-01$ & $0.16279 \mathrm{E}-02$ & -6.37054 \\
\hline$I I I$ & $0.42141 \mathrm{E}-02$ & $0.61982 \mathrm{E}-02$ & $0.88220 \mathrm{E}-03$ & -6.39534 \\
\hline$I V$ & $0.26414 \mathrm{E}-02$ & $0.37813 \mathrm{E}-02$ & $0.63113 \mathrm{E}-03$ & -6.40965 \\
\hline \hline
\end{tabular}

Tabela 4.3: Ordens de convergência obtidas nas malhas $I, I I, I I I, I V$ usando os dados apresentados na Tabela 4.2

\begin{tabular}{cccc}
\hline \hline Malha & $\frac{\partial u(y)}{\partial y}$ & $\tau^{x x}(y)$ & $\tau^{y y}(y)$ \\
\hline \hline$I-I I$ & 1.628 & 1.667 & 1.561 \\
\hline$I I-I I I$ & 1.792 & 1.859 & 1.511 \\
\hline$I I I-I V$ & 1.624 & 1.718 & 1.164 \\
\hline \hline
\end{tabular}




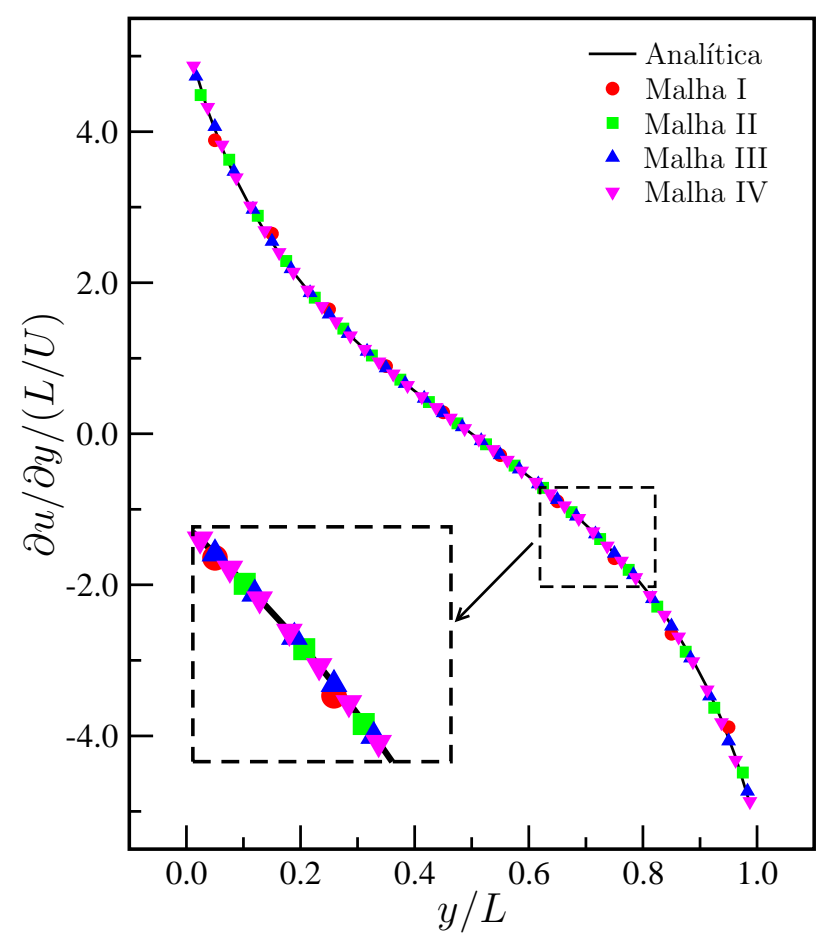

(a)

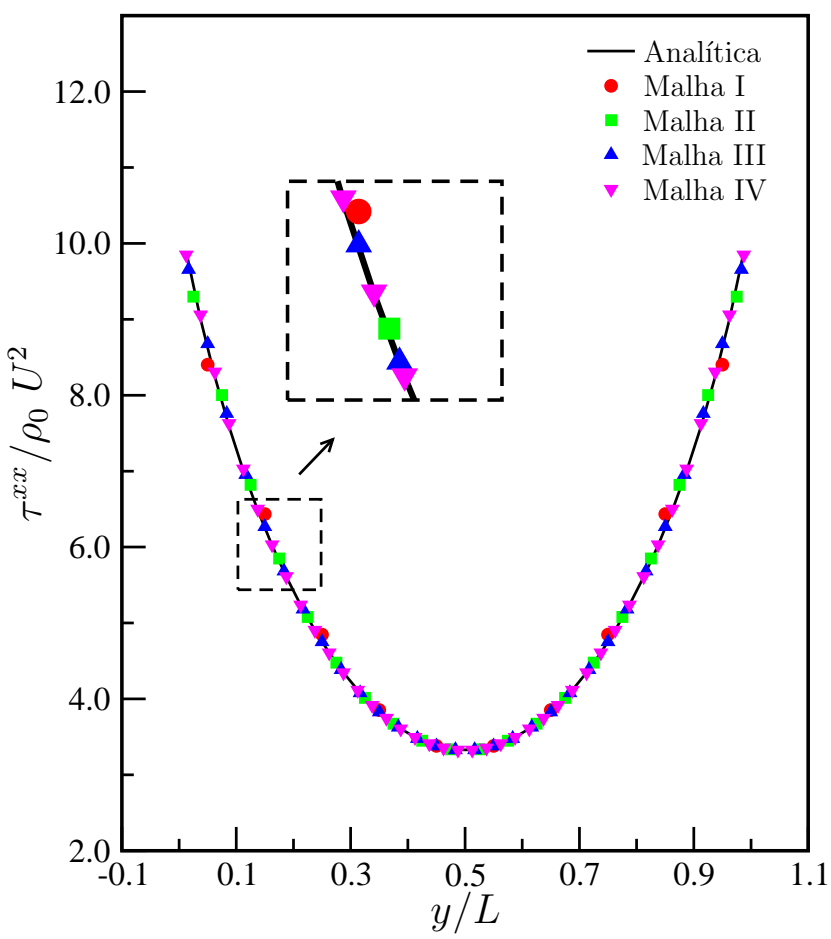

(b)

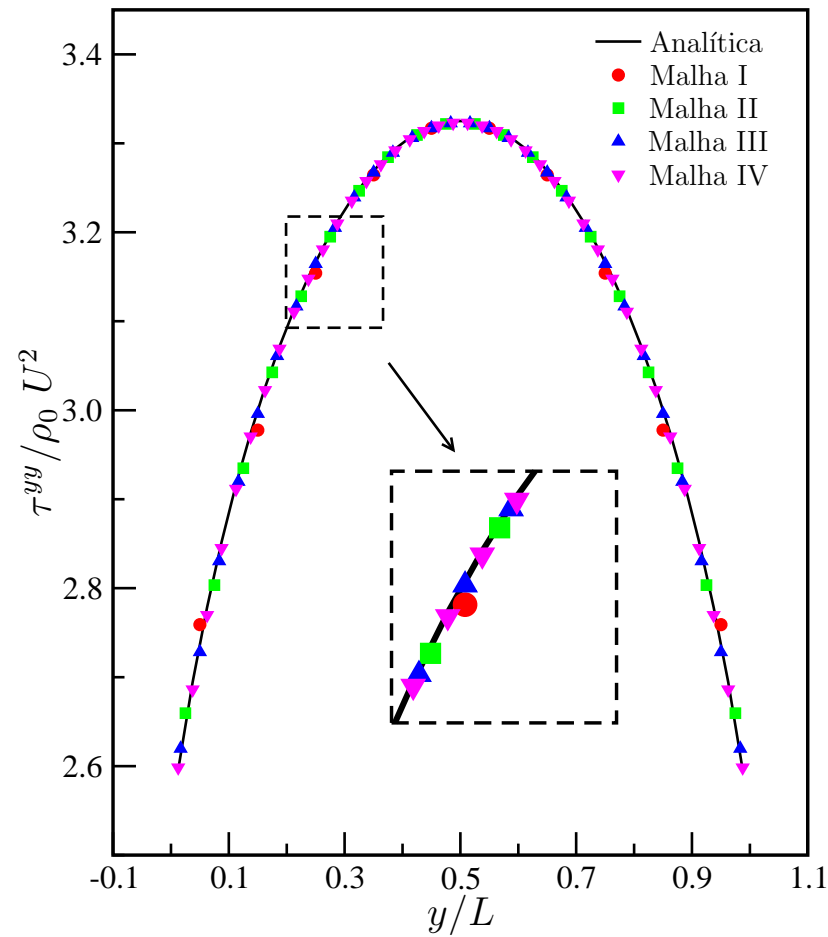

(c)

Figura 4.3: Solução numérica em um canal usando fluido KBKZ com 1 módulo de relaxamento. Comparação de $\partial u / \partial y /(L / U)(\mathrm{a}), \tau^{x x}(y) /\left(\rho_{0} U^{2}\right)(\mathrm{b})$ e $\tau^{y y}(y) /\left(\rho_{0} U^{2}\right)$ (c) obtidas nas malhas $I, I I, I I I, I V$ em comparação com a respectiva solução analítica 


\subsubsection{Verificação em um canal para fluido KBKZ-PSM multi-módulos}

Nesta seção apresenta-se a simulação do escoamento de um fluido KBKZ em um canal com comprimento $10 L$ e largura $L=1 \mathrm{~cm}$. Na entrada do canal, impôs-se um perfil de velocidade Newtoniano dado por

$$
u(y / L)=-4(y / L-0.5)^{2}+1
$$

A tolerância para a solução da equação de Poisson foi $E P S=10^{-10}$, utilizou-se $N=100$ campos de deformação e $U=0.167 \mathrm{~m} / \mathrm{s}$. Os dados reológicos utilizados foram do fluido $S 1$ (ver [54]) que são apresentados na tabela 4.4 .

Tabela 4.4: Definição do fluido empregado na simulação do escoamento em um canal (obtidos de Quinzani [54]).

\begin{tabular}{|c|c|c|c|}
\hline \multicolumn{4}{|c|}{ FLUIDOS1 } \\
\hline \multicolumn{4}{|c|}{$\rho_{0}=801.5 \mathrm{~kg} / \mathrm{m}^{3}, \alpha=10, \beta=0.7, \lambda_{\text {ref }}=0.06 \mathrm{~s}, \eta_{0}=1.424 \mathrm{~Pa} . \mathrm{s}$} \\
\hline & $k \quad \lambda_{k}[\mathrm{~s}]$ & $a_{k}[\mathrm{~Pa}]$ & $\eta_{k}[\mathrm{~Pa} . \mathrm{s}]$ \\
\hline & 10.6855 & 0.058352 & 0.0400 \\
\hline & $2 \quad 0.1396$ & 1.664756 & 0.2324 \\
\hline & $\begin{array}{ll}3 & 0.0389\end{array}$ & 14.560411 & 0.5664 \\
\hline & $4 \quad 0.0059$ & 99.152542 & 0.5850 \\
\hline
\end{tabular}

Com esses dados, obtém-se $R e=0.93$ e $W i=1$. Para verificar a convergência da solução numérica, o escoamento no canal foi simulado nas malhas Malha I $(100 \times 10)$, Malha II $(200 \times 20)$ e Malha III $(400 \times 40)$ até o tempo $t=100$ s e espera-se que após esse tempo, o estado estacionário tenha sido alcançado.

Os perfis de $u(y), \tau^{x x}, \tau^{x y}$ e $\tau^{y y}$ nas 3 malhas, avaliados no meio do canal, são mostrados na Fig. 4.4, na qual exibe-se uma região de amplificação para o perfil de velocidade. Para comparação, o perfil Newtoniano da velocidade é apresentado na Fig. 4.4(a)). A Fig. 4.5 exibe o perfil de velocidades $u$ em algumas seções transversais do canal e pode-se verificar que o perfil de velocidade rapidamente se desenvolve para o perfil do modelo KBKZ. Nessas figuras, pode-se constatar que as soluções estão próximas, o que mostra a convergência das soluções numéricas, 
demonstrando que a técnica de discretização utilizando progressão geométrica apresenta bons resultados para fluidos KBKZ multi-módulos.

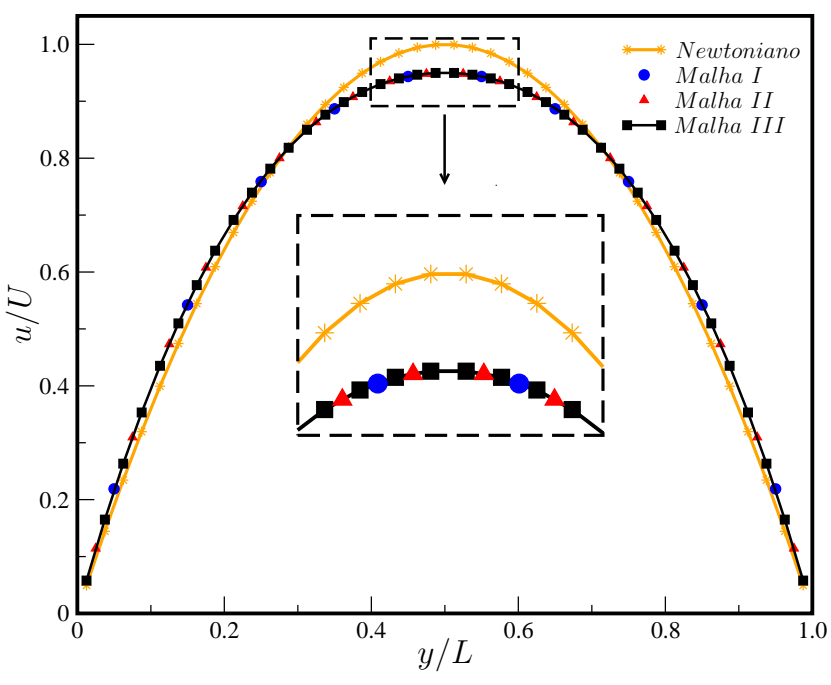

(a)

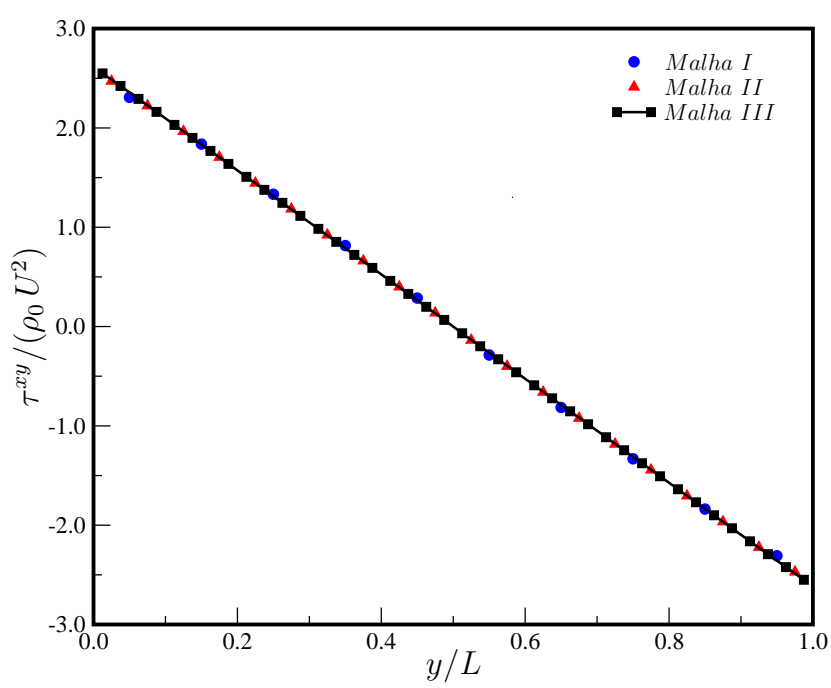

(c)

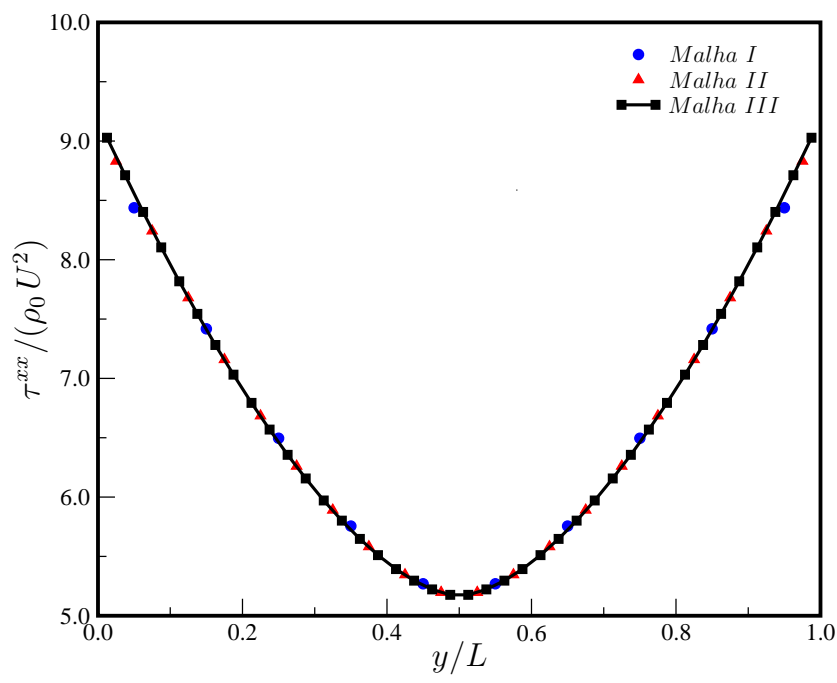

(b)

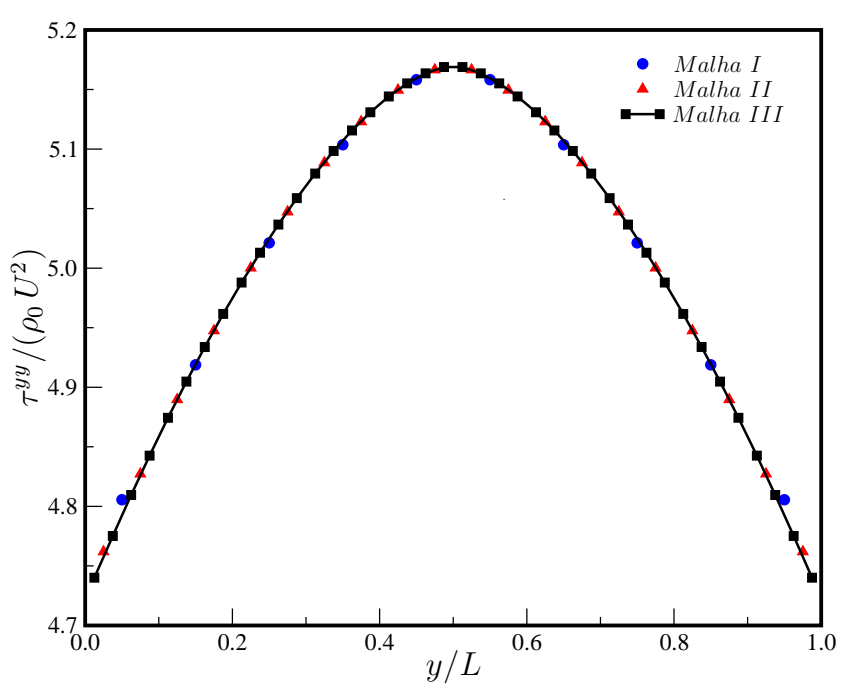

(d)

Figura 4.4: Solução numérica para o Fluido $S 1$ usando as malhas I, II e III. (a) $u(y) / U$; (b) $\tau^{x x}(y) /\left(\rho_{0} U^{2}\right) ;(\mathrm{c}) \tau^{x y}(y) /\left(\rho_{0} U^{2}\right) ;(\mathrm{d}) \tau^{y y}(y) /\left(\rho_{0} U^{2}\right)$. A curva de cor laranja em (a) representa o perfil de velocidades Newtoniano. 


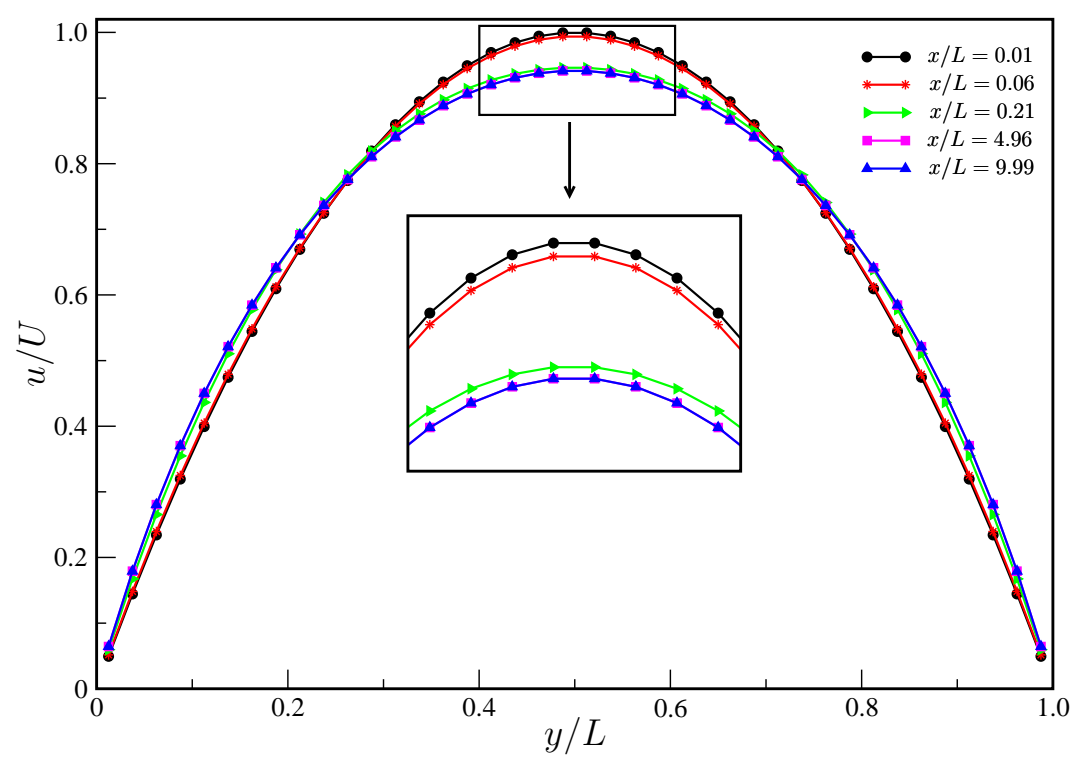

Figura 4.5: Perfil da velocidade $u$ em seções transversais do canal no tempo $t=100 \mathrm{~s}$. Os resultados são apresentados para algumas posições de $x / L$.

\subsection{Escoamentos com superfícies livres}

Nessa seção apresentam-se resultados obtidos da simulação de escoamentos de fluidos KBKZ multi-módulos com superfícies livres em movimento. O problema conhecido como Jet-Buckling é simulado com dois fluidos denominados Fluido B (ver [17]) e Fluido S1 (ver [54]). Um terceiro fluido, conhecido como Fluido M1 (ver [17]) é empregado na simulação do Extrudate-Swell.

\subsubsection{Simulação do Jet-Buckling}

Jatos incidindo sobre placas tem aplicações em muitos processos industriais, como por exemplo, enchimento de recipientes. Este problema têm sido investigado por muitos pesquisadores (ver [56-58]), e sabe-se que após o jato de fluido colidir com a placa alguns fenômenos podem ocorrer, entre eles, o fenômeno conhecido como Jet buckling. Essa é uma instabilidade física na qual, devido à forças viscosas e ondas de compressão, o jato é incapaz de escoar radialmente e acumula-se sobre si próprio, causando a ocorrência do fenômeno Jet-Buckling (ver Fig. 4.6-(a)). 


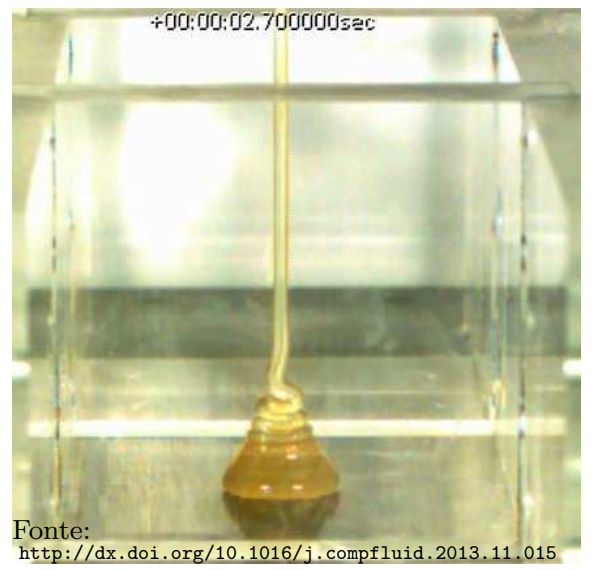

(a)

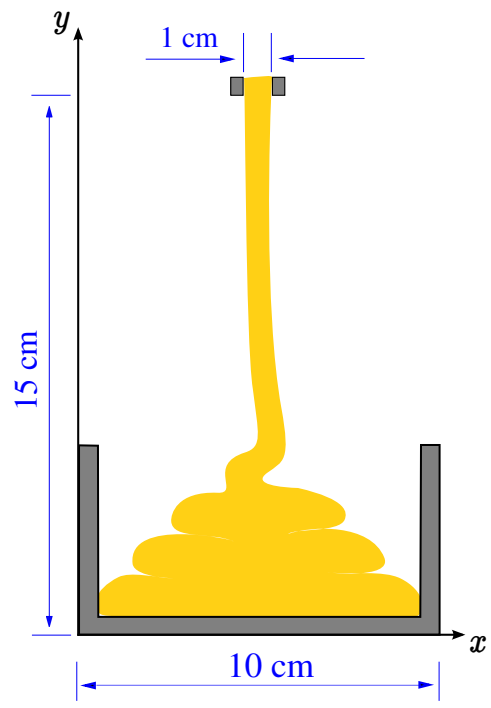

(b)

Figura 4.6: (a) Exemplo do problema Jet-Buckling para um fluido Newtoniano [61]; (b) Descrição do domínio usado na simulação do jato incidente sobre uma parede rígida.

Este problema foi estudado experimentalmente e teoricamente por Cruickshank e Munson [56] com fluidos Newtonianos. Nesse estudo, derivou-se condições baseadas no diâmetro do jato $(D)$, na distância do jato à placa $(H)$ e no número de Reynolds $(R e)$ para prever a ocorrência do fenômeno. Em suas análises, Cruickshank e Munson verificaram que, em duas dimensões, o fenômeno Jet-Buckling ocorre, se as seguintes condições forem satisfeitas

$$
R e<0.56 \text { e } H / D>10 .
$$

Estas condições foram verificadas por muitos pesquisadores que simularam este problema em duas dimensões usando fluidos Newtonianos e Não Newtonianos governados por modelos diferenciais (ver $[7,9,13,52,59,60]$ ). Porém, a simulação desse problema, que envolve superfícies livres interagindo com superfície rígida, utilizando modelos integrais ainda não foi demonstrada. Nesse trabalho, apresentam-se resultados para este problema, usando os fluidos multi-módulos KBKZ, conhecidos como Fluido B (ver tabela 4.5) e Fluido S1 (ver tabela 4.4) . 
Tabela 4.5: Parâmetros do Fluido B usados no problema do jato incidente sobre placa paralela. (ver Mitsoulis [17]).

\begin{tabular}{|c|c|c|c|}
\hline \multicolumn{4}{|c|}{ FLUIDO B } \\
\hline \multicolumn{4}{|c|}{$\rho_{0}=880 \mathrm{~kg} / \mathrm{m}^{3}, \alpha=25286, \lambda_{r e f}=0.14 \mathrm{~s}, \beta=0.1, \eta_{0}=9.79$ Pa.s } \\
\hline$k$ & $\lambda_{k}[\mathrm{~s}]$ & $a_{k}[\mathrm{~Pa}]$ & $\eta_{k}[$ Pa.s $]$ \\
\hline 1 & $1.12 \times 10^{-4} \mathrm{~S}$ & $6.67 \times 10^{4} \mathrm{~Pa}$ & 7.470 Pa.s \\
\hline 2 & $4.12 \times 10^{-2} \mathrm{~S}$ & $1.83 \times 10^{1} \mathrm{~Pa}$ & 0.754 Pa.s \\
\hline 3 & $8.64 \times 10^{-1} \mathrm{~s}$ & $1.83 \times 10^{0} \mathrm{~Pa}$ & 1.581 Pa.s \\
\hline
\end{tabular}

Para mostrar a convergência do método numérico na simulação de escoamentos dependentes do tempo com superfícies livres, o problema do jato foi simulado em várias malhas. O domínio computacional utilizado teve dimensões $10 \mathrm{~cm} \times 15 \mathrm{~cm}$ como ilustra a Fig. 4.6-(b)) e empregou-se um jato de diâmetro $D=1 \mathrm{~cm}$. As malhas utilizadas foram: M1: $40 \times 60(\delta x=D / 4)$; M2: $80 \times 120(\delta x=D / 8) ; \mathrm{M} 3: 100 \times 150(\delta x=D / 10) ; \mathrm{M} 4: 200 \times 300(\delta x=D / 20) ; \mathrm{M} 5: 300 \times 450$ $(\delta x=D / 30)$ e M6: $400 \times 600(\delta x=D / 40)$. Nessas simulações, usou-se $U=0.5 \mathrm{~m} / \mathrm{s}, R e=0.45$, $W i=7, F r=1.59, H / D=15$ e os parâmetros reológicos descritos na tabela 4.5. Nesse estudo, as condições iniciais foram $u(\mathbf{x})=\mathbf{0}, v(\mathbf{x})=\mathbf{U}, \tau^{x x}(\mathbf{x})=\tau^{\mathbf{x y}}(\mathbf{x})=\tau^{\mathbf{y} \mathbf{y}}(\mathbf{x})=\mathbf{0}$. Os resultados obtidos nestas simulações estão apresentados na Fig. 4.7 que exibe a visualização do escoamento em 3 tempos. Analisando esta figura, pode-se verificar que os perfis da superfície livre obtidos em todas as malhas estão próximos. Em particular, nas malhas M3, M4, M5 e M6 os perfis de velocidade estão muito próximos, sendo difícil distingui-los. Para melhor visualização, uma região do domínio foi ampliada. Estes resultados sugerem que o método numérico descrito no capítulo 3 é capaz de simular escoamentos com superfícies livres em movimento.

Para mostrar que esse problema pode ser simulado com modelos integrais, foram realizadas três simulações utilizando o fluido B. O domínio computacional utilizado é mostrado na Fig. 4.6-(b), foi considerada a malha M3 e assumiu-se $N=100$. O fluido foi injetado com 3 valores da velocidade sob ação da gravidade $g_{y}=-9.81 \mathrm{~ms}^{-2}$. Os valores da velocidade de injeção $U$ e dos adimensionais $R e, W i, F r$ estão exibidos na tabela 4.6 onde pode-se ver que as condições de Cruickshank e Munson são satisfeitas e assim, espera-se que o jato com fluido Newtoniano 
apresente o fenômeno de "dobras" (Buckling). Com relação ao jato utilizando o Fluido B, uma análise sobre esse problema utilizando equações integrais ainda não foi apresentada, e os resultados obtidos com modelos diferenciais mostram que esse fenômeno ocorre mais rapidamente que o obtido com modelo Newtoniano. Nessas simulações, o perfil de velocidade no inflow é tomado como constante (perfil reto) e o tensor extra tensão $\tau$ é definido como nulo.

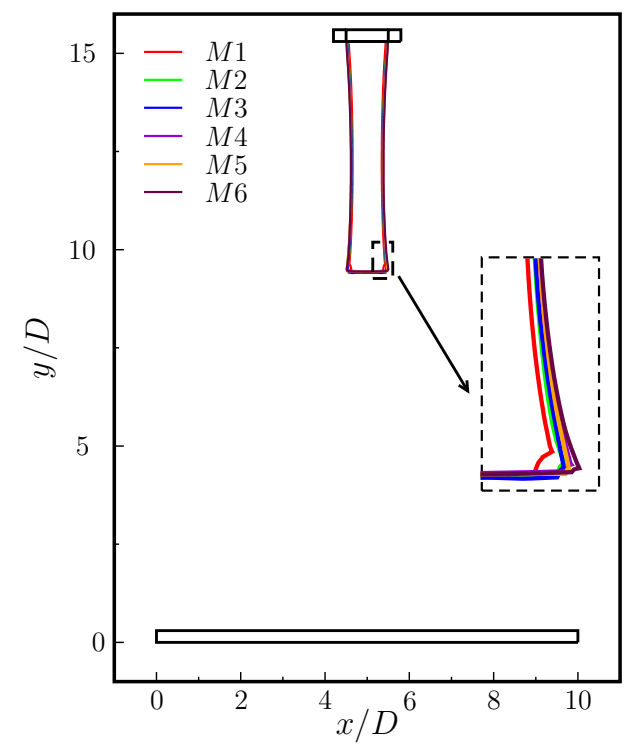

$$
t=0.1 \mathrm{~s}
$$

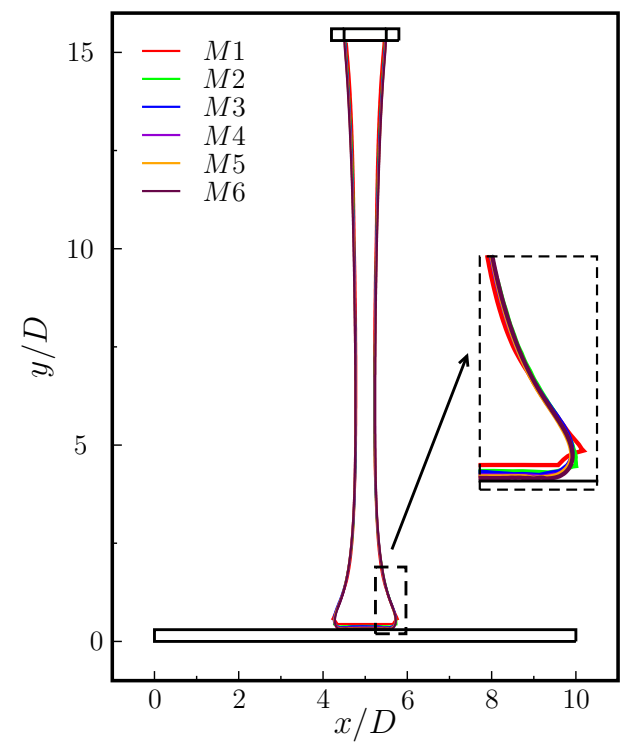

$t=0.2 \mathrm{~s}$

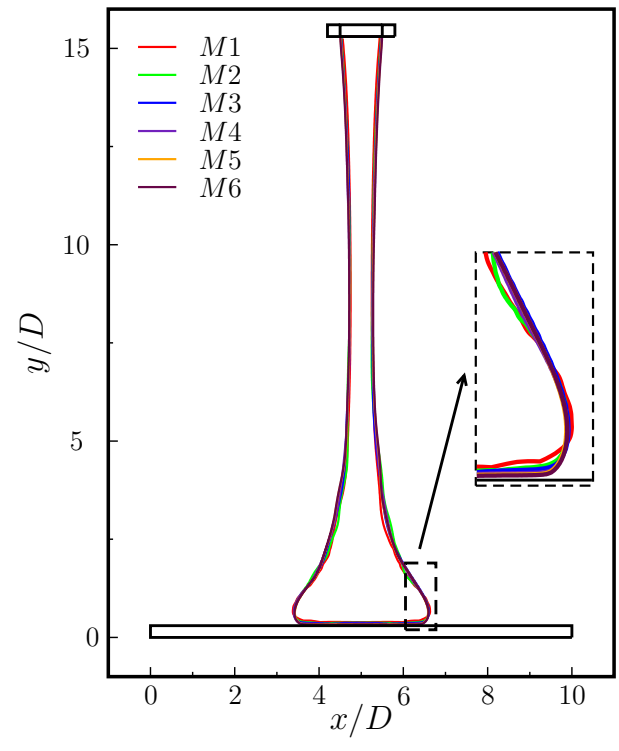

$t=0.3 \mathrm{~s}$

Figura 4.7: Simulação do jato incidindo sobre uma superfície rígida. Visualização do fluido nas malhas M1, M2, M3, M4, M5 e M6. 
Tabela 4.6: Dados usados no problema do jato incidente sobre uma placa paralela.

\begin{tabular}{cccc}
\hline \hline$U\left(\mathrm{~ms}^{-1}\right)$ & $R e$ & $W i$ & $F r$ \\
\hline \hline 0.2 & 0.17 & 2.8 & 0.60 \\
\hline 0.4 & 0.35 & 5.6 & 1.27 \\
\hline 0.6 & 0.53 & 8.4 & 1.91 \\
\hline \hline
\end{tabular}

Os resultados dessas simulações são apresentados nas figuras Fig. 4.8, Fig. 4.9 e Fig. 4.10. Pode-se observar que os resultados com velocidades $U=0.2 \mathrm{~ms}^{-1}$ e $U=0.4 \mathrm{~ms}^{-1}$ (Figs. 4.8 e 4.9 respectivamente) ambos os escoamentos, Newtoniano e viscoelástico, apresentaram o fenômeno do Jet Buckling. Entretanto, com relação aos resultados provenientes da velocidade $U=0.6 \mathrm{~ms}^{-1}$, a Fig. 4.10 mostra que somente o jato utilizando o Fluido $B$ apresentou o fenômeno de "dobras", que acredita-se ser devido a elasticidade do fluido. A análise de Cruickshank e Munson foi feita baseando-se em experimentos e, por isso pode não ser suficientemente precisa, o que pode justificar a não ocorrência das "dobras" no caso do jato Newtoniano com $R e=0.53$, porque esse valor está próximo do valor limitante de $R e=0.56$.

Para confirmar que os resultados obtidos com o Fluido $B$ para $R e=0.53$ foram devido aos efeitos viscoelásticos, foi realizada uma simulação usando o fluido S1, cujos dados estão apresentados na tabela 4.4. Nesta simulação, o domínio utilizado foi $5 \mathrm{~cm} \times 7.2 \mathrm{~cm}$, espaçamento de malha $\delta x=\delta y=1 \mathrm{~mm}, N=250, D=0.6 \mathrm{~cm}, U=0.28 \mathrm{~ms}^{-1}, H / D=12$, o que resulta em $R e=0.94, W i=2.8$ e $F r=1.15$. Para comparação, a correspondente simulação com um jato Newtoniano foi também realizada. Nessa simulação, as condições apresentadas por Cruickshank e Munson não são satisfeitas $(R e=0.94>0.56)$ e portanto, espera-se que o jato Newtoniano não apresente o fenômeno de "dobras". Os resultados dessas simulações em alguns tempos estão exibidos na Fig. 4.11 e conforme esperado, o jato Newtoniano mostrou-se estável sem apresentar "dobras", enquanto o jato simulado com o fluido S1 mostrou-se instável com ocorrência do fenômeno das "dobras". Estes resultados confirmam que a elasticidade modelada pela equação integral KBKZ pode conduzir um jato que colide sobre uma placa rígida à apresentar o fenômeno Jet Buckling. 

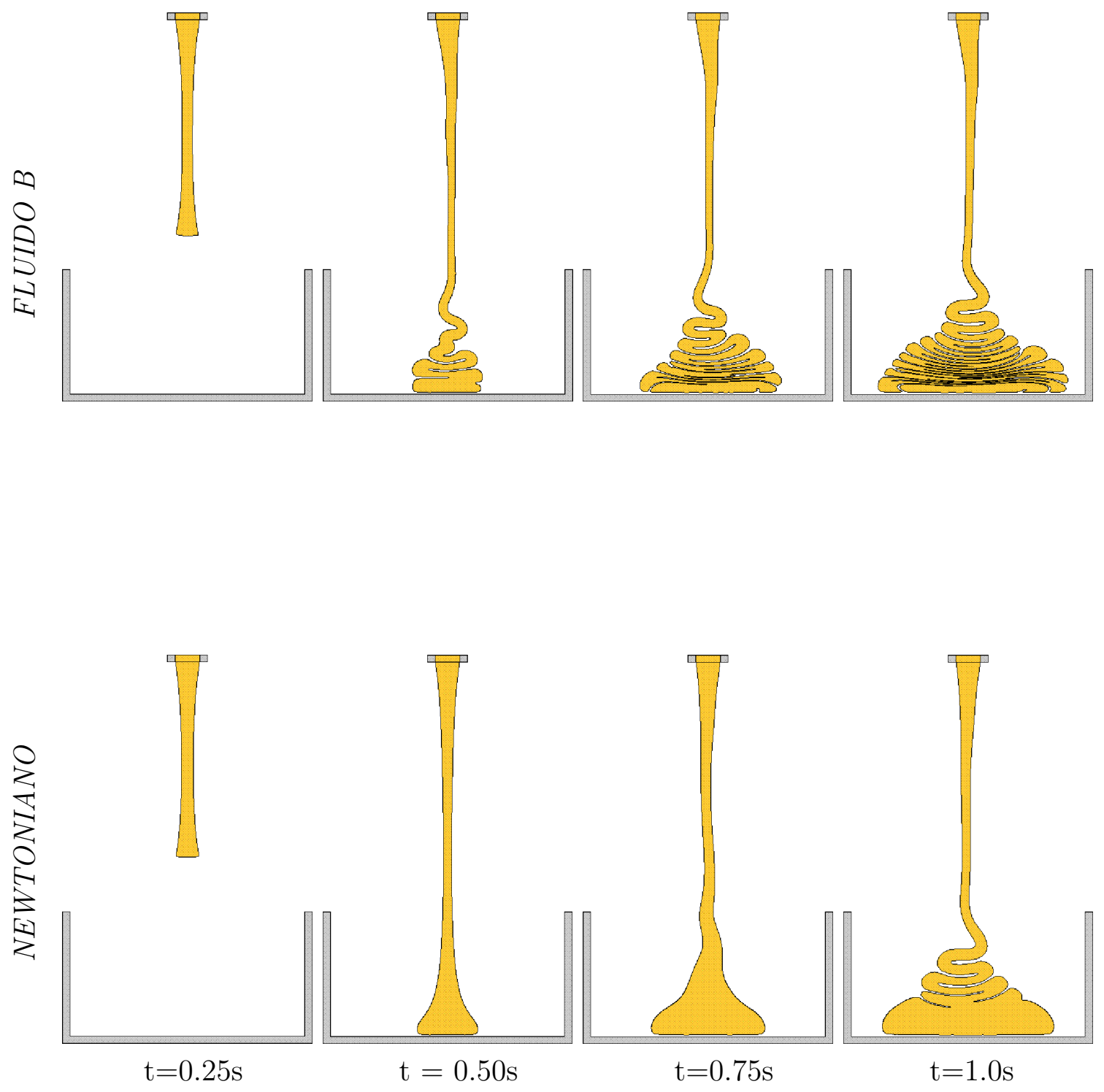

Figura 4.8: Simulação numérica do jato incidente sobre placa rígida utilizando fluido Newtoniano e fluido $B$ com $U=0.2 \mathrm{~ms}^{-1}$. Visualização do escoamento em alguns tempos. 

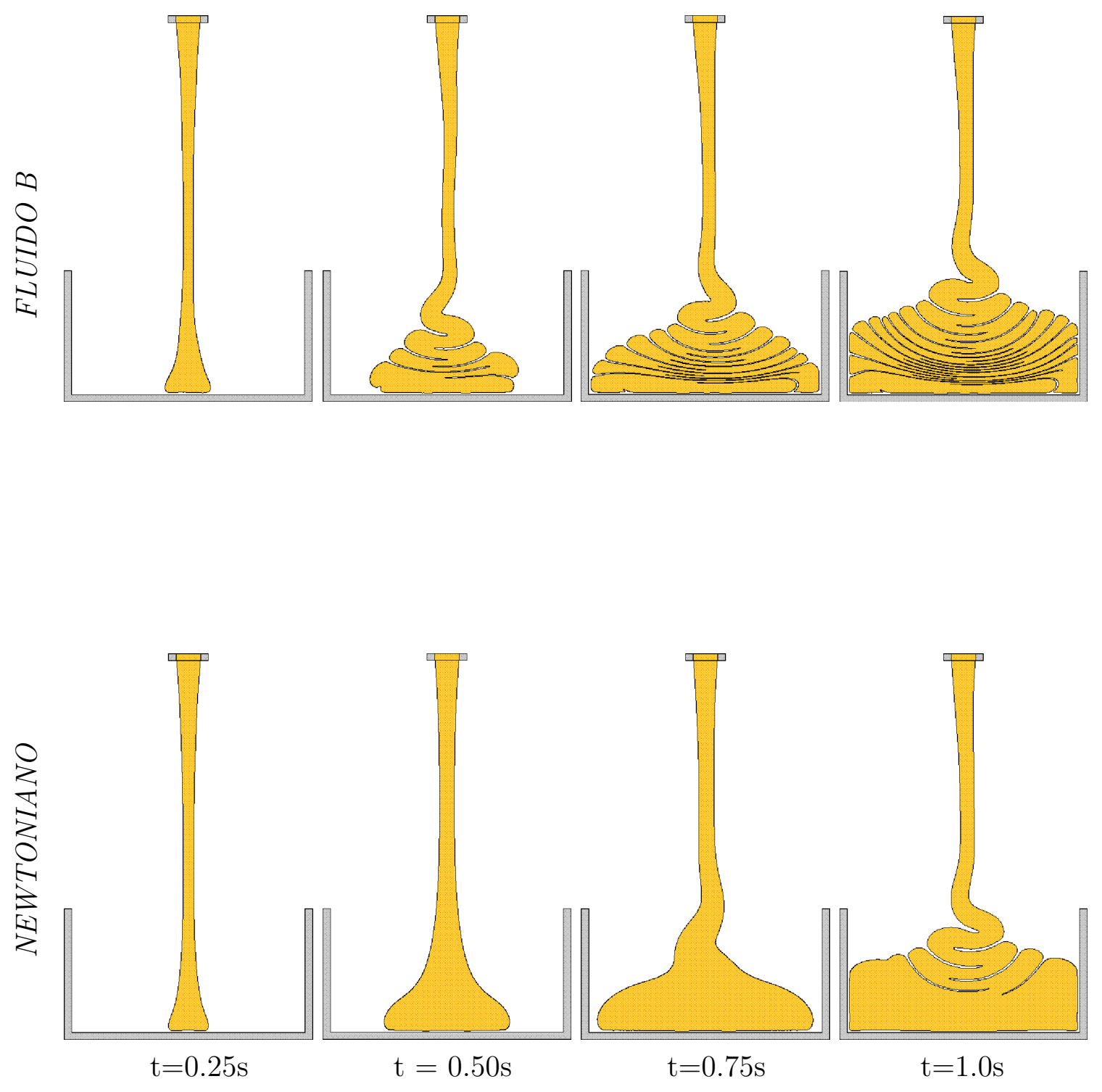

Figura 4.9: Simulação numérica do jato incidente sobre uma placa rígida utilizando fluido Newtoniano e fluido $B$ com $U=0.4 \mathrm{~ms}^{-1}$. Visualização do escoamento em alguns tempos. 

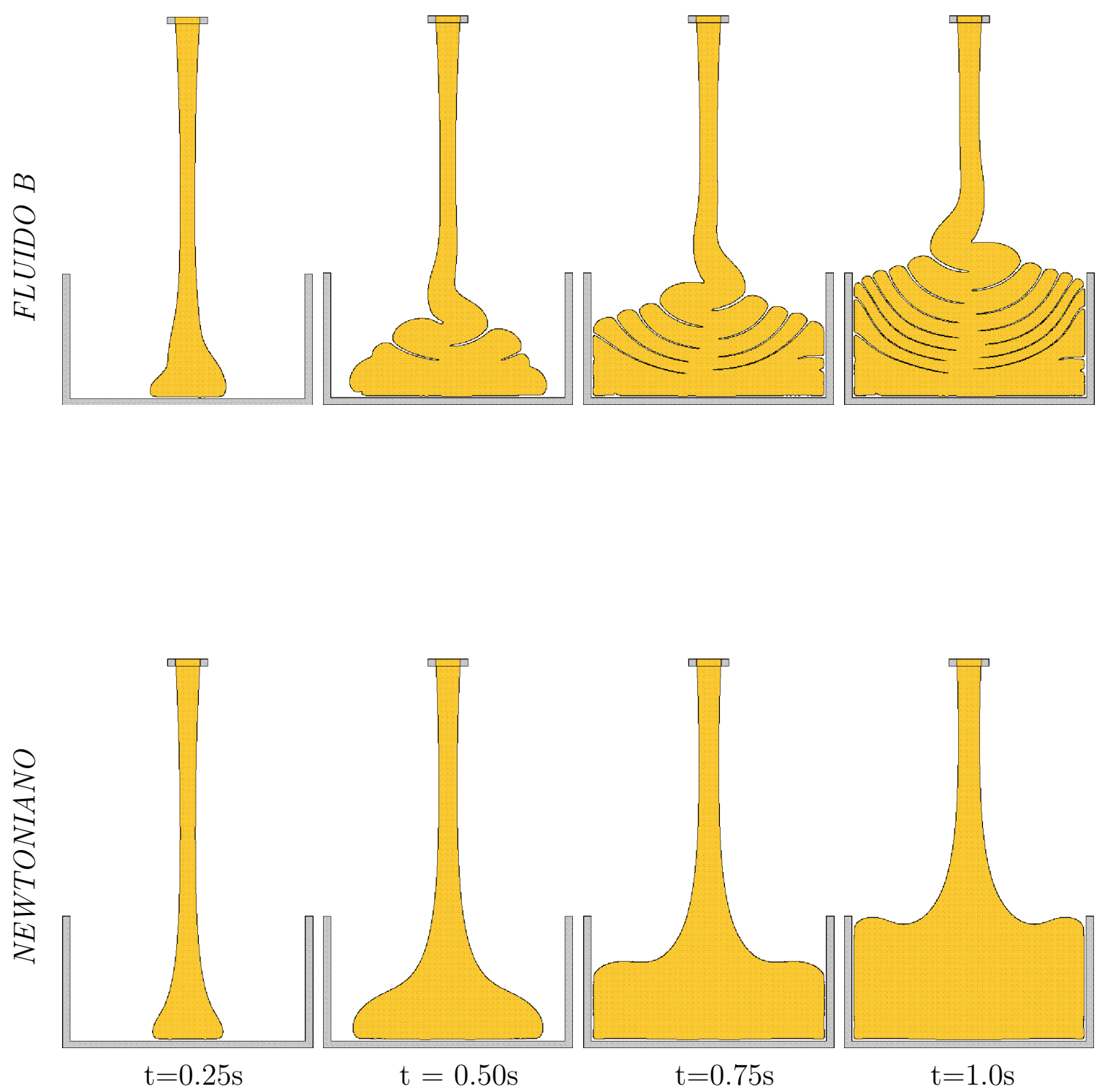

Figura 4.10: Simulação numérica do jato incidente sobre uma placa rígida utilizando fluido Newtoniano e fluido $B$ com $U=0.6 \mathrm{~ms}^{-1}$. Visualização do escoamento em alguns tempos. 

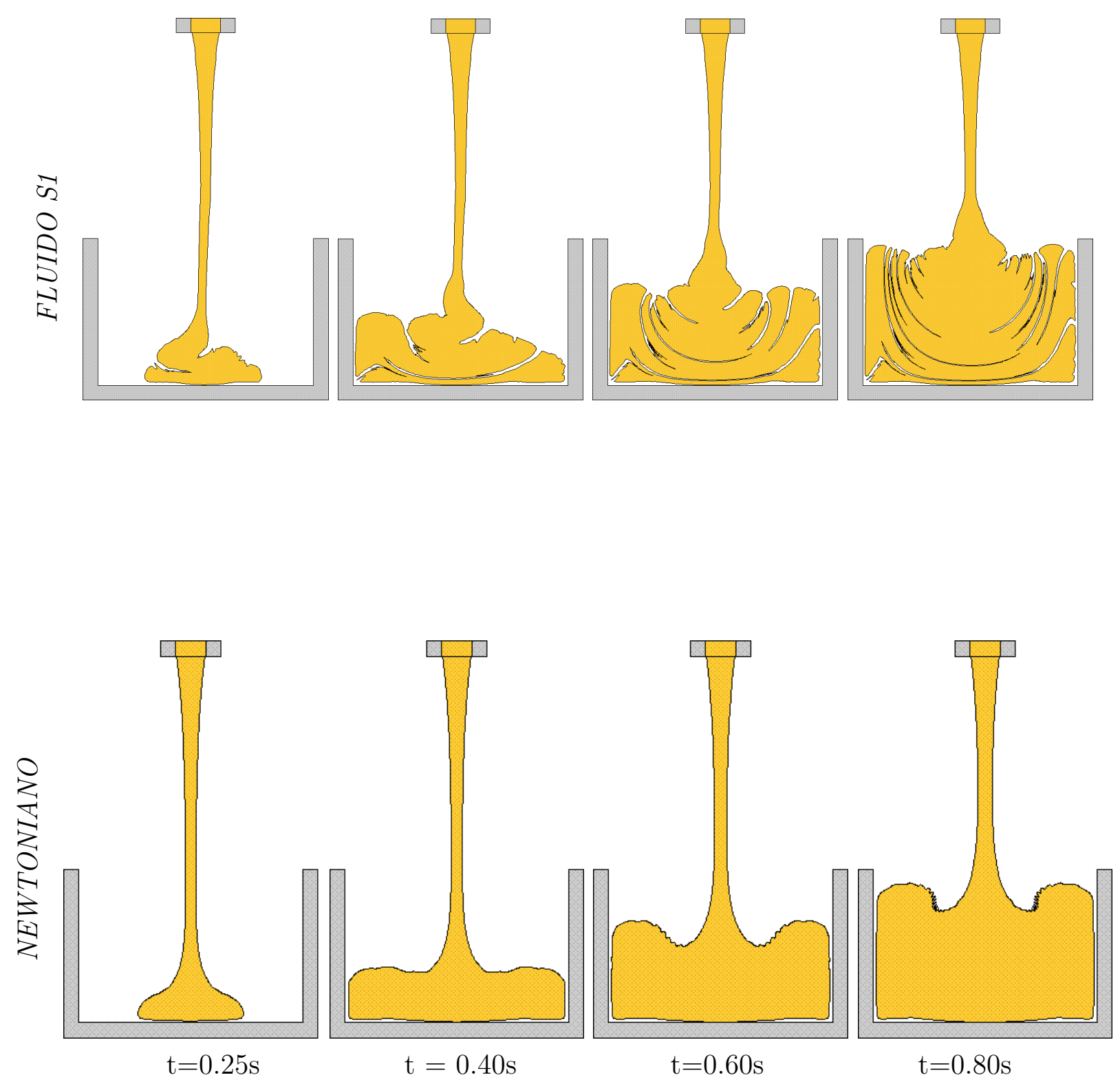

Figura 4.11: Simulação numérica do jato incidente sobre uma placa rígida utilizando fluido Newtoniano e fluido $S 1$ com $U=0.28 \mathrm{~ms}^{-1}$. Visualização do escoamento em alguns tempos. 


\subsubsection{Simulação do Extrudate Swell}

Quando um fluido escoa dentro de um tubo, o mesmo sofre deformações devido à forças de cisalhamento. Por exemplo, durante no processo de extrusão, o polímero experimenta cisalhamento devido a extrusora. Esse efeito provoca o aparecimento de tensões normais e, consequentemente, quando o fluido é expelido na atmosfera o fenômeno do inchamento do extrudado (Extrudate Swell) ocorre (ver Fig. 4.12(a)). Este fenômeno tem atraído a atenção de pesquisadores e simulações numéricas utilizando fluidos do tipo Boger foram inicialmente feitas utilizando-se o modelo Oldroyd-B, que parecia ser o mais apropriado devido às suas características (ver [17]). Os fluidos de Boger são soluções diluídas de polímeros que exibem elevada elasticidade à baixas taxas de cisalhamento, resultando em um grande inchamento do extrudado. Entretanto, os resultados com o modelo Oldroyd-B compararam-se bem com dados experimentais somente em regiões de baixa tensão de cisalhamento e a altas taxas de cisalhamento os resultados não foram satisfatórios. Em altas taxas de cisalhamento, os fluidos de Boger mostram uma tendência em abandonar comportamentos lineares ou quadráticos e assumir um comportamento não linear (ver $[62,63]$ ). O primeiro modelo capaz de simular estes fluidos à altas taxas de cisalhamento foi o modelo integral KBKZ-PSM [23]. Foram realizadas simulações em contrações abruptas e os resultados aproximaram-se bem dos resultados experimentais. Porém, para problemas envolvendo superfícies livres, os resultados para fluidos do tipo Boger não foram muito bons. Simulações de fluidos polietilenos (ver [64-66]) que são fluidos pouco elásticos, apesar de serem não lineares, mostraram-se mais estáveis e mais fáceis de serem realizadas. Alguns pesquisadores exibiram os resultados para o problema do inchamento do extrudado utilizando polietilenos (ver [17]). Considerando fluidos do tipo Boger, o primeiro resultado para o problema do inchamento do extrudado foi apresentado por Trang e Yeow [43] considerando a força da gravidade. Mais recentemente, Mitsoulis [17] exibiu os primeiros resultados deste fenômeno considerando escoamentos com e sem a ação da gravidade, para três tipos de fluidos de Boger utilizando método dos elementos finitos na discretização das equações. Um dos fluidos utilizados por Mitsoulis [17] é o fluido denominado $M 1$ que será utilizado nas simulações apresentadas a seguir. Este fluido foi originalmente caracterizado por Chai e Yeow [18] e contém um espectro com 3 módulos de relaxamento, que estão descritos na tabela 4.7 . 


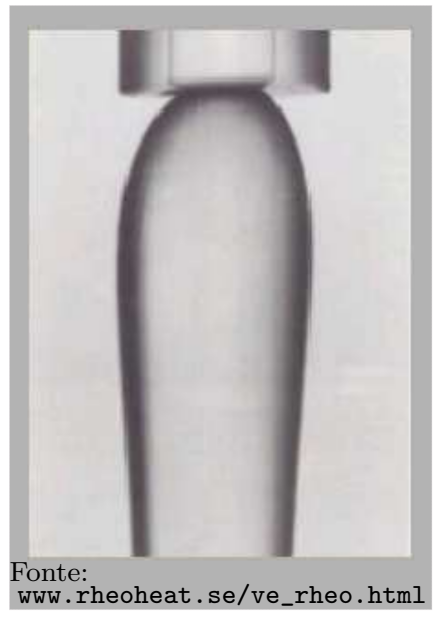

(a)

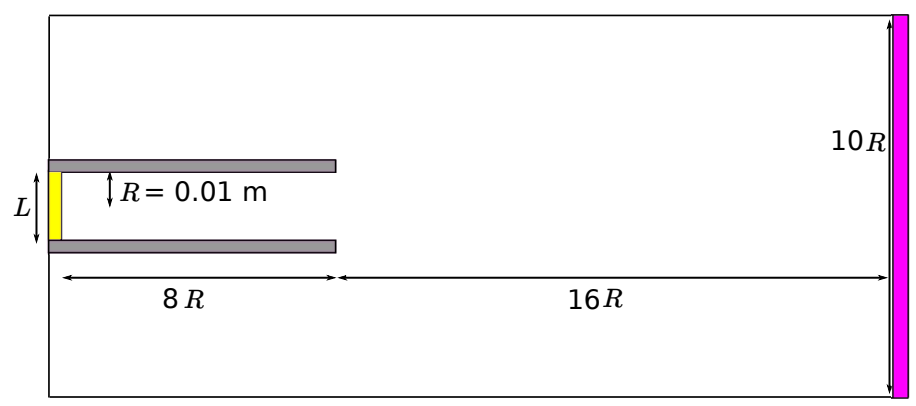

(b)

Figura 4.12: Resultado experimental (a) e ilustração do domínio utilizado no problema do inchamento do extrudado (b).

Tabela 4.7: Parâmetros do Fluido M1 utilizado no problema do inchamento do extrudado. (ver Mitsoulis [17]).

\begin{tabular}{|c|c|c|c|}
\hline \multicolumn{4}{|c|}{ FLUIDO M1 } \\
\hline \multicolumn{4}{|c|}{$\rho_{0}=868 \mathrm{~kg} / \mathrm{m}^{3}, \alpha=34214, \lambda_{r e f}=0.081 \mathrm{~s}, \beta=0.1, \eta_{0}=2.4 \mathrm{~Pa} . \mathrm{s}$} \\
\hline$k$ & $\lambda_{k}[\mathrm{~s}]$ & $a_{k}[\mathrm{~Pa}]$ & $\eta_{k}[\mathrm{~Pa} . \mathrm{s}]$ \\
\hline 1 & $0.4887 \times 10^{-3} \mathrm{~s}$ & $3.1295 \times 10^{3} \mathrm{~Pa}$ & 1.5294 Pa.s \\
\hline 2 & $0.4464 \times 10^{-1} \mathrm{~s}$ & $5.0917 \times 10^{0} \mathrm{~Pa}$ & 0.2273 Pa.s \\
\hline 3 & $2.8384 \times 10^{-1} \mathrm{~s}$ & $2.2783 \times 10^{0} \mathrm{~Pa}$ & 0.6457 Pa.s \\
\hline
\end{tabular}

Para demonstrar que o método numérico desenvolvido nesse trabalho é capaz de simular escoamentos de fluidos com alta elasticidade, o mesmo foi aplicado para simular o inchamento do extrudado do fluido M1. O domínio utilizado é mostrado na Fig. 4.12(b) na qual a região amarela representa uma entrada de fluido ("inflow") e a região rosa determina o local de saída de fluido ("outflow"). As simulações foram realizadas para vários valores da razão de cisalhamento aparente $\dot{\gamma}_{a}$, que é definida em termos da razão volumétrica $Q$ e do tamanho característico do 
canal $L(\operatorname{ver}[17])$ por

$$
\dot{\gamma}_{a}=\frac{3 U}{L}
$$

As simulações iniciaram-se com o canal vazio; na região de entrada do canal o fluido foi injetado com um perfil de velocidade parabólico e as componentes do tensor de Finger foram definidas com a solução exata para escoamentos puramente cisalhantes (ver [53]).

Inicialmente, para verificar independência de malha, utilizou-se $\dot{\gamma}_{a}=16$, com $L=2 R$ e $U=0.106667 \mathrm{~m} / \mathrm{s}$ resultando em $R e=0.771$ e $W i=0.432$ e foram realizadas 5 simulações considerando as malhas $M_{I}: 60 \times 144(\delta x=L / 12) ; M_{I I}: 80 \times 192(\delta x=L / 16) ; M_{I I I}: 100 \times 240$ $(\delta x=L / 20) ; M_{I V}: 120 \times 288(\delta x=L / 24) ; M_{V}: 140 \times 336(\delta x=L / 28)$. Essas simulações foram realizadas até o tempo $t=15$ s onde espera-se que o regime estacionário tenha sido alcançado. A Fig. 4.13 mostra a superfície livre do fluido nas 5 malhas em $t=15$ s e pode observar uma boa concordância das soluções obtidas, mostrando assim a convergência do método numérico para esse problema.

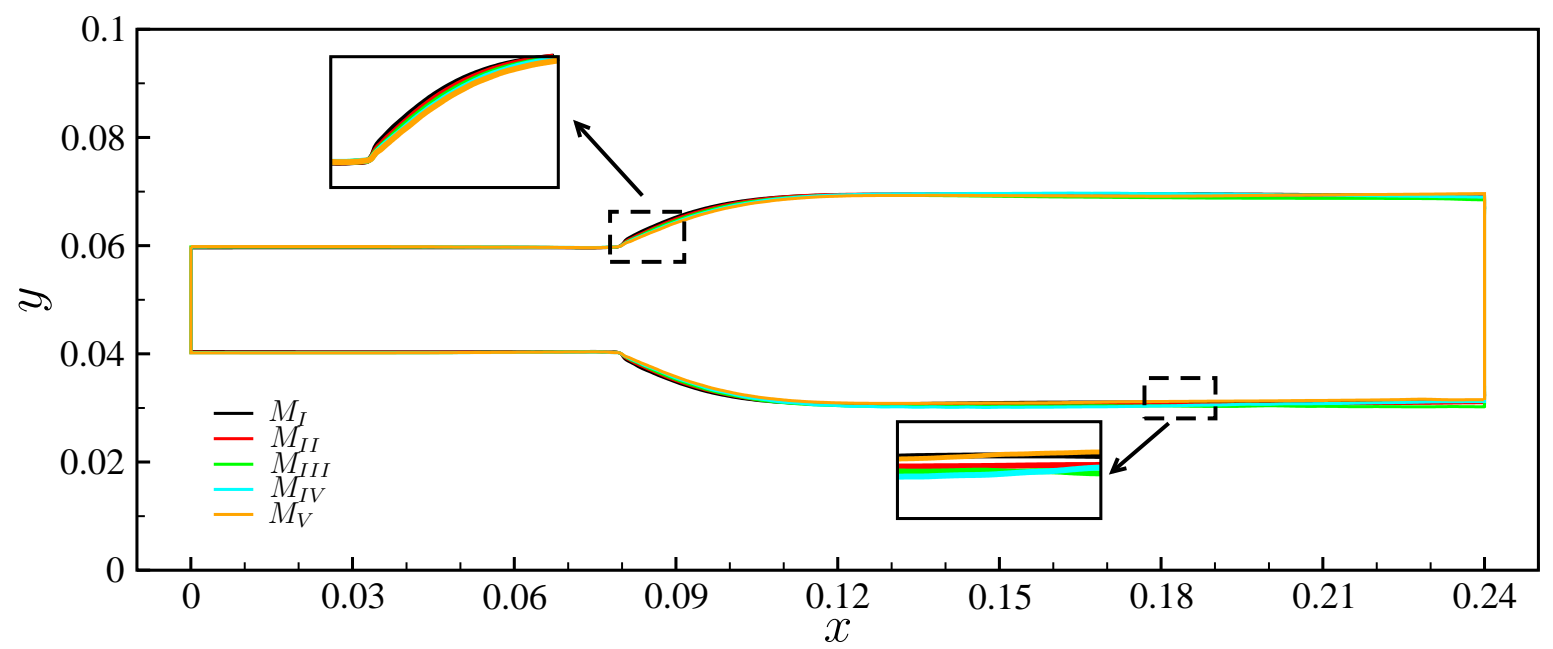

Figura 4.13: Solução numérica no tempo $t=15 \mathrm{~s}$. Superfície do fluido nas malhas $M_{I}, M_{I I}$, $M_{I I I}, M_{I V}$ e $M_{V}$.

A Fig. 4.14 exibe a solução em $t=15$ s para as variáveis $u, v, p$ e a Fig. 4.15 mostra as componentes do tensor extra tensão $\tau$. Esses resultados foram obtidos na malha $M_{I I}$. Pode-se observar que após o inchamento tem-se uma superfície livre horizontal, $u \approx 0.3$ e $v \approx 0$ o que indica que o estado estacionário foi alcançado. 

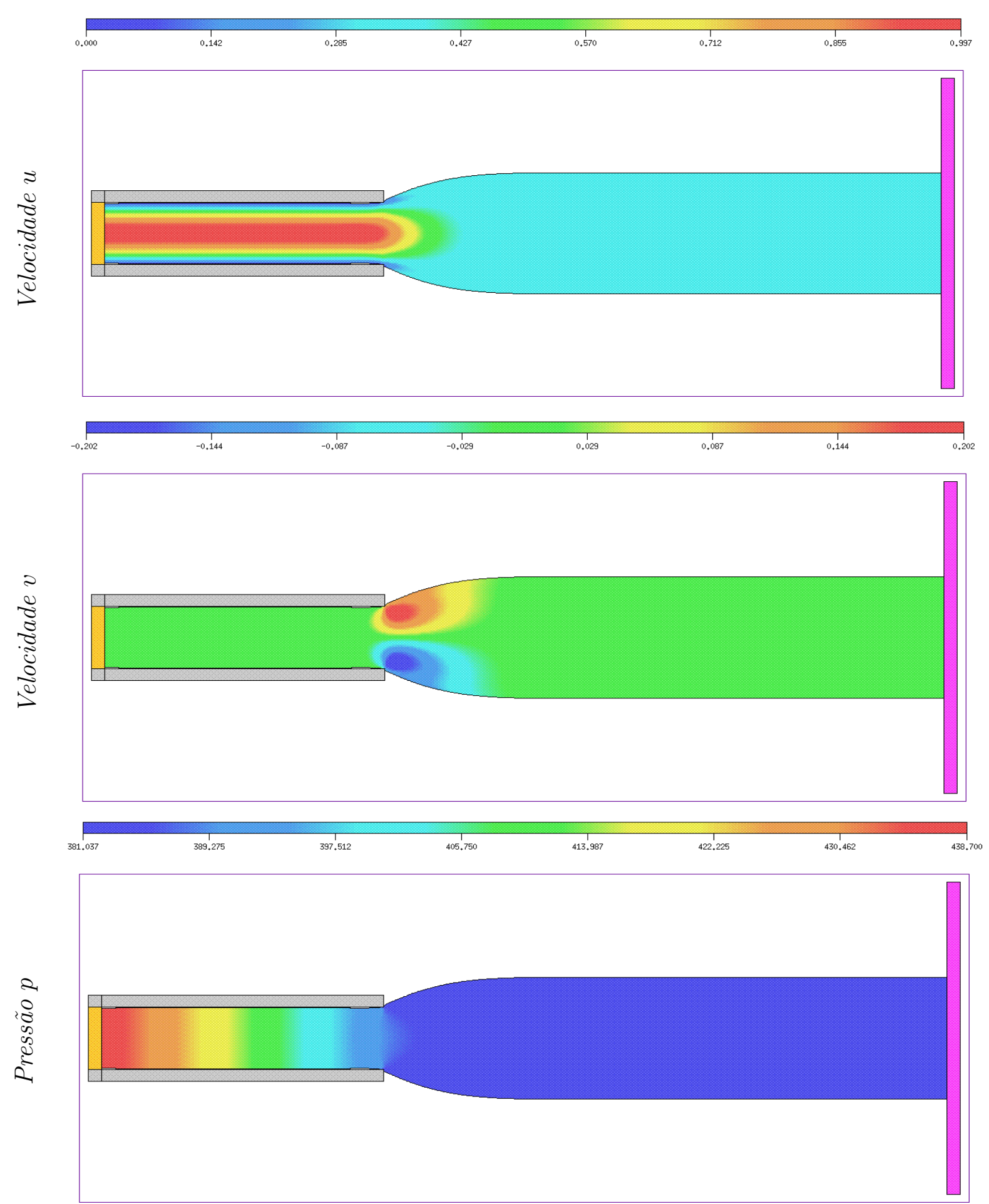

Figura 4.14: Perfil da solução das velocidades nas direções $x, y$ e da pressão para $\dot{\gamma}_{a}=16$ no tempo $t=15 \mathrm{~s}$. 

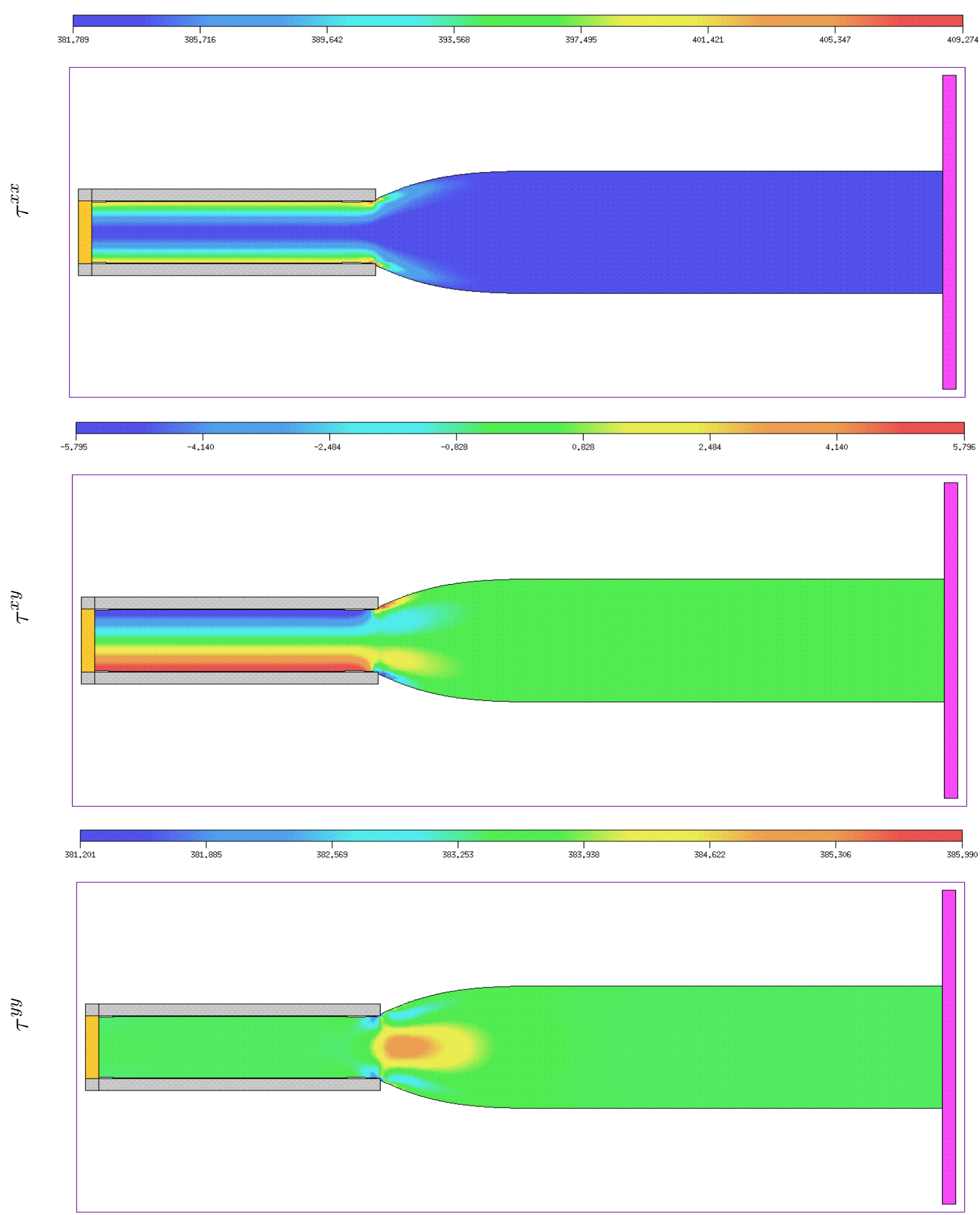

Figura 4.15: Perfil da solução para as componentes do tensor $\tau$ com $\dot{\gamma}_{a}=16$ no tempo $t=15$ s. 
A seguir, utilizando a malha $M_{I I}$ foram realizadas um total de 15 simulações para valores de $\dot{\gamma}_{a}=10+m, m=0,1, \cdots, 14$. Os dados utilizados nestas simulações foram os mesmos empregados no estudo de refinamento de malha (ver Fig. 4.13), e a variação de $\dot{\gamma}_{a}$ gerou os valores de $U, R e, W i$ mostrados na tabela 4.8. Cada simulação foi executada até $t=15 \mathrm{~s}$, e espera-se que nesse tempo o estado estacionário tenha sido atingido.

Tabela 4.8: Dados utilizados na simulação do inchamento do extrudado

\begin{tabular}{cccc}
\hline \hline$\dot{\gamma}_{a}\left(s^{-1}\right)$ & $U\left(m s^{-1}\right)$ & $R e$ & $W i$ \\
\hline \hline 10 & 0.06667 & 0.482 & 0.270 \\
\hline 11 & 0.07334 & 0.530 & 0.297 \\
\hline 12 & 0.08000 & 0.578 & 0.324 \\
\hline 13 & 0.08667 & 0.626 & 0.351 \\
\hline 14 & 0.09334 & 0.675 & 0.378 \\
\hline 15 & 0.10000 & 0.723 & 0.405 \\
\hline 16 & 0.10667 & 0.771 & 0.432 \\
\hline 17 & 0.11334 & 0.819 & 0.459 \\
\hline 18 & 0.12000 & 0.868 & 0.486 \\
\hline 19 & 0.12667 & 0.916 & 0.513 \\
\hline 20 & 0.13334 & 0.964 & 0.540 \\
\hline 21 & 0.14000 & 1.012 & 0.567 \\
\hline 22 & 0.14667 & 1.060 & 0.594 \\
\hline 23 & 0.15334 & 1.109 & 0.621 \\
\hline 24 & 0.16000 & 1.157 & 0.648 \\
\hline \hline
\end{tabular}

A evolução do escoamento para quatro valores de $\dot{\gamma}_{a}$ é mostrada nas figuras Fig. 4.16, Fig. 4.17 e Fig. 4.18 onde pode-se observar que em $t=15$ s todos os jatos escoam pelo outflow e perto do outflow, pode-se ver que as superfícies dos jatos são horizontais e portanto, assume-se que nesse tempo o estado estacionário tenha sido alcançado. 
(a) $t=1.5 \mathrm{~s}$
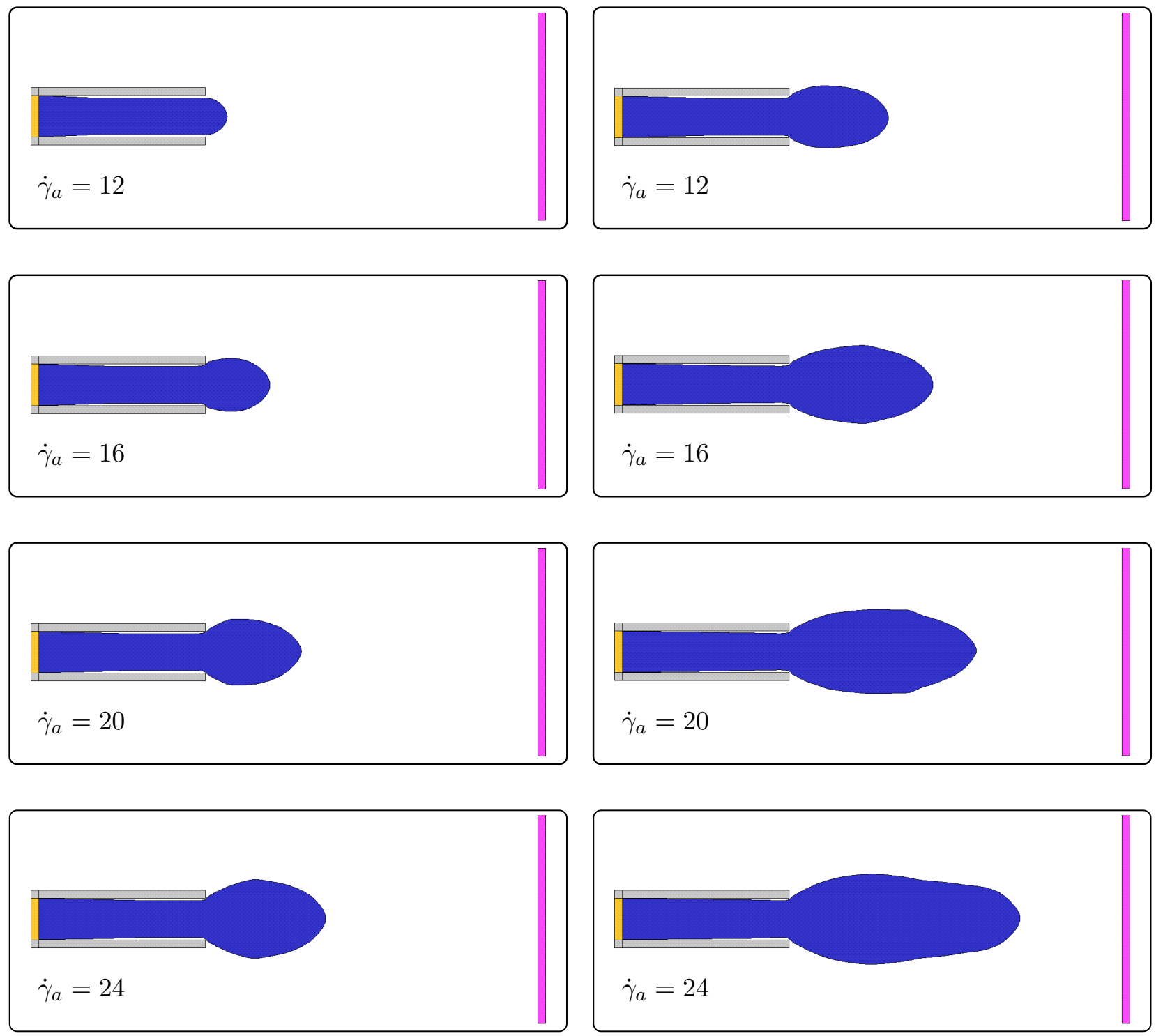

Figura 4.16: Visualização da solução numérica nos tempos $t=1.5 \mathrm{~s}$ e $t=2.5 \mathrm{~s}$ considerando $\dot{\gamma}_{a}=12,16,20,24$. 
(a) $t=3.5 \mathrm{~s}$
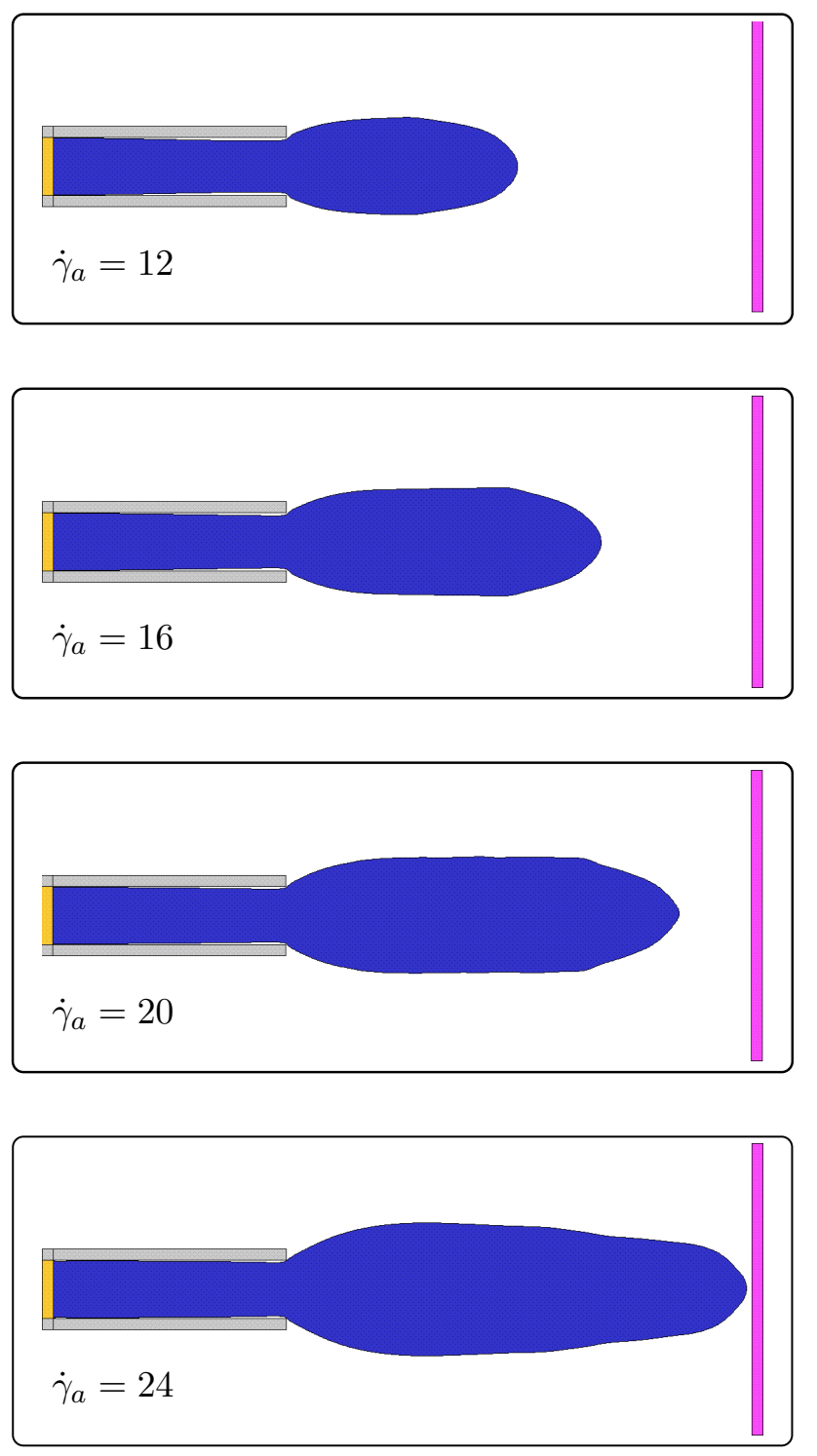

(b) $t=4.5 \mathrm{~s}$
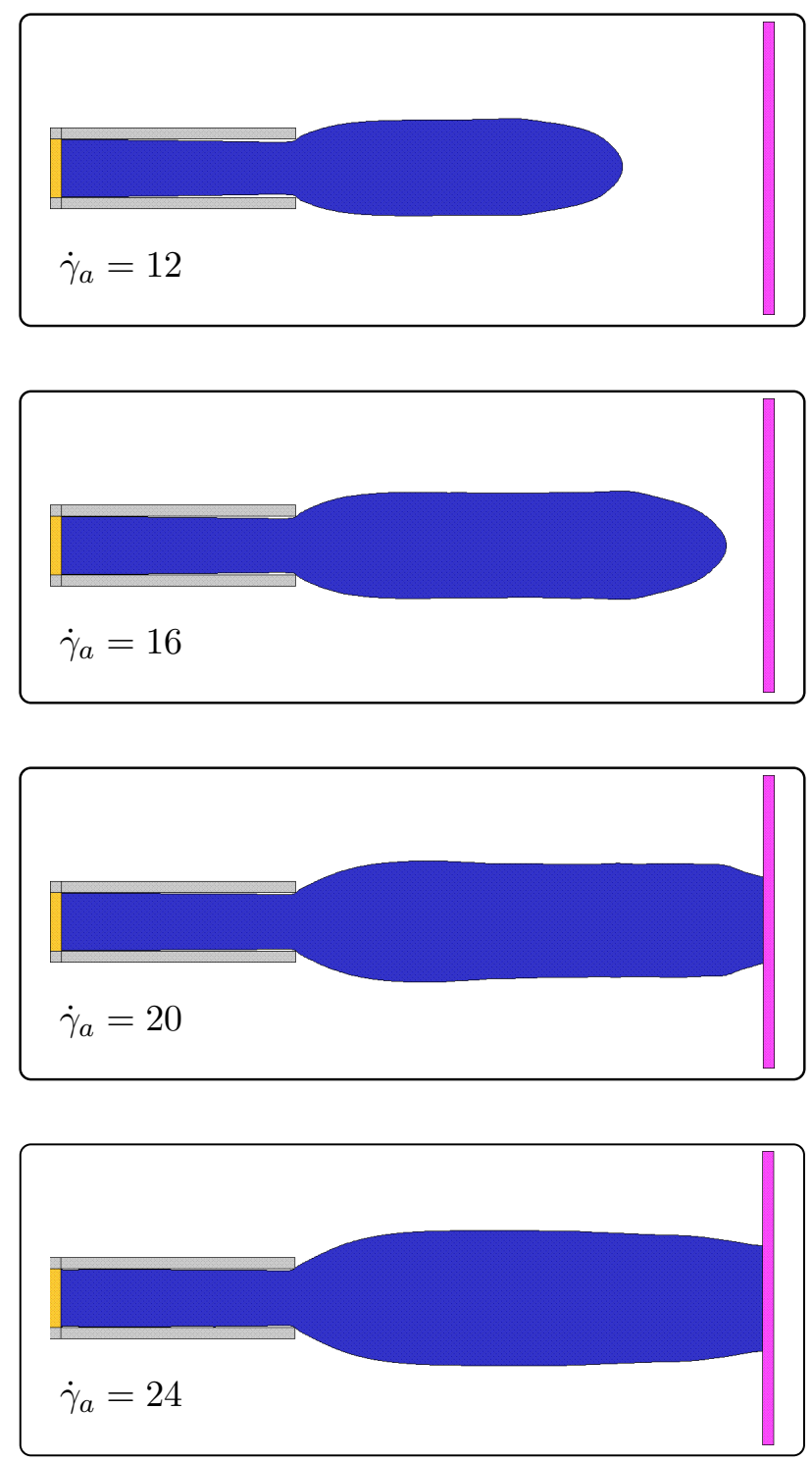

Figura 4.17: Visualização da solução numérica nos tempos $t=3.5 \mathrm{~s}$ e $t=4.5 \mathrm{~s}$ considerando $\dot{\gamma}_{a}=12,16,20,24$. 
(a) $t=6.0 \mathrm{~s}$
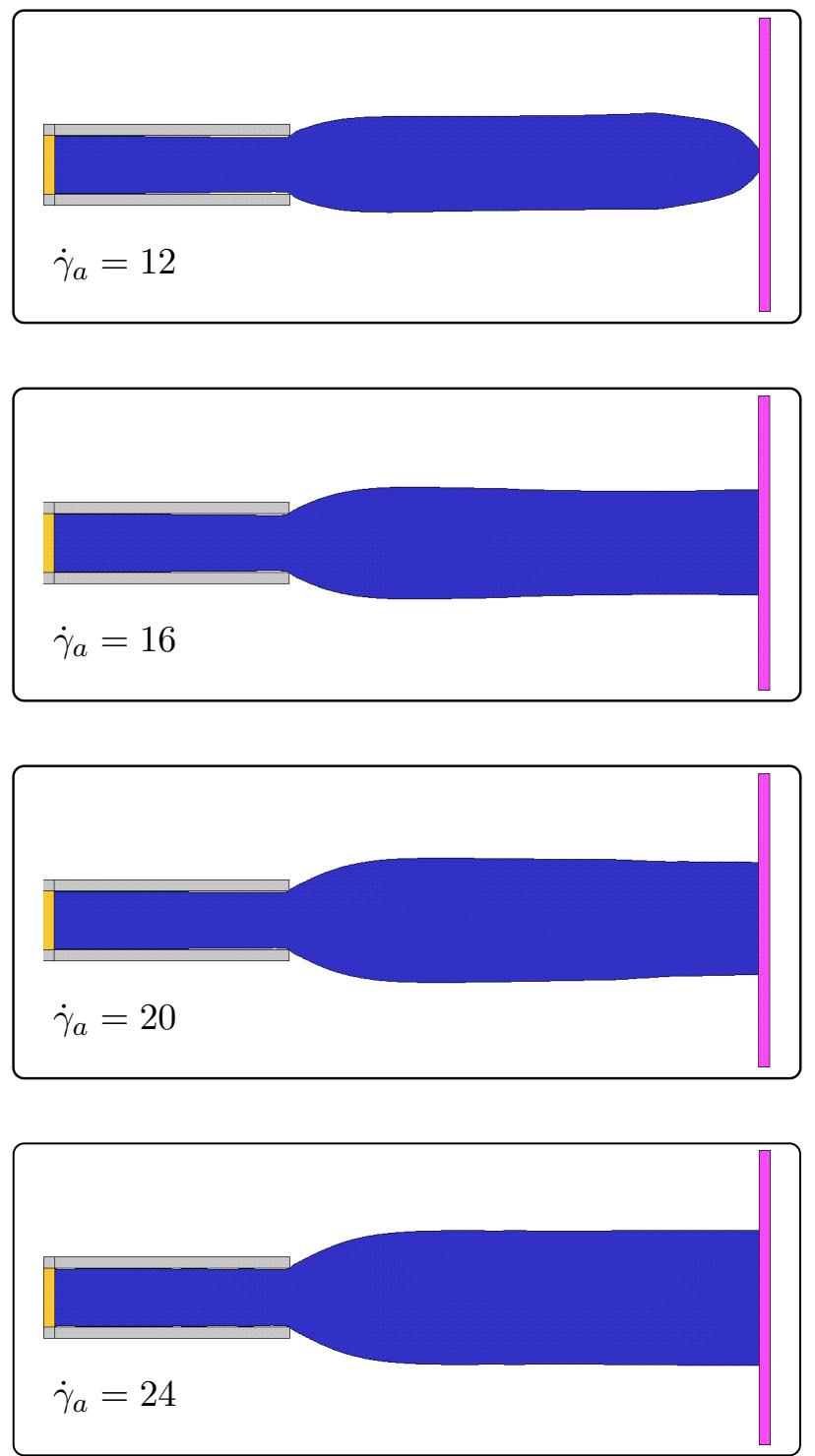

(b) $t=15.0 \mathrm{~s}$
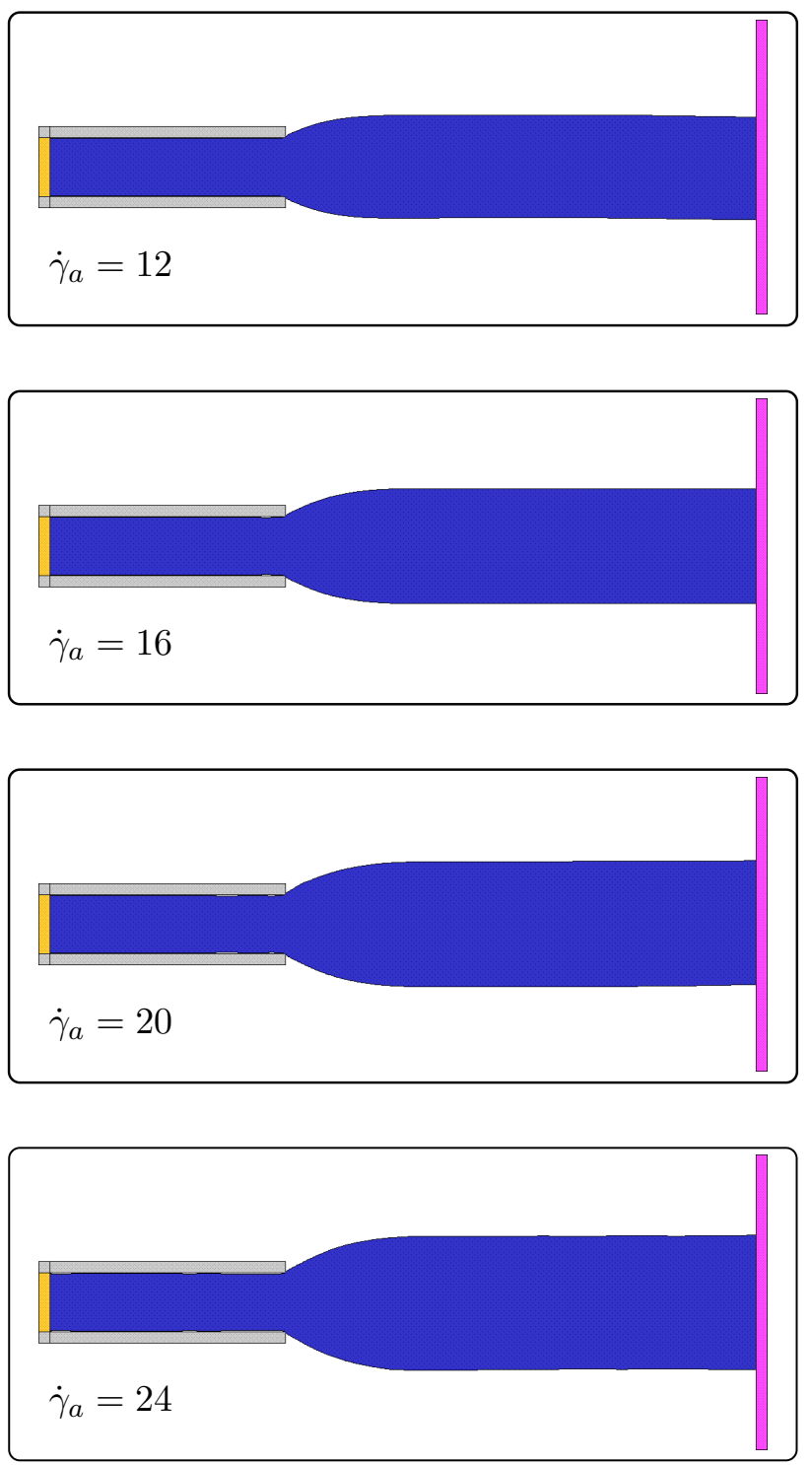

Figura 4.18: Visualização da solução numérica nos tempos $t=6.0 \mathrm{~s}$ e $t=15.0 \mathrm{~s}$ considerando $\dot{\gamma}_{a}=12,16,20,24$. 
A Fig. 4.19 apresenta o perfil de inchamento $\chi$ da superfície livre em função de $\dot{\gamma}_{a}$. Nessa figura, observa-se que o fluido apresenta altas taxas de inchamento o que mostra que o método numérico desenvolvido nesse trabalho é capaz de simular escoamentos de fluidos com alta viscoelasticidade.

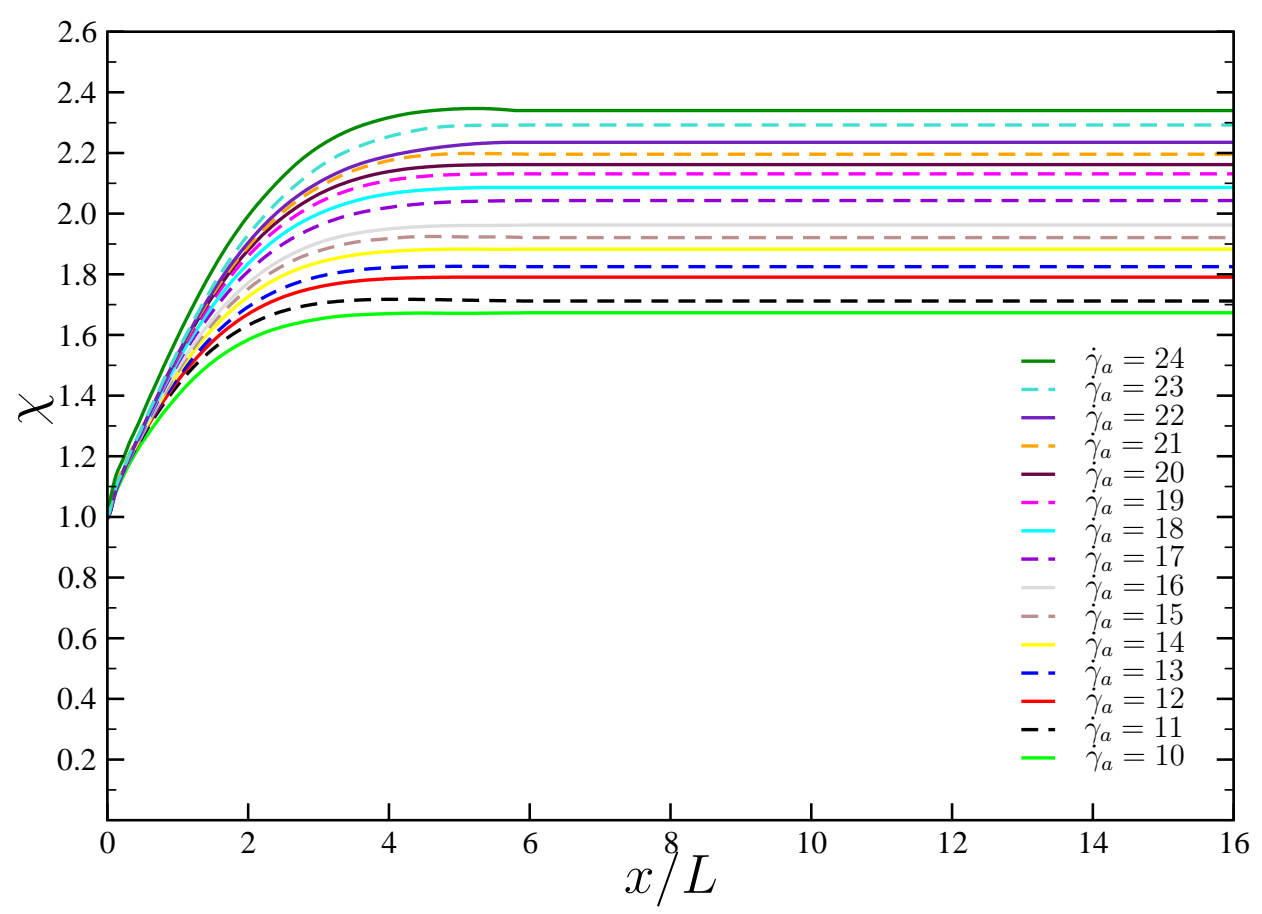

Figura 4.19: Perfil da superfície livre para alguns valores de $\dot{\gamma}_{a}$.

A viscoelasticidade do fluido é medida em termos da razão $S_{r}$, definida por (ver [67])

$$
S_{r}=\frac{N_{1, w}}{2 \tau_{w}^{x y}}=\frac{\left(\tau^{x x}-\tau^{y y}\right)_{w}}{2 \tau_{w}^{x y}}
$$

onde $N_{1, w}$ e $\tau_{w}^{x y}$ são avaliados na parede do canal.

A taxa de inchamento é denotada por $\chi$, definida como

$$
\chi=\frac{D_{\max }}{D}
$$

em que $D_{\max }$ é o diâmetro máximo após o inchamento e $D$ é o diâmetro do canal.

Os valores obtidos para $S_{r}$ e $\chi$ são mostrados na tabela 4.9 , onde vê-se que $0.93 \leq S_{r} \leq 2.22$ e $1.67 \leq \chi \leq 2.34$. Para efeito de comparação, esta tabela apresenta os resultados obtidos por 
Mitsoulis [17] que utilizou o modelo KBKZ-PSM generalizado dado por

$$
\boldsymbol{\tau}(t)=\frac{1}{1-\theta} \int_{-\infty}^{t} M\left(t-t^{\prime}\right) H\left(I_{1}, I_{2}\right)\left(\mathbf{B}_{t^{\prime}}(t) d t^{\prime}+\theta \mathbf{B}^{-1} t^{\prime}(t)\right) d t^{\prime}
$$

No estudo de Mitsoulis [17], adotou-se $\theta=-0.15$.

Tabela 4.9: Valores de $S_{r}$ e $\chi$ em comparação com a solução de Mitsoulis [17]

\begin{tabular}{ccccc}
\hline \hline$\dot{\gamma}_{a}\left(s^{-1}\right)$ & $S_{r}$ & $S_{r}[17]$ & $\chi$ & $\chi[17]$ \\
\hline \hline 10 & 0.93 & --- & 1.67 & --- \\
\hline 11 & 1.02 & --- & 1.71 & --- \\
\hline 12 & 1.116 & 0.97 & 1.79 & 1.76 \\
\hline 13 & 1.20 & --- & 1.82 & --- \\
\hline 14 & 1.30 & --- & 1.88 & --- \\
\hline 15 & 1.39 & 1.21 & 1.92 & 1.94 \\
\hline 16 & 1.48 & --- & 1.96 & --- \\
\hline 17 & 1.57 & --- & 2.04 & --- \\
\hline 18 & 1.66 & 1.45 & 2.09 & 2.17 \\
\hline 19 & 1.75 & --- & 2.13 & --- \\
\hline 20 & 1.84 & --- & 2.16 & --- \\
\hline 21 & 1.93 & 1.68 & 2.19 & 2.35 \\
\hline 22 & 2.02 & --- & 2.23 & --- \\
\hline 23 & 2.13 & --- & 2.29 & --- \\
\hline 24 & 2.22 & --- & 2.34 & --- \\
\hline \hline
\end{tabular}

Para melhor visualização, os resultados são exibidos na Fig. 4.20. Observa-se que os valores obtidos nesse trabalho para $S_{r}$ são superiores aos obtidos por Mitsoulis [17] e os valores de $\chi$ concordam com os resultados de Mitsoulis [17] para baixos valores de $\dot{\gamma}_{a}$. Acredita-se que essas diferenças possam ser atribuídas ao fato que Mitsoulis utilizou um modelo KBKZ com $\theta=-0.15$, que difere do modelo KBKZ-PSM adotado neste trabalho (ver [17]) além de não ser possível determinar quais valores do adimensional $R e$ foram utilizados por Mitsoulis. 


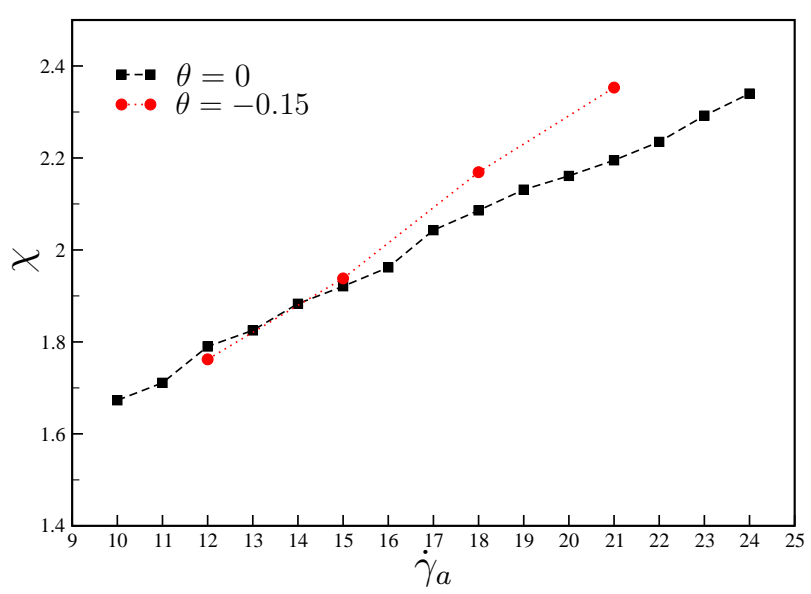

(a)

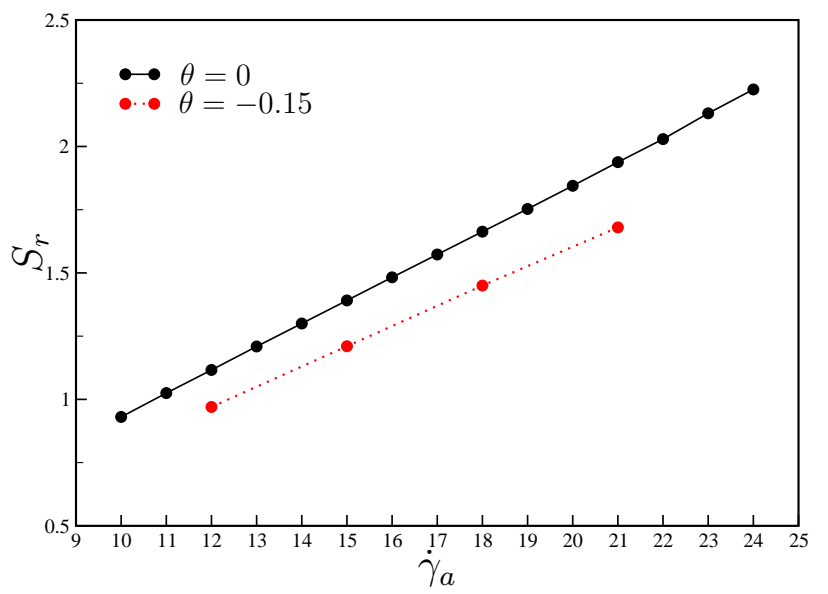

(b)

Figura 4.20: Valores de de $\chi$ (a) e $S_{r}$ (b) $\operatorname{com} \theta=0$ em comparação como os resultados obtidos por Mitsoulis [17] para $\theta=-0.15$.

Na figura 4.20 (a), para valores de $\dot{\gamma}_{a}$ maiores que 15, nota-se que a solução obtida por Mitsoulis [17] apresenta uma taxa de inchamento maior que a obtida neste trabalho. Além da diferença entre os modelos KBKZ-PSM e KBKZ-PSM generalizado, o trabalho de Mitsoulis [17] apresenta uma adimensionalização específica para o adimensional Re. No seu trabalho, Mitsoulis [17] define $R e^{*}=R e / 2$. Para notar a influência desta adimensionalização específica, um teste numerico foi realizado, assumindo $\dot{\gamma}_{a}=21$ ( ver tabela 4.9).

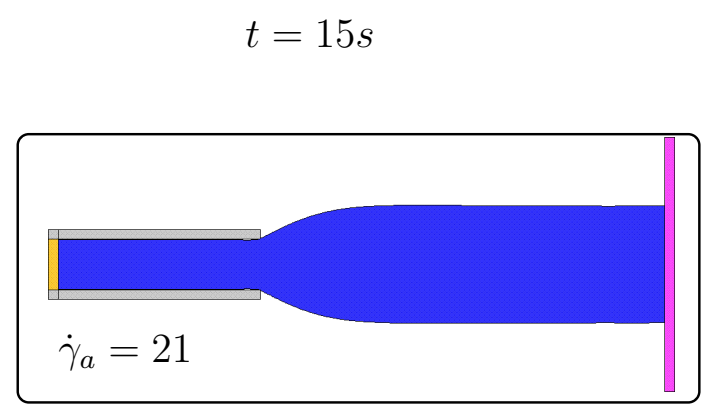

(a)

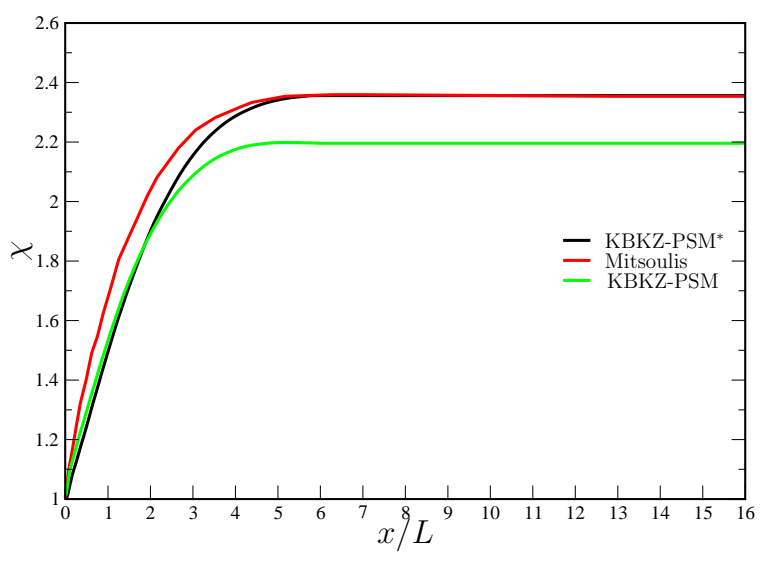

(b)

Figura 4.21: (a)Solução numérica para $\dot{\gamma}_{a}=21$ utilizando $R e^{*}$; (b) inchamento $\chi$ utilizando $R e$ e $R e^{*}$ em comparação com a solução de Mitsoulis [17]. 
Na figura 4.21 (a) exibe-se o perfil da solução em $t=15 \mathrm{~s}$ e em 4.21 (b) apresenta-se a solução para o inchamento $\chi$, considerando o modelo KBKZ-PSM generalizado (Mitsoulis [17]) e considerando o modelo KBKZ-PSM utilizando $R e$ e $R e^{*}$. Nota-se que a mudança específica na adimensionalização de $R e^{*}$ (para mais detalhes ver [17]) influencia a taxa de inchamento $\chi$. Na tabela 4.10 é apresentada a comparação entre os valores $\chi$ e $S r$ considerando a modificação na adimensionalização de $R e$ apresentada por Mitsoulis [17]. Nota-se que com $R e^{*}$ o valor de $S r$ não sofreu grandes variações em relação à adimensionalização inicial de $R e$ (ver seção 2.1), porém, considerando $\chi$, o resultado para $R e^{*}$ se aproximou do resultado obtido por Mitsoulis $[17]$.

Tabela 4.10: Valores de $S_{r}$ e $\chi$ considerando $R e$ e $R e^{*}$ em comparação com a solução de Mitsoulis [17]

\begin{tabular}{ccc}
\hline \hline & $\chi$ & $S_{r}$ \\
\hline Mitsoulis & 2.35384 & 1.68 \\
\hline KBKZ-PSM $^{*}\left(R e^{*}\right)$ & 2.35612 & 1.916 \\
\hline KBKZ-PSM & 2.19856 & 1.927 \\
\hline \hline
\end{tabular}




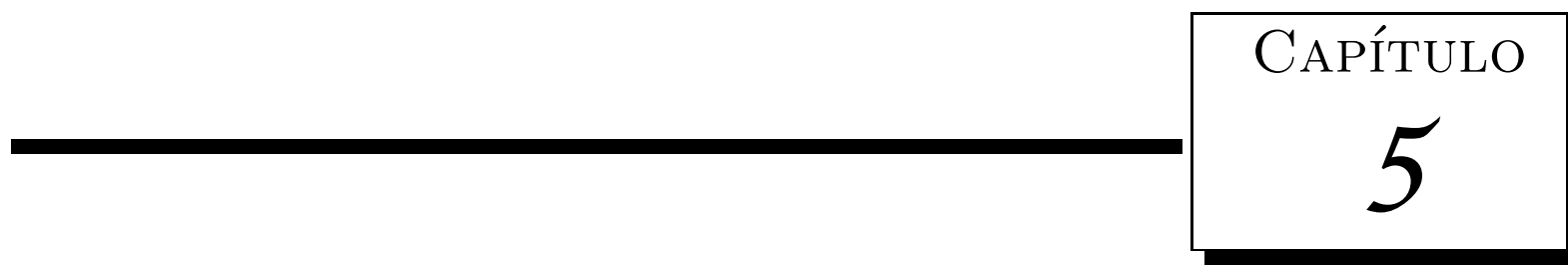

\section{Conclusões}

Esta tese teve como objetivo o desenvolvimento de uma técnica numérica para simular escoamentos viscoelásticos com superfícies livres modelados pela equação constitutiva integral KBKZ-PSM. Este trabalho foi dividido em 5 tópicos. No Capítulo 1, apresentou-se uma descrição dos problemas a serem estudados e dos modelos constitutivos utilizados pelos pesquisadores e o estado da arte da aplicação do modelo KBKZ-PSM a problemas com superfície livre. No Capítulo 2 descreveu-se a formulação matemática e uma descrição detalhada das equações governantes a serem resolvidas; no Capítulo 3 foi discutido em detalhes uma técnica numérica para resolver as equações governantes para escoamentos incompressíveis com superfície livre. O Capítulo 4 contém os resultados obtidos pela aplicação do método numérico, desenvolvido no Capítulo 3, em problemas de verificação do método numérico, jet buckling e inchamento do extrudado.

O método numérico apresentado no Capítulo 3 consiste de uma técnica de diferenças finitas para resolver as equações governantes em que as equações de conservação de quantidade de movimento são resolvidas por um método semi-implícito para obter a solução de $\mathbf{v}^{(n+1)}$ e $p^{(n+1)}$. O tratamento implícito de $\mathbf{v}^{(n+1)}$ e $p^{(n+1)}$ melhorou a estabilidade do método numérico e é uma melhoria com relação ao trabalho anterior apresentado por Tomé e colaboradores [30].

Com relação a solução da equação constitutiva integral, a história do fluido é modelada pelo 
tensor de Finger, $\mathbf{B}_{t_{j}^{\prime}}(\mathbf{x}, t)$, o qual foi obtido pelo método de segunda ordem Euler Modificado e os tempos de deformação $t_{j}^{\prime}$ foram obtidos por uma progressão geométrica que forneceu melhores resultados se comparados com o método da imagem inversa da função memória (ver Tomé e colaboradores [30]). Além dessas melhorias, esse trabalho incluiu um tratamento para escoamentos com superfícies livres que foi o foco principal desse trabalho.

Os resultados de verificação apresentados no Capítulo 4 incluíram a simulação de um escoamento em um canal bidimensional de um fluido KBKZ-PSM com 3-módulos de relaxamento (Fluido B) e também foram obtidos resultados do escoamento de um fluido KBKZ-PSM com 1-módulo de relaxamento e os resultados numéricos foram comparados com a solução analítica que foi desenvolvida nesse trabalho. Em ambas as verificações realizadas, foi empregado refinamento de malha para mostrar a convergência do método numérico. Para mostrar que o método numérico pode tratar de problemas com superfícies altamente deformáveis, o problema conhecido como jet buckling foi simulado utilizando os fluidos Fluido B e $S 1$ e mostrou-se que o fenômeno buckling foi devido aos efeitos de elasticidade do fluido modelados pela equação KBKZ-PSM. Em sequência, foram simulados escoamentos com superfícies livres para fluidos complexos do tipo Boger. Esses fluidos possuem alta elasticidade e são bem aproximados pelo modelo KBKZ-PSM. Resultados numéricos com fluidos de Boger foram apresentados para escoamentos confinados em problemas como contração abrupta e os resultados comparavam bem aos experimentais [68]. No entanto, em escoamentos com superfícies livres, as simulações mostraram-se bem mais complicadas. Os primeiros resultados de escoamentos com superfície livre foram obtidos no problema do inchamento do extrudado e apresentados por Mitsoulis [17] utilizando a técnica de elementos finitos. Nesse trabalho, foram realizadas várias simulações do problema do inchamento do extrudado utilizando o Fluido M1 variando a taxa de cisalhamento aparente $\left(\dot{\gamma}_{a}\right)$ e os resultados concordaram com os obtidos por Mitsoulis [17]. Além disso, os resultados mostraram que para altas taxas de cisalhamento, obteve-se altos valores de inchamento em torno de 140\%, o que demonstra que o método numérico desse trabalho pode tratar de escoamentos com superfície livre de fluidos com alta elasticidade.

Como trabalhos futuros pretende-se estender a metodologia desenvolvida nesse trabalho para escoamentos axissimétricos e tridimensionais. Em escoamentos tridimensionais e com simetria radial, o cálculo do tensor de Finger utilizando o método dos campos de deformação não sofre grandes alterações em relação ao caso bidimensional, a não ser o cálculo de novas variáveis 
devido à dimensão da matriz. Na discretização dos tempos de deformação $t_{j}^{\prime}$, pode-se aplicar a metodologia adotada para escoamentos bidimensionais. Acredita-se assim que a discretização via progressão geométrica apresentará bons resultados em simetria radial e para escoamentos em três dimensões.

Outra linha de pesquisa é a extensão da metodologia à escoamentos não isotérmicos nos quais a equação integral KBKZ-PSM é modificada e resolvida em função do tempo interno do material e não mais em função do tempo $t$ de simulação (ver [69]). 


\section{Referências Bibliográficas}

[1] M. F. Tomé, A. Castelo, A. M. Afonso, M. M. Alves, F. T. Pinho, Application of the log-conformation tensor to three-dimensional time-dependent free surface flows, $J$. Non-Newt. Fluid Mech., 175-176 (2012), 44-54.

[2] M. F. Tomé, R. A. P. Silva, C. M. Oishi, S. McKee, Numerical solution of the upper-convected Maxwell model for three-dimensional free surface flows, Comm. Comput. Phys., 6 (2009), 367-395.

[3] G. Mompean, L. Thais, M. F. Tomé, A. Castelo, Numerical prediction of three-dimensional time-dependent viscoelastic extrudate swell using differential and algebraic models, Comput. Fluids, 44 (2011), 68-78.

[4] M. F. Tomé, A. Castelo, V. G. Ferreira, S. McKee, A finite difference technique for solving the Oldroyd-B model for 3D-unsteady free surface flows, J. Non-Newt. Fluid Mech., 154 (2008), 179-206.

[5] M. Aboubacar, H. Matallah, M. F. Webster, Highly elastic solutions for Oldroyd-B and Phan-Thien Tanner fluids with a finite volume element method: planar contraction flows, J. Non-Newt. Fluid Mech., 103 (2002), 65-103.

[6] J. R. Clermont, M. Normandin, Numerical simulation of extrudate swell for Oldroyd-B fluid using the stream-tube analysis and a streamline approximation, J. Non-Newt. Fluid Mech., 50 (1993), 193-215. 
[7] A. Bonito, M. Picasso, M. Laso, Numerical simulation of 3D viscoelastic flows with free surfaces, J. Comput. Phys., 215 (2006), 691-716.

[8] C. Bérauto, A. Fortin, T. Coupez, Y. Demay, B. Vergnes, J. F. Agassant, A finite element method for computing the flow of multi-mode viscoelastic fluids: comparison with experiments, J. Non-Newt. Fluid Mech., 75 (1998), 1-23.

[9] G. S. Paulo, M. F. Tomé, S. McKee, A marker-and-cell approach to viscoelastic free surface flows using the PTT model, J. Non-Newt. Fluid Mech., 147 (2007), 149-174.

[10] M. F. Tomé, G. S. Paulo, F. T. Pinho, M. A. Alves, Numerical Solution of the PTT constitutive equation for three-dimensional free surface flows, J. Non-Newt. Fluid Mech., 165 (2010), 247-262.

[11] G. S. Paulo, C. M. Oishi, M. F. Tomé, M. A. Alves, F. T. Pinho, Numerical solution of the FENE-CR model in complex flows, J. Non-Newt. Fluid Mech., 204 (2014), 50-61.

[12] C. M. Oishi, F. P. Martins, M. F. Tomé, M. A. Alves, Numerical simulation of drop impact and jet buckling problems using the eXtended Pom Pom model, J. Non-Newt. Fluid Mech., 169-170 (2012), 91-103.

[13] C. M. Oishi, F. P. Martins, M. F. Tomé, J. A. Cuminato, S. McKee, Numerical solution of the eXtended Pom-Pom model for viscoelastic free surface flows, J. Non-Newt. Fluid Mech., 166 (2011), 165-179.

[14] A. Goublomme, B. Draily, M. J. Crochet, Numerical prediction of extrudate swell of a high-density polyethylene, J. Non-Newt. Fluid Mech., 44 (1992), 171-195.

[15] H.J. Park, D.G. Kiriakidis, E. Mitsoulis, BBirefringence studies in die flows of an HDPE melt, J. Rheol., 36 (1992), 1563-1583.

[16] X. L. Luo, E. Mitsoulis, A numerical study of the effect of elongational viscosity on vortex growth in contraction flows of polyethylene melts, J. Rheol., 34 (1990), 309-342.

[17] E. Mitsoulis, Extrudate swell of Boger fluids, J. Non-Newt. Fluid Mech., 165 (2010), $812-824$. 
[18] M. S. Chai, Y. L. Yeow, Modelling of fluid M1 using multiple-relaxation-time constitutive equations, J. Non-Newt. Fluid Mech., 35 (1990), 459-470.

[19] B. Bernstein, E. A. Kearley, L. J. Zapas, A study of stress relaxations with finite strain, Rub. Chem. Tech., 38 (1965), 76-89.

[20] A. Kaye, Non-Newtonian flow in incompressible fluids, Note No. 134, College of Aeronautics, Cranfield, UK, 1962.

[21] K. Osaki, S. Kimura and M. Kurata, Relaxation of shear and normal stresses in double-step shear deformations for a polystyrene solution. A test of the Doi-Edwards theory for polymer rheology, J. Rheol., 25 (1981), 549-562.

[22] R. Larson, The structure and rheology of complex fluids, 1999, Oxford University Press, New York.

[23] A.C. Papanastasiou, L.E. Scriven, C.W. Macosko, An integral constitutive equation for mixed flows: viscoelastic characterization, J. Rheol., 27 (1983), 387-410.

[24] E. Mitsoulis, 50 Years of the K-BKZ constitutive relation for polymers, Polym. Sci., 2013 (2013).

[25] R. I. Tanner, From A to (BK)Z in constitutive relations, J. Rheol., 32 (1988), 673-702.

[26] X. L. Luo, E. Mitsoulis, An efficient algorithm for strain history tracking in finite element computation of non-Newtonian fluids with integral constitutive equations, Int. J. Num. Meth. Fluids, 11 (1990), 1015-1031.

[27] M. Ansari, A. Alabbas, S. G. Hatzikiriakos, E. Mitsoulis, Entry flow of polyethylene melts in tapered dies, Int. Polym. Proc., 25 (2010), 287-296.

[28] E. Mitsoulis, N. A. Malamataris, The free (open) boundary condition with integral constitutive equations, J. Non-Newt. Fluid Mech., 177-178 (2012), 97-108.

[29] P. Olley, R. Spares, P. D. Cotes, A method for implementing time-integral constitutive equations in commercial CFD packages, J. Non-Newt. Fluid Mech., 86 (1999), 337-357. 
[30] M. F. Tomé, M. S. B. Araujo, M. A. Alves, F. T. Pinho, Numerical simulation of viscoelastic flows using integral constitutive equations: A finite difference approach, J. Comput. Phys., 227 (2008), 4207-4243.

[31] X. L. Luo, E. Mitsoulis, Memory phenomena in extrudate swell simulations for annular dies, J. Rheol., 33 (1989), 1307-1327.

[32] A. Goublomme, M. J. Crochet, Numerical prediction of extrudate swell of a high-density polyethylene: further results, J. Non-Newt. Fluid Mech., 47 (1993), 281-287.

[33] V. Ganvir, A. Lele, R. Thaokar, B. P. Gautham, Prediction of extrudate swell in polymer melt extrusion using an arbitrary Langrangian Eulerian (ALE) based finite element method, J. Non-Newt. Fluid Mech., 156 (2009), 21-28.

[34] R. Ahmed, R. F. Liang, M. R. Mackley, The experimental observation and numerical prediction of planar entry flow and die swell for molten polyethylenes, J. Non-Newt. Fluid Mech., 59 (1995), 129-153.

[35] O. Hassager, M. I. Kolte, M. Renardy, Failure and nonfailure of fluid filaments in extension, J. Non-Newt. Fluid Mech., 76 (1998), 137-151.

[36] H. K. Rasmussen, Time-dependent finite-element method for the simulation of three-dimensional viscoelastic flow with integral models, J. Non-Newt. Fluid Mech., 84 (1999), 217-232.

[37] J. M. R. Marín, H. K. Rasmussen, Lagrangian Finite-Element Method for the Simulation of K-BKZ Fluids with Third Order Accuracy, J. Non-Newt. Fluid Mech., 156 (2009), $177-188$.

[38] O. Hassager, J. M. N. Marin, K. Yu, H. K. Rasmunssen, Polymeric liquids in extension: fluid mechanics or rheometry, Rheol. Acta, 49 (2010), 543-554.

[39] G. H. McKinley, T. Sridhar, Filament-stretching rheometry of complex fluids, Annu. Rev. Fluid Mech., 34 (2002), 375-415.

[40] M. I. Kolte, H. K. Rasmussen, O. Hassager, Transient filament stretching rheometer II: Numerical simulation, Rheol. Acta, 36 (1997), 285-302. 
[41] H. K. Rasmussen, O. Hassager, Three-dimensional simulations of viscoelastic instability in polymeric filaments, J. Non-Newt. Fluid Mech., 82 (1999), 189-202.

[42] A. Bach, H. K. Rasmussen, P. Longin, O. Hassager, Growth of non-axisymmetric disturbances of the free surface in the filament stretching rheometer: experiments and simulation, J. Non-Newt. Fluid Mech., 108 (2002), 163-186.

[43] C.T. Trang, Y.L. Yeow, Extrudate swell of Newtonian and non-Newtonian fluids - the effect of gravitational body force, J. Non-Newtonian Fluid Mech., 20 (1986), 103-116.

[44] E. A. J. F. Peters, M. A. Hulsen, and B. H. A. A. van den Brule, Instationary Eulerian viscoelastic flow simulation using time separable Rivlin-Sawyers constitutive equations, $J$. Non-Newt. Fluid Mech., 89 (2000), 209-228.

[45] M. A. Hulsen, E. A. J. F. Peters, B. H. A. A. van den Brule, A new approach to the deformation fields method for solving complex flows using integral constitutive equations, J. Non-Newt. Fluid Mech., 98 (2001), 201-221.

[46] A. A. Amsden, F. H. Harlow, A simplified MAC technique for incompressible fluid flow calculations, J. Comput. Phys., 6 (1970), 332-335.

[47] F. H. Harlow, J. E. Welch, Numerical calculation of time-dependent viscous incompressible flow of fluid with free surface, Phys. Fluids, 8 (1965), 2182-2189.

[48] M. F. Tomé, A. Castelo, J. Murakami, J. A. Cuminato, R. Minghin, M. C. F. Oliveira, S. McKee, Numerical simulation of Axisymmetric free surface flows, J. Comput. Phys., 157 (2000), 441-472.

[49] M. F. Tomé, S. McKee, GENSMAC: A computational marker-and-cell method for free surface flows in general domains, J. Comput. Phys., 110 (1994), 171-186.

[50] G. K. Batchelor, An Introduction of Fluid Dynamics, Cambridge University Press, Cambridge, 1967.

[51] M. Alves, P. Oliveira, F. T. Pinho, A convergent and universally bounded interpolation scheme for the treatment of advection, Int. J. Num. Meth. Fluids, 41 (2003), 47-75. 
[52] M. F. Tomé, B. Duffy, S. McKee, A numerical technique for solving unsteady non-Newtonian free surface flows, J. Non-Newt. Fluid Mech., 62 (1996), 9-34.

[53] M.F. Tomé, J. Bertoco, C.M. Oishi, M.S.B. Araujo, D. Cruz, F.T. Pinho, M. Vynnycky, A finite difference technique for solving a time strain separable K-BKZ constitutive equation for two-dimensional moving free surface flows, J. Comput. Phys., 311 (2016), 114-141.

[54] L. M. Quinzani, G. H. McKinley, R. A. Brown, and R. C. Armstrong, Modeling the rheology of polyisobutylene solutions, J. Rheol., 34 (1990), 705-748.

[55] R. J. LeVeque, Finite difference methods for ordinary and partial differential equations: steady-state and time-dependent problems, Vol. 98, Siam, 2007.

[56] J. O. Cruickshank, B. R. Munson, Viscous fluid buckling of plane and axisymmetric jets, J. Fluid Mech., 113 (1981), 221-239.

[57] M. F. Tomé, S. McKee, L. Barratt, D. A. Jarvis, A. J. Patrick, An experimental and numerical investigation of container filling with viscous liquids, Int. J. Num. Meth. Fluids, 31 (1999), 1333-1351.

[58] N. M. Ribe, A general theory of the dynamics of thin viscous jets, J. Fluid Mech., 457 (2002), 255-283.

[59] M. F. Tomé, A. Castelo, J. A. Cuminato, N. Mangiavacchi, S. McKee. GENSMAC3D: A numerical method for solving unsteady three-dimensional free surface flows, Int. J. Num. Meth. Fluids, 37 (2001), 747-796.

[60] Xiaoyang X, Jie O., Tao J., Qiang Li, Numerical simulation of 3D-unsteady viscoelastic free surface flows by improved smoothed particle hydrodynamics method, J. Non-Newt. Fluid Mech., 177-178 (2012), 109-120.

[61] M. F. Tomé, A. Castelo, M. Nóbrega, O. Carneiro, G. S. Paulo, F.T. Pereira, Numerical and experimental investigations of three-dimensional container filling with Newtonian viscous fluids, Comput. Fluids, 90 (2014), 172-185. 
[62] D.V. Boger, Dilute polymer solutions and their use to model polymer processing flows, Interrelations Between Processing Structure and Properties of Polymeric Materials, Amsterdam (1984), 307-323.

[63] D.V. Boger, Model polymer fluid systems, Pure and App. Chemistry, 57 (1985), 921-930.

[64] X.L. Luo, R.I. Tanner, Finite element simulation of long and short circular die extrusion experiments using integral models, Int. J. Numer. Methods Eng., 25 (1988), 9-22.

[65] X.L. Luo, E. Mitsoulis, Memory phenomena in extrudate swell simulations from annular dies, J. Rheol., 33 (1989), 1307-1327.

[66] G. Barakos, E. Mitsoulis, Numerical simulation of extrusion through orifice dies and prediction of Bagley correction for an IUPAC-LDPE melt, J. Rheol., 39 (1995), 193-209.

[67] R. I. Tanner, Engineering Rheology, Oxford University Press, Oxford, 2000.

[68] H.J. Park, E. Mitsoulis, Numerical simulation of circular entry flows of fluid M1 using an integral constitutive equation, J. Non-Newt. Fluid Mech., 42 (1992), 301-314.

[69] X. L. Luo, R. I. Tanner, A pseudo-time integral method for non-isothermal viscolastic flows and its application to extrusion simulation, Rheol. Acta, 26 (1987), 499-507. 
APÊNDICE

$\mathcal{A}$

\section{Solução analítica do escoamento em canal para}

\section{um fluido KBKZ com 1-módulo de relaxamento}

Considera-se um escoamento desenvolvido em um canal com diâmetro $D / L=1$ de um fluido KBKZ com 1-módulo de relaxamento. A origem do sistema de coordenadas é localizada na entrada inferior do canal (ver Fig. A.1).

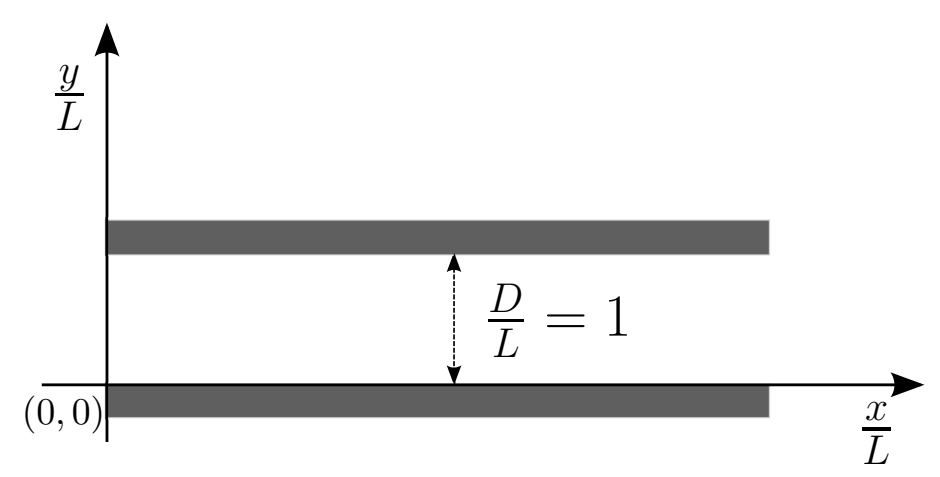

Figura A.1: Definição do canal utilizado no cálculo da solução analítica.

Procura-se soluções para as velocidades, pressão, e tensores da forma

$$
u=u(y), v(y)=0, p=p(x, y), \tau^{x y}=\tau^{x y}(y), \tau^{x x}=\tau^{x x}(y), \tau^{y y}=\tau^{y y}(y),
$$


para $y \in[0,1]$.

As componentes do tensor de Finger são definidas para escoamentos totalmente cisalhantes (ver equação 4.1), que resulta nos seguintes invariantes $I_{1}$ and $I_{2}$ necessários para o cálculo da função de decaimento $H\left(I_{1}, I_{2}\right)$ definida na equação de Papanastasiou (2.6)

$$
I_{1}=I_{2}=3+\dot{\gamma}^{2}\left(t-t^{\prime}\right)^{2}
$$

em que $\dot{\gamma}=\frac{\mathrm{d} u}{\mathrm{~d} y}$.

Nessas condições, a equação de conservação de quantidade de movimento reduz-se então à

$$
\begin{aligned}
-\frac{\partial p}{\partial x}+\frac{\partial \tau^{x y}}{\partial y} & =0 \\
-\frac{\partial p}{\partial y}+\frac{\partial \tau^{y y}}{\partial y} & =0
\end{aligned}
$$

e as componentes do tensor de tensões extra são definidas como

$$
\begin{aligned}
\tau^{x y} & =\frac{a_{1} \alpha}{W i} \int_{-\infty}^{t} e^{-\left(t-t^{\prime}\right) / W i} \frac{\dot{\gamma}\left(t-t^{\prime}\right)}{\alpha+\dot{\gamma}^{2}\left(t-t^{\prime}\right)^{2}} d t^{\prime} \\
\tau^{x x} & =\frac{a_{1} \alpha}{W i} \int_{-\infty}^{t} e^{-\left(t-t^{\prime}\right) / W i} \frac{\left[1+\dot{\gamma}^{2}\left(t-t^{\prime}\right)^{2}\right]}{\alpha+\dot{\gamma}^{2}\left(t-t^{\prime}\right)^{2}} d t^{\prime} \\
\tau^{y y} & =\frac{a_{1} \alpha}{W i} \int_{-\infty}^{t} e^{-\left(t-t^{\prime}\right) / W i} \frac{1}{\alpha+\dot{\gamma}^{2}\left(t-t^{\prime}\right)^{2}} d t^{\prime}
\end{aligned}
$$

A equação (A.4) mostra que

$$
p(x, y)=\tau^{y y}(y)+F(x)
$$

e nesse caso, a equação (A.3) pode ser escrita como

$$
\frac{\partial \tau^{x y}}{\partial y}=F^{\prime}(x)
$$

e pode-se argumentar que o lado esquerdo da equação (A.9) é função apenas de $y$. Consequentemente, $F^{\prime}$ deve ser uma constante que denota-se por

$$
C=\frac{\partial p}{\partial x}
$$


Assim,

$$
\tau^{x y}(y)=C y+h(x)
$$

e notando que $\tau^{x y}(1 / 2)=0$, por simetria, tem-se $h(x)=-\frac{1}{2} C$ e consequentemente

$$
\tau^{x y}(y)=(y-1 / 2) C
$$

Logo, a equação (A.5) pode ser escrita como

$$
(y-1 / 2) C=\frac{a_{1} \alpha}{W i} \int_{-\infty}^{t} e^{-\left(t-t^{\prime}\right) / W i} \frac{\dot{\gamma}\left(t-t^{\prime}\right)}{\alpha+\dot{\gamma}^{2}\left(t-t^{\prime}\right)^{2}} d t^{\prime}
$$

Fazendo a mudança de variáveis $s=t-t^{\prime}$, obtém-se

$$
(y-1 / 2) C=\frac{a_{1} \alpha}{W i} \int_{0}^{\infty} e^{-s / W i} \frac{\dot{\gamma} s}{\alpha+\dot{\gamma}^{2} s^{2}} d s
$$

Agora, definindo $s=\left(\alpha^{1 / 2} / \dot{\gamma}\right) \mathcal{T}$ segue que

$$
(y-1 / 2) C=K \int_{0}^{\infty} e^{-\phi \mathcal{T}} \frac{\mathcal{T}}{1+\mathcal{T}^{2}} d \mathcal{T}
$$

onde

$$
\phi=\frac{\alpha^{1 / 2}}{\dot{\gamma} W i}, \quad K=\frac{a_{1} \alpha}{\dot{\gamma} W i} .
$$

A integral na equação (A.15) pode ser resolvida, por exemplo, pelo software Mathematica, que fornece

$$
\begin{aligned}
\int_{0}^{\mathcal{T}_{\infty}} e^{-\phi \mathcal{T}} \frac{\mathcal{T}}{1+\mathcal{T}^{2}} d \mathcal{T}=\frac{1}{2} e^{-i \phi} & \left(-\operatorname{Ei}[i \phi]+\operatorname{Ei}\left[i \phi-\frac{\mathcal{T}_{\infty}}{W i}\right]\right. \\
& \left.+e^{2 i \phi}\left(-\operatorname{Ei}[-i \phi]+\operatorname{Ei}\left[-i \phi-\frac{\mathcal{T}_{\infty}}{W i}\right]\right)\right)
\end{aligned}
$$

onde $i=\sqrt{-1}$, e $\operatorname{Ei}(z)$ denota a integral exponencial dada por

$$
\operatorname{Ei}(z)=\int_{1}^{\infty} s^{-1} e^{-s z} d s
$$

Nesse caso, $\mathcal{T}_{\infty}$ é definido suficientemente grande, para ser assumido como infinito. Nota-se 
que $\operatorname{Ei}(i \phi)$ é o complexo conjugado de $\operatorname{Ei}(-i \phi)$, assim o resultado em (A.17) será real, o que é um valor esperado (ver A.14). Essa integral pode ser resolvida por um método de quadratura de alta ordem.

Tomando as condições de contorno na entrada do canal, onde a velocidade $u^{i n}(y)$ é definida por

$$
u^{i n}(y)=1-4\left(y-\frac{1}{2}\right)^{2}, \quad 0 \leq y \leq 1
$$

obtemos

$$
\int_{0}^{1} u^{i n}(y) d y=\frac{2}{3},
$$

e por conservação de massa, a solução $u(y)$, deve também satisfazer

$$
\int_{0}^{1} u(y) d y=\frac{2}{3} .
$$

Integrando por partes a equação (A.21) obtém-se

$$
\int_{0}^{1} y \dot{\gamma}(y) d y+\frac{2}{3}=0
$$

Para obter as soluções $u(y), p(x, y), \tau^{x y}(y),, \tau^{x x}(y)$ e $\tau^{y y}(y)$, primeiramente, calcula-se $\dot{\gamma}$ e $C$ pelo seguinte procedimento:

P1 - Estime um valor para $C$; que pode ser tomado inicialmente como sendo o correspondente valor Newtoniano;

P2 - Discretize o intervalo $\left(\frac{1}{2}, 1\right]$, correspondente à metade do canal, em $N$ pontos, $y j=$ $\frac{1}{2}+j * \frac{1}{2 N}, j=1,2, \cdots, N$

P3 - Calcule o perfil em escoamento totalmente cisalhante, $\dot{\gamma}_{j}$, encontrando os zeros da função (A.14), $j=1,2, \cdots, N$;

P4 - Verifique se $\dot{\gamma}_{j}$ satisfaz a equação (A.22);

P5 - Não sendo satisfeita a equação (A.22), forneça outro valor para $C$ e refaça os passos P3 e $\mathbf{P} 4$. 
Após obter os valores para $C$ e $\dot{\gamma}\left(y_{j}\right)$ usando os passos P1 - P5, o valor de $\tau^{x y}\left(y_{j}\right)$ é obtido pela equação (A.12). Os valores de $\tau^{x x}\left(y_{j}\right)$ e $\tau^{y y}\left(y_{j}\right)$ podem ser obtidos pelas equações (A.6) e (A.7), assumindo a mudança de variáveis $s=t-t^{\prime}$

$$
\begin{aligned}
\tau^{x x}\left(y_{j}\right) & =\frac{a_{1} \alpha}{W i} \int_{0}^{\infty} e^{-s / W i} \frac{1+\dot{\gamma}\left(y_{j}\right)^{2} s^{2}}{\alpha+\dot{\gamma}\left(y_{j}\right)^{2} s^{2}} d s \\
\tau^{y y}\left(y_{j}\right) & =\frac{a_{1} \alpha}{W i} \int_{0}^{\infty} e^{-s / W i} \frac{1}{\alpha+\dot{\gamma}\left(y_{j}\right)^{2} s^{2}} d s
\end{aligned}
$$

e tomando $s=\alpha^{1 / 2} \mathcal{T} / \dot{\gamma}\left(y_{j}\right)$, essas integrais se simplificam como

$$
\begin{aligned}
\tau^{x x}\left(y_{j}\right) & =K \int_{0}^{\infty} e^{-\phi \mathcal{T}} \frac{1+\alpha \mathcal{T}^{2}}{1+\mathcal{T}^{2}} d \mathcal{T}, \\
\tau^{y y}\left(y_{j}\right) & =K \int_{0}^{\infty} e^{-\phi \mathcal{T}} \frac{1}{1+\mathcal{T}^{2}} d \mathcal{T},
\end{aligned}
$$

onde $K$ e $\phi$ são dados pela equação (A.16). As integrais em (A.25) e (A.26) podem ser resolvidas pelo Mathematica, que resulta em

$$
\begin{array}{r}
\int_{0}^{\mathcal{T}_{\infty}} e^{-\phi \mathcal{T}} \frac{1+\alpha \mathcal{T}^{2}}{1+\mathcal{T}^{2}} d \mathcal{T}=-\frac{1}{2} e^{-i \phi-\frac{\mathcal{T}_{\infty}}{W i}}\left(-\frac{2 e^{i \phi}\left(-1+e^{\frac{\mathcal{T}_{\infty}}{\mathrm{Wi}}}\right) \alpha}{\phi}+i e^{\frac{\mathcal{T}_{\infty}}{\mathrm{Wi}}}(-1+\alpha)\right. \\
\left.\left(\operatorname{Ei}[i \phi]-\operatorname{Ei}\left[i \phi-\frac{\mathcal{T}_{\infty}}{W i}\right]+e^{2 i \phi}\left(-\operatorname{Ei}[-i \phi]+\operatorname{Ei}\left[-i \phi-\frac{\mathcal{T}_{\infty}}{W i}\right]\right)\right)\right) \\
\int_{0}^{\mathcal{T}_{\infty}} e^{-\phi \mathcal{T}} \frac{1}{1+\mathcal{T}^{2}} d \mathcal{T}=-\frac{1}{2} i e^{-i \phi}\left(\operatorname{Ei}[i \phi]-\operatorname{Ei}\left[i \phi-\frac{\mathcal{T}_{\infty}}{W i}\right]\right. \\
\left.+e^{2 i \phi}\left(-\operatorname{Ei}[-i \phi]+\operatorname{Ei}\left[-i \phi-\frac{\mathcal{T}_{\infty}}{W i}\right]\right)\right)
\end{array}
$$

